\section{Mortalidad por Causas Violentas en Adolescentes y Jóvenes: Un Desafio para la Región de las Américas}

\section{Mortality from violent causes in adolescents and young people: a challenge for the Region of the Americas}

João Yunes (*)

Departamento de Saúde Materno-Infantil

Faculdade de Saúde Pública

Universidade de São Paulo

Av. Dr. Arnaldo, 715

01246-904 São Paulo, SP - Brasil

Tamara Zubarew (**)

Facultad de Medicina

Pontificia Universidade Catolica de Chile

Santiago Chile

(*) Profesor Titular del Departamento de Salud Materno Infantil de la Faculdad de Salud Publica de la Universidad de São Paulo-Brasil.

(**) Profesor de Pediatria de la Facultad de Medicina de la Pontificia Universidad Catolica de Chile.

\section{Resumen}

Este trabajo describe la tendencia de la mortalidad por homicidios, suicidios, accidentes de tráfico y otras causas externas, en la población total, adolescente y joven, de 16 países de la Región de las Américas. Se utiliza información del período comprendido entre los años 1980 y para el ultimo año disponible de la decada de 1990, proveniente del Banco de Datos de la Organización Panamericana de la Salud. Se realiza una descripción diferencial de los subgrupos de adolescentes y jóvenes, por edad y sexo. Los resultados demuestran que en la mayor parte de los países (Canadá, EEUU, Ecuador, México, Chile, Costa Rica, Trinidad \& Tobago y El Salvador) hay una tendencia decreciente en la mortalidad por causas externas, tanto en la población total como en adolescentes y jóvenes. Colombia y Brasil son los únicos países analizados que presentan tasas de mortalidad por causas externas francamente ascendentes en los grupos estudiados. Los países con mayores tasas de mortalidad por causas externa en adolescentes y jóvenes, en orden decreciente, son: Colombia, El Salvador, Venezuela, Brasil y Puerto Rico. Los accidentes de tráfico son el determinante principal de la mortalidad por causas externas en la población total como en adolescentes y jóvenes, presentando tendencias decrecientes en casi todos los países estudiados. En 10 países se observa un ascenso progresivo de la mortalidad por homicidio en todos los grupos estudiados (Colombia, Puerto Rico, Trinidad \& Tobago, Argentina, Uruguay, Panamá, EEUU, Venezuela, Ecuador y Brasil). Los países con mayores tasa de mortalidad por homicidio entre los varones de 15 a 19 años son, en orden decreciente: Colombia, El Salvador, Puerto Rico, Venezuela y Brasil. Los varones de 20 a 24 años presentan las tasas mas altas de homicidio dentro de los grupos estudiados. Hay un aumento alarmante de la tasa de homicidio en el grupo de varones de 15 a 19 años, especialmente en EEUU y Brasil. La mortalidad por suicidio es un problema creciente entre adolescentes y jóvenes de Cuba, Canadá, EEUU, Trinidad \& Tobago, Argentina y El Salvador. Las tasas crecientes de mortalidad por homicidio reflejan la necesidad inminente de programas de vigilancia epidemiológica y de prevención de violencia dirigidos a adolescentes y jóvenes de la Región de las Américas.

Palabras claves: Adolescencia. Jovenes. Tasas de mortalidad. Causas violentas. Homicidio. Suicidio. Accidentes de tráfico. Causas externas. 
This paper describes mortality trends for homicides, suicides, traffic accidents and other external causes in the entire population, adolescents, and youngsters, from 16 countries in the Region of the Americas. This study used the information compiled by the Pan American Health Organization data bank from 1980 until the last year in the 90's in which data was available for each country. A description of different subgroups of adolescents and young people, by age, and sex, is provided. Results indicate that in most countries (Canada, USA, Ecuador, Mexico, Chile, Costa Rica, Trinidad and Tobago, and El Salvador), mortality trends due to external causes have been decreasing in the entire population, as well as among adolescents and young people. Colombia and Brazil are the only countries analyzed which have increasing mortality rates by external causes in their populations. The countries with highest mortality rates due to external causes among adolescents and young people, in decreasing order, are: Colombia, El Salvador, Venezuela, Brazil and Puerto Rico. The primary external causes of death in the entire population, as well as among adolescents and young people, are traffic accidents. However, in almost all of the countries analyzed, death rates are decreasing. Ten of the countries analyzed revealed a progressive increase in homicides in all of the age groups studied (Colombia, Puerto Rico, Trinidad and Tobago, Argentina, Uruguay, Panama, USA, Venezuela, and Brazil). The countries with the highest homicide rates among young men, between 15 and 19 years of age, in decreasing order, are: Colombia, El Salvador, Puerto Rico, Venezuela and Brazil. Young men, between 20 and 24 years of age, were the subgroup with the highest homicide rates. In the United States and Brazil, there is an alarming increase in the homicide rate of boys between 15 and 19 years of age. Suicide is a growing problem among adolescents and young people in Cuba, Canada, USA, Trinidad and Tobago, Argentina, and El Salvador. Increasing mortality rates due to homicide reflect the pressing need for epidemiological surveillance and violence prevention programs directed towards adolescents and young people in the Region of the Americas.

Keywords: Adolescence. Young people. Mortality rates. Violence causes. Homicide. Suicide. Traffic accidents. External causes.

\section{Introduccion}

Durante los últimos decenios ha habido un aumento considerable en el nivel de violencia y todas sus manifestaciones en todo el mundo. La violencia ha adquirido carácter epidémico y se ha convertido en uno de los problemas de Salud Pública mas serios de la Región de las Américas. Respecto a América Latina y el Caribe, en 1995 la tasa de mortalidad por causas externas en el total de la poblaccíon sufre una variación de 46,9 en Costa Rica hasta 107,8 en Colombia. La tasa de mortalidad por causas externas (homicidios, suicidios, accidentes de tráfico y otras lesiones accidentales) ha comenzado a afectar significativamente la tasa de mortalidad general ${ }^{1}$.

La situación es particularmente alarmante en el grupo de adolescentes y jóvenes (10 a 24 años) ${ }^{2}$. De acuerdo a la Organización Mundial de la Salud, se define adolescencia al período comprendido entre 10 y 19 años y juventud entre 15 y 24 años. La población adolescente representa un $20,3 \%$ de la población total de la Región de las Américas. El 31,6\% de esta población se encuentra entre los 10 y 24 años de edad ${ }^{3}$.

Del total de homicidios registrados en la Región de las Américas en 1990, el 28.7\% corresponde a jóvenes entre 15 y 24 años. En 10 de los 21 países de la Región, con más de un millón de habitantes, el homicidio es la segunda causa de muerte en el grupo de 15 a 24 años, y está entre las cinco primeras causas en 17 de los 21 países ${ }^{4}$.

La muerte prematura por violencia de los jóvenes tiene como consecuencia altos costos económicos y sociales. La concentración de los homicidios y de otras causas externas de mortalidad en los adolescentes y jóvenes determina que la violencia sea la principal causa de años de vida perdidos en la mayor parte de los países de la Región, produciendo una pérdida promedio de 30 a 40 años de vida por defunción ${ }^{3}$. El costo económico directo de las muertes e incapacidades físicas, como resultado de actos de violencia, está estimado en 10.000 millones de dólares al año. Esto representa 
alrededor del 20\% del total del gasto nacional en salud de los países de la Región ${ }^{2}$.

Las muertes por violencia constituyen sólo una pequeña proporción del problema. De acuerdo con estimaciones de la Organización Mundial de la Salud, por cada niño y adolescente que muere de una lesión traumática, hay 15 que quedan severamente afectados y otros 30 a 40 que registran daños que requieren de cuidados médicos, psicológicos o de rehabilitación. No existe registro sistemático en América Latina de las formas no letales de violencia. En la mayor parte de los países de la región no se han implementado sistemas de vigilancia epidemiológica de hechos violentos. Sin embargo, resultados de investigaciones aisladas permiten apreciar el espectro y la frecuencia significativamente mayor de estas lesiones que, aunque no matan, determinan las condiciones y la calidad de vida futura de adolescentes y jóvenes.

La adolescencia es uno de los grupos de edad más afectados por la violencia en sus diferentes formas. La violencia durante la adolescencia no sólo se manifiesta como un daño físico sino también incluye abuso sexual, negligencia, abuso emocional, ataque verbal, amenazas, acoso sexual y otras formas de abuso psicológico.

Los adolescentes no son sólo víctimas, son también actores y/o agentes originales o intermediarios de variadas formas de violencia. Los actos de violencia afectan a la víctima directa y también a sus miembros familiares, compañeros de estudio o trabajo, proveedores de servicios y a toda la comunidad. Todas las formas de violencia tienen efectos dañinos a corto y largo plazo en la salud física y mental, limitando la funcionalidad individual y social. Además son responsables de enormes costos económicos a la sociedad, generan gastos en salud y disminuyen la productividad de las comunidades. La violencia en adolescentes y jóvenes es evitable y prevenible ${ }^{5}$. Características individuales, experiencias familiares, relaciones de pares, acceso a armas, consumo de alcohol y otras drogas, exposición a violencia en los medios de comuni- cación y otros factores políticos, culturales y sociales se interrelacionan y juegan un papel fundamental en la ocurrencia de la violencia en jóvenes.

Los adolescentes y jóvenes de la Región de las Américas están expuestos a cantidades crecientes de violencia a través de los medios de comunicación. Actualmente existe consenso en que esta exposición es una de las causas de conducta agresiva, crímenes y violencia en jóvenes. A pesar que no constituyen la causa única de la violencia, la influencia de los medios de comunicación es el factor contribuyente más factible de revertir ${ }^{1,6}$.

Dada su naturaleza multifactorial, la violencia se debe prevenir mediante la participación coordinada y activa de todos los sectores de la sociedad, con un enfoque multidisciplinario e intersectorial.

Para la Organización Panamericana de la Salud (OPS) el tema de la salud de los adolescentes y jóvenes ha sido de fundamental importancia, tanto por sus efectos sociales, económicos y sus implicaciones sobre los servicios de salud y la promoción de salud en este grupo etáreo. En la XXXVI Reunión del Consejo Directivo de la OPS, los países miembros aprobaron el plan de acción sobre salud de adolescentes en las Américas. El Consejo Directivo emitió la Resolución CD 36/18 mediante la cual se instó a los gobiernos a establecer políticas y planes nacionales de salud integral de adolescentes, a desarrollar vínculos de colaboración entre las entidades responsables de la salud de los adolescentes, a promover la participación de los adolescentes en la promoción de salud y a colaborar en programas específicos. También la OPS se ha preocupado reiteradamente del fenómeno de la violencia en grupos determinados. Ha impulsado la recolección y sistematización de información epidemiológica sobre la materia y publicado documentos sobre el tema. En Noviembre de 1994, durante la XXXVII Reunión del Consejo Directivo de la OPS, los países miembros emitieron un documento en el que consideran las conductas violentas como un problema de Salud 
Pública de gran magnitud y relevancia en la región de las Américas y estiman que el sector salud debe contribuir a la búsqueda de soluciones y a la aplicación de medidas preventivas y de control de todas las formas de violencia. El Consejo Directivo aprobó en esa oportunidad el Plan de Acción Regional sobre Violencia y Salud, en el que se identifican objetivos y se proponen estrategias y actividades para la prevención de la violencia en la región, en coordinación con otros sectores sociales, públicos y privados. Las orientaciones que promueven el Plan de Acción Regional responden a los principios de: integralidad, equidad, compromiso político, cultura ciudadana, conocimiento en función de la acción y participación social.

A pesar que las muertes violentas provocan la pérdida de una gran cantidad de vidas jóvenes, limitan la funcionalidad individual y social e impactan negativamente la economía, la implementación de sistemas de vigilancia epidemiológica y de programas de prevención de violencia en adolescencia y juventud es escasa en los países de la región. Los programas actualmente existentes son parciales y fragmentados, carecen de enfoque integral e intersectorial y carecen de evaluación.

Existen escasos estudios publicados acerca de la magnitud de la violencia en los países de las Américas. La comparación de la situación de la violencia entre países es muy difícil dada la ausencia de definiciones comunes y de sistemas de vigilancia epidemiológica de hechos violentos en los países de la Región. En 1993, Yunes publicó el artículo "Mortalidad por causas violentas en la Región de las Américas”, donde analiza la situación de la población general y, en particular, la población menor de 24 años. En 1994, Yunes y Rajs ${ }^{8}$ publicaron el trabajo "Tendencias de la Mortalidad por Causas Violentas en la Población General y entre los Jóvenes de la Región de las Américas“, donde se analiza con mayor detalle la mortalidad por causas externas de los grupos de adolescentes y jóvenes de 15 países, entre los años 1979 y 1980.
El objetivo del presente trabajo es describir la tendencia de la mortalidad por causas externas o violentas en la Región de las Américas en la población total y adolescente y joven (10 a 24 años), entre los años 1980 y 1997, con análisis diferencial de subgrupos de adolescentes por edad y sexo; con el fin de contar con un documento que facilite la implementación de políticas y programas acordes a las necesidades de los diferentes países de la región.

Para los fines de este estudio se entiende como causa externa o violenta de defunción a toda causa incluida en la "Clasificación Suplementaria de Traumatismos y Envenenamientos" de la Novena Revisión de la Clasificación Internacional de Enfermedades, Traumatismos y Causas de Defunción (CIE-9).

\section{Material y Metodo}

Los datos demográficos y tasas de mortalidad fueron obtenidos del Banco de Datos de la Organización Panamericana de la Salud-OPS, confeccionado con base en informes anuales de mortalidad remitidos por los países de la región. Las causas de muerte han sido agrupadas de acuerdo a la Clasificación Internacional de Enfermedades, Novena Revisión (CIE-9). En cada grupo de edad y sexo se analizan las siguientes causas de muerte:

- Tasa de Mortalidad por Todas las Causas Externas (Códigos E800-E999);

- Tasa de Mortalidad por Accidentes de Tráfico de Vehículo de Motor (Códigos E810-E819);

- Tasa de Mortalidad por Suicidios y Lesiones Autoinflingidas (Códigos E950E959);

- Tasa de Mortalidad por Homicidios y Lesiones Intencionales y Lesiones Resultantes de Operaciones de Guerra o por Intervención Legal (Códigos E960E969;E970-E978; E990-E999).

Además se analizaron las tasas de mortalidad de la población total utilizando las mismas categorías, como un patrón de 
comparación para establecer el peso relativo de la mortalidad por cada causa en el grupo de adolescentes y jóvenes.

\section{Ambito geográfico y período de estudio}

Los factores que determinaron la inclusión de los 16 países analizados en el estudio fueron: existencia de información actualizada en el Banco de Datos de la OPS; tamaño de la población; adecuada calidad de estadísticas vitales (medida por la proporción de muertes externas atribuidas a lesiones que se ignora si fueron accidentales o intencionalmente infligidas); información disponible para la totalidad del período analizado e información disponible para subgrupos de causas externas.

Los países que cumplieron con los criterios de inclusión fueron:

- América Central: Costa Rica, El Salvador y Panamá

- Norte América: Estados Unidos, Canadá y México

- América Latina: Area Andina: Colombia, Ecuador y Venezuela

- Cono Sur: Chile, Argentina, Uruguay y Brasil

- Caribe Latino: Cuba y Puerto Rico

- Caribe Inglés: Trinidad y Tobago

\section{Distribución por edad y sexo}

Se analizaron las tasas de mortalidad específica por edad y sexo, en los siguientes grupos etáreos: Todas las edades, 10 a 14, 15 a 19, 20 a 24,10 a 19 y 15 a 24 años.

\section{Calidad de la información}

La calidad del registro de defunciones varía considerablemente entre los países, en forma cuantitativa (subregistro de muertes) y cualitativa (calidad de certificación de la causa de muerte). Este estudio se realizó en base a las defunciones registradas. Aunque en varios países hay subregistro de defunciones, en el caso de las defunciones por causas externas el subregistro es menor debido a disposiciones legales. Los países con mayores subregistros, como Perú, República Dominicana, Paraguay, Nicaragua y Honduras, no fueron incluidos en el estudio. Cabe destacar que, de los países incluidos, Ecuador, Panamá, Brasil y El Salvador presentan un subregistro de alrededor de un $20 \%$, que debe ser considerado en el análisis. Algunos países no cuentan con la información detallada para los grupos de edad escogidos en este estudio, otros no informan grupos detallados de causa externa (Cuba, Honduras), otros con altos porcentajes de defunciones por edad y/o por sexo ignorados, otros con varios años sin información o información parcial y, por último, algunos países cuya distribución de causas de muerte sugiere importantes deficiencias de la calidad de la certificación de la muerte (como en Bahamas, Chile y Guatemala), donde la proporción de defunciones externas atribuidas a "Lesiones en que se ignora si fueron Accidental o Intencionalmente Inflijidas" es superior al $20 \%$, o en que la citada proporción es nula, como en El Salvador. A diferencia de lo que ocurre con otras causas de muerte, las defunciones por causas externas también se ven influidas por factores de orden jurídico-político y legal. Otra fuente de error en el cálculo de las tasas de mortalidad es la estimación del denominador, la población de cada grupo etáreo de cada país.

\section{Resultados}

Se presenta las tasas de mortalidad por todas las causas tanto para la población total como para la población masculina y femenina, permietindo establecer comparación del peso relativo de la mortalidad por cada causa en el grupo de adolescentes y jóvenes (Cuadros 1 a 1.2, en Anexo).

\section{A. Países de América Central}

\section{PANAMA}

La tendencia de la mortalidad por causas externas de la población total de Pana- 
má es ligeramente descendente entre los años 1980 y 1988 (10\% de descenso) para sufrir posteriormente un aumento de un $30 \%$ en los hombres entre los años 1988 y 1989, coincidente con el conflicto bélico externo sufrido por el país. Entre los años extremos estudiados (1980-1987) hay un aumento de un $7 \%$ en la población total (Cuadros 1.3 a 1.4, en Anexo).

La tendencia en el grupo de hombres de 10 a 14 y 15 a 19 años es levemente descendente entre los años 1980 y 1989. En cambio, en el grupo de 20 a 24 años hay una tendencia descendente hasta el año 1988, seguido por un aumento de mortalidad por causas externas de un $44 \%$ entre los años 1988 y 1989. A un cuando entre los hombrens de 20 a 24 anos se observa una reducción de la tasa de mortalidad por causas externas en $1996(121,7)$ esta vuelve a aumentar en 1997 (136,4), o sea, $12 \%$ de aumento en esse período. Ese aumento es más significativo entre los hombres de 15 a 19 años, entre los cuales alcanza 21\% (Cuadro 1.a) .

La mortalidad por causas externas en las mujeres de la población total y en los adolescentes y jóvenes no sufre cambios significativos entre los años extremos del período estudiado (Cuadros 1.5 en Anexo y 1.a).

La causa externa mas frecuente de mortalidad en Panamá son los accidentes de tráfico, tanto en la población total como en adolescentes, excepto durante el año 1989, donde el homicidio ocupa el primer lugar en la población de varones de todas las edades y varones entre 20 y 24 años. La mortalidad por accidentes de tráfico ha disminuido en un $24 \%$ en la población total y en un $39 \%$ en el grupo de jóvenes varones de 20 a 24 años entre los años 1980 y 1989. Pero esa tendencia se invierte, superando en $1997(50,4)$ todos los valores referentes a los años de la decada de ochenta. De 1996 para 1997 la tasa de mortalidad por accidentes de vehiculos a motor en el grupo masculino de 20 a 24 años, apresentó un aumento de $35 \%$ y en el de 15 a 19 años alcanzó 58\% (Cuadro 1.b).

Ya en el sexo femenino, con valores significativamente menores, puedense observar, para los mismos grupos etários, au- mento en las respectivas tasas de mortalidad, entre los años de 1996 y 1997, de 69\% y $24 \%$, respectivamente (Cuadro 1.b). La relación hombre/mujer de la mortalidad por accidentes de tránsito es $4 / 1$ en el grupo de 15 a 24 años y $2 / 1$ en el grupo de 10 a 14 años (Cuadro 1.b).

Respecto a la mortalidad por suicidio, hay una tendencia al aumento en la población total $160 \%$ de incremento entre los años extremos (Cuadro 1.9, en Anexo), como también en los adolescentes y jóvenes de ambos sexos. Entre los años 1981 y 1997 se triplica la tasa de suicidios en los varones de 15 a 24 años, de 2,5 a 9,2 por 100.000 habitantes. En todas las categorías, excepto 10 a 14 años, en que el número de fallecimientos es insignificante, las tasas de mortalidad por suicidio son 3 a 4 veces mayores en hombres que en mujeres (Cuadro 1.c).

La tasa de mortalidad por homicidios en la población total casi se cuadruplica entre los años 1980 y 1988, elevándose bruscamente en un $103 \%$ entre 1988 y 1989 (Cuadro 1.12, en Anexo), año del conflicto bélico. Entre los adolescentes y jóvenes varones hay un progresivo y dramático aumento de la mortalidad por homicidio en el período estudiado (Cuadro 1.d). En el grupo de hombres de 15 a 19 años las tasas se cuadruplican entre los años 1980 y 1988, ascendiendo después en un $39 \%$ hasta el año 1989. En el grupo de hombres de 20 a 24 años el aumento entre los años 1980 y 1988 es de un $413 \%$ y 103\% entre 1988 y 1989.

Los datos de 1996 y 1997 confirman el aumento de muertes por homicidios para ambos los sexos en los mismos grupos de edades. Entre los hombres de 15 a 19 años fue de $26 \%$ y de $24 \%$ entre los de 20 a 24 años, sin considerar como causa presumida el conflicto bélico, tal como en los años finales de la decada de ochenta (Cuadro 1.d).También hay aumento de las tasas de homicidio entre mujeres de 15 a 24 años, significativamente menor que en los hombres.

En resumen, la mortalidad por causas externas en adolescentes y jóvenes en Panamá se ha mantenido relativamente estable como producto de una disminución de 
Cuadro 1.a - Tasa* de mortalidad de adolescentes y jóvenes por causas externas, según edad y sexo - Panamá 1980-1997

Table 1.a - Mortality rate among adolescents and young adults due to external causes, by age and sex-Panamá 1980-1997

\begin{tabular}{|c|c|c|c|c|c|c|c|c|c|c|}
\hline \multirow{2}{*}{$\begin{array}{l}\text { Edad } \\
\text { Años }\end{array}$} & \multicolumn{2}{|c|}{10 a 14 años } & \multicolumn{2}{|c|}{15 a 19 años } & \multicolumn{2}{|c|}{20 a 24 años } & \multicolumn{2}{|c|}{10 a 19 años } & \multicolumn{2}{|c|}{15 a 24 años } \\
\hline & M & $\mathrm{F}$ & M & $\mathrm{F}$ & M & $\mathrm{F}$ & M & $\mathrm{F}$ & M & $\mathrm{F}$ \\
\hline 1980 & 22,0 & 7,4 & 80,7 & 15,9 & 123,3 & 28,1 & 49,2 & 11,4 & 100,0 & 21,4 \\
\hline 1981 & 25,6 & 8,1 & 60,7 & 17,3 & 125,8 & 20,4 & 41,9 & 12,4 & 90,2 & 18,7 \\
\hline 1982 & 26,0 & 10,3 & 58,3 & 21,2 & 116,5 & 13,5 & 41,1 & 15,5 & 84,9 & 17,7 \\
\hline 1983 & 26,5 & 9,4 & 54,2 & 17,4 & 110,0 & 26,3 & 39,6 & 13,2 & 79,8 & 21,5 \\
\hline 1984 & 22,6 & 12,4 & 52,1 & 17,8 & 101,9 & 17,6 & 36,6 & 15,0 & 73,7 & 17,6 \\
\hline 1985 & 17,9 & 11,5 & 60,2 & 14,2 & 118,9 & 19,0 & 38,1 & 12,8 & 85,8 & 16,2 \\
\hline 1986 & 18,7 & 7,7 & 69,8 & 18,9 & 85,3 & 14,8 & 43,5 & 13,1 & 76,1 & 17,1 \\
\hline 1987 & 19,4 & 9,2 & 71,7 & 14,5 & 114,3 & 16,2 & 44,8 & 11,8 & 91,6 & 15,3 \\
\hline 1988 & 22,4 & 13,2 & 69,0 & 21,4 & 96,5 & 13,3 & 45,2 & 17,3 & 80,3 & 17,3 \\
\hline 1989 & 20,9 & 6,2 & 68,5 & 22,0 & 139,0 & 17,2 & 44,3 & 14,1 & 100,0 & 19,4 \\
\hline 1996 & 20,4 & 9,5 & 59,8 & 18,0 & 121,7 & 17,5 & 39,4 & 13,6 & 90,4 & 17,7 \\
\hline 1997 & 18,1 & 5,8 & 72,2 & 18,6 & 136,4 & 16,7 & 44,0 & 11,9 & 103,8 & 17,6 \\
\hline
\end{tabular}

Fuente/Source:OPS. Sistema de Información Técnica

*Tasa/Rate x 100.000 habitantes

M:Masculino/Male

F:Femenino/Female

Cuadro 1.b - Tasa* de mortalidad de adolescentes y jóvenes por accidentes de vehículo a motor, según edad y sexo - Panamá 1980-1997

Table 1.b - Mortality rate among adolescents and young adults due to traffic accidents, by age and sex-Panamá 1980-1997

\begin{tabular}{ccccccccccc}
\hline Edad & \multicolumn{2}{c}{ 10 a 14 años } & \multicolumn{2}{c}{ 15 a 19 años } & \multicolumn{2}{c}{20 a 24 años } & \multicolumn{2}{c}{10 a 19 años } & \multicolumn{2}{c}{15 a 24 años } \\
\hline Años & M & F & M & \multicolumn{1}{c}{ F } & M & F & M & F & M & F \\
\hline 1980 & 2,4 & 2,5 & 27,5 & 6,5 & 43,3 & 13,5 & 14,0 & 4,4 & 34,7 & 9,7 \\
1981 & 8,5 & 3,2 & 20,5 & 8,2 & 41,9 & 8,6 & 14,1 & 5,6 & 30,2 & 8,4 \\
1982 & 12,2 & 6,3 & 17,4 & 8,8 & 30,9 & 4,2 & 14,6 & 7,5 & 23,6 & 6,7 \\
1983 & 8,3 & 3,1 & 19,5 & 10,4 & 49,0 & 7,1 & 13,6 & 6,6 & 33,0 & 8,9 \\
1984 & 7,5 & 4,7 & 15,7 & 5,1 & 36,9 & 4,9 & 11,4 & 4,9 & 25,0 & 5,0 \\
1985 & 6,7 & 3,8 & 13,0 & 8,3 & 31,1 & 2,9 & 9.7 & 6,0 & 21,0 & 5,7 \\
1986 & 6,0 & 0,8 & 19,0 & 4,9 & 32,1 & 5,6 & 12,3 & 2,8 & 24,8 & 5,3 \\
1987 & 6,0 & 3,1 & 24,4 & 7,3 & 35,7 & 5,4 & 14,9 & 5,1 & 29,7 & 6,4 \\
1988 & 7,5 & 5,4 & 18,6 & 8,7 & 29,6 & 4,4 & 12,9 & 7,1 & 23,3 & 6,6 \\
1989 & 5,2 & 1,6 & 23,8 & 7,9 & 26,3 & 4,3 & 14,4 & 4,7 & 24,5 & 6,1 \\
1996 & 7,7 & 5,8 & 11,4 & 6,3 & 37,2 & 5,6 & 9,5 & 6,0 & 24,1 & 5,9 \\
1997 & 5,6 & 2,2 & 18,0 & 7,8 & 50,4 & 9,5 & 11,6 & 4,9 & 34,0 & 8,6 \\
\hline
\end{tabular}

Fuente/Source:OPS. Sistema de Información Técnica

*Tasa/Rate x 100.000 habitantes

M:Masculino/Male

F:Femenino/Female

las tasas de mortalidad por accidentes de tráfico y un aumento de las tasas de mortalidad por homicidio y suicidio en el período comprendido entre 1980 y 1997 . El brusco aumento de muertes de causa externa entre 1988 y 1989 en el grupo de jóvenes de 20 a 24 años se explica por el aumento de las muertes por homicidio y otras lesiones intencionales debido al conflicto bélico sufrido. 
Cuadro 1.c - Tasa* de mortalidad de adolescentes y jóvenes por suicidio, según edad y sexo Panamá 1980-1997

Table 1.c - Mortality rate among adolescents and young adults due to suicide, by age and sex Panamá 1980-1997

\begin{tabular}{cccccccccccc}
\hline Edad & \multicolumn{2}{c}{10 a 14 años } & \multicolumn{2}{c}{15 a 19 años } & \multicolumn{2}{c}{20 a 24 años } & \multicolumn{2}{c}{10 a 19 años } & \multicolumn{3}{c}{15 a 24 años } \\
\hline Años & M & F & M & F & M & F & M & F & M & F \\
\hline 1980 & 1,6 & 0,0 & 0,0 & 3,7 & 5,6 & 1,1 & 0,8 & 1,7 & 2,5 & 2,6 \\
1981 & 0,8 & 0,8 & 1,8 & 0,9 & 4,3 & 1,1 & 1,2 & 0,9 & 2,9 & 1,0 \\
1982 & 0,0 & 0,0 & 1,7 & 1,8 & 4,1 & 1,0 & 0,8 & 0,8 & 2,8 & 1,4 \\
1983 & 0,0 & 1,6 & 2,5 & 0,0 & 8,0 & 3,0 & 1,2 & 0,8 & 5,0 & 1,4 \\
1984 & 0,8 & 0,0 & 3,3 & 0,8 & 1,9 & 1,0 & 2,0 & 0,4 & 2,6 & 0,9 \\
1985 & 0,0 & 0,8 & 1,6 & 0,8 & 7,5 & 2,9 & 0,8 & 0,8 & 4,3 & 1,8 \\
1986 & 1,5 & 0,8 & 7,9 & 4,1 & 6,4 & 2,8 & 4,6 & 2,4 & 7,1 & 3,5 \\
1987 & 1,5 & 0,8 & 4,7 & 1,6 & 8,9 & 5,4 & 3,1 & 1,2 & 6,7 & 3,4 \\
1988 & 0,7 & 1,6 & 7,0 & 4,8 & 8,7 & 2,7 & 3,8 & 3,1 & 7,6 & 3,7 \\
1989 & 0,7 & 0,0 & 3,1 & 2,4 & 11,0 & 0,9 & 1,9 & 1,2 & 6,7 & 1,6 \\
1996 & 0,0 & 0,7 & 3,0 & 2,3 & 14,7 & 1,6 & 1,5 & 1,5 & 8,8 & 2,0 \\
1997 & 0,7 & 0,0 & 6,8 & 3,9 & 11,6 & 2,4 & 3,6 & 1,9 & 9,2 & 3,1 \\
\hline
\end{tabular}

Fuente/Source:OPS. Sistema de Información Técnica

*Tasa/Rate x 100.000 habitantes

M:Masculino/Male

F:Femenino/Female

Cuadro 1.d - Tasa de mortalidad de adolescentes y jóvenes por homicidio, según edad y sexo Panamá 1980-1997

Table 1.d - Mortality rate among adolescents and young adults due to homicide, by age and sex Panamá 1980-1997

\begin{tabular}{|c|c|c|c|c|c|c|c|c|c|c|}
\hline \multirow{2}{*}{$\frac{\text { Edad }}{\text { Años }}$} & \multicolumn{2}{|c|}{10 a 14 años } & \multicolumn{2}{|c|}{15 a 19 años } & \multicolumn{2}{|c|}{20 a 24 años } & \multicolumn{2}{|c|}{10 a 19 años } & \multicolumn{2}{|c|}{15 a 24 años } \\
\hline & M & $\mathrm{F}$ & M & $\mathrm{F}$ & M & $\mathrm{F}$ & M & $\mathrm{F}$ & M & $\mathrm{F}$ \\
\hline 1980 & 0,8 & 0,0 & 3,7 & 0,9 & 4,4 & 2,2 & 2,1 & 0,4 & 4,0 & 1,5 \\
\hline 1981 & 0,0 & 0,0 & 3,6 & 2,7 & 11,8 & 1,1 & 1,7 & 1,3 & 7,3 & 2,0 \\
\hline 1982 & 0,0 & 0,0 & 2,6 & 1,8 & 16,5 & 1,0 & 1,2 & 0,8 & 9,0 & 1,4 \\
\hline 1983 & 0,0 & 0,0 & 4,2 & 0,9 & 6,0 & 1,0 & 2,0 & 0,4 & 5,0 & 0,9 \\
\hline 1984 & 0,0 & 1,6 & 2,5 & 3,4 & 6,8 & 1,0 & 1,2 & 2,4 & 4,4 & 2,3 \\
\hline 1985 & 0,0 & 0,0 & 5,3 & 10,6 & 24,5 & 2,9 & 2,6 & 5,1 & 16,7 & 7,0 \\
\hline 1986 & 0,7 & 1,5 & 11,1 & 2,5 & 17,4 & 2,8 & 5,8 & 2,0 & 13,9 & 2,6 \\
\hline 1987 & 2,2 & 0,8 & 14,2 & 1,6 & 25,9 & 0,9 & 8,0 & 1,2 & 19,7 & 1,3 \\
\hline 1988 & 0,7 & 0,0 & 15,5 & 1,6 & 22,6 & 3,5 & 8,0 & 0,8 & 18,5 & 2,5 \\
\hline 1989 & 0,7 & 0,0 & 21,5 & 3,1 & 45,8 & 3,4 & 11,0 & 1,6 & 32,4 & 3,2 \\
\hline 1996 & 0,0 & 0,7 & 19,7 & 2,3 & 35,7 & 1,6 & 9,5 & 1,5 & 27,6 & 2,0 \\
\hline 1997 & 0,7 & 0,7 & 24,8 & 3,9 & 44,2 & 3,2 & 12,3 & 2,2 & 34,4 & 3,5 \\
\hline
\end{tabular}

Fuente/Source:OPS. Sistema de Información Técnica

*Tasa/Rate x 100.000 habitantes

M:Masculino/Male

F:Femenino/Female

\section{COSTA RICA}

En Costa Rica se observa una disminución paulatina de las tasas de mortalidad por causas externas de un $4,6 \%$ en la pobla- ción total en el período estudiado: 1981 1991 (Cuadros 1.3 a 1.5, en Anexo). Mayor tendencia a la disminución se observa en el grupo de adolescentes y jóvenes, con un descenso de un $36 \%$ en el grupo de hom- 
bres de 10 a 14 años, $16 \%$ entre hombres de 15 y 19 años y 33\% entre hombres de 20 a 24 años. Porcentajes semejantes de descenso se observa en la población de adolescentes mujeres, siendo las tasas de mortalidad por causas externas 2 a 3 veces menores en las mujeres de 10 a 19 años y hasta 6 veces menores en el grupo de 20 a 24 años, respecto a los respectivos grupos etáreos de hombres (Cuadro 2.a).

Es observado que esa tendencia se invierte a partir de 1992, para ambos los sexos, en los respectivos grupos etários. En 1995 se puede constatar que entre los hombres la tasa de mortalidad por causas externas son las mas altas en todo el período analisado (1981 - 1995). El mayor acrecimo verificase en el grupo etário de 20 a 24 años (66\%), considerandose los años de 1991 y 1995. Aún cuando en niveles significativamente menores, entre las mujeres también se observa que las respectivas tasas aumentaron, siendo el mayor crecimiento en el grupo etário de 20 a 24 años (23\%) en el mismo período (Cuadro 2.a).

La causa externa de mortalidad más frecuente en Costa Rica para toda la población y para el grupo de adolescentes y jóvenes corresponde a accidentes del tráfico. La tasa de mortalidad por accidentes de tráfico ha sufrido un leve aumento de un $7,2 \%$ en la población total entre los años 1981 y 1991. En cambio, ésta ha disminuido en todos los grupos de adolescentes y jóvenes, excepto en el grupo de mujeres de 15 a 19 años, en que hay un aumento en la tasa de 1,4 a 4,9 por 100.000 entre los mismos años. La tasa de mortalidad por accidentes del tráfico es mayor en los hombres en todos los grupos de edad y la diferencia se acentúa con la edad en el grupo de jóvenes (Cuadro 2.b), en especial entre los hombres del grupo etário de 20 a 24 años, cuya tasa practicamente duplicó, pasando de 18,1 em 1991, para 36,5 em 1995. Eso significa aumento considerable (102\%), en muertes por accidentes de trafico entre los jovenes.

La mortalidad por homicidio en la población total se mantiene estable durante el período estudiado, con tasas de 4,1 por
100.000 en 1991. (6,6 por 100.000 en hombres y 1,6 por 100.000 en mujeres) La tasa de mortalidad por homicidio en adolescentes y jóvenes en Costa Rica es baja y a sufrido una leve disminución entre los años 1981 y 1991 en todos los grupos de edad y sexo. En el grupo de 15 a 19 años la tasa descendió de 4,2 a 1,3 por 100.000 en el grupo de hombres y de 2,9 a 0,7 por 100.000 en el grupo de mujeres (Cuadro 2.d).

Todavia con valores menores en relación a las tasas de mortalidad por accidentes de vehículo a motor, los datos muestran que las muertes por homicidios sufreran aumentos considerables en los años noventa, alcanzando 15,4 em 1995, entre los hombres de 20 a 24 años, lo que representa un aumento de $130 \%$ en relación a 1991 (Cuadro 2.d).

La mortalidad por suicidio se mantiene estable en la población total entre los años 1981 y 1991, con una tasa de 4,2 por 100.000 en 1991. En el grupo de adolescentes de 10 a 19 años no hay cambios significativos en la mortalidad por suicidio (Cuadro 2.c). En cambio, las tasas disminuyen entre los jóvenes de 20 a 24 años; de 14,3 a 7,4 por 100.000 en los hombres y de 4,1 a 1,4 por 100.000 en las mujeres. Sin embargo, en los años noventa esa tendencia se invierte, sobre todo en el año de 1995, cuyo valor para los hombres do grupo etário de 20 a 24 años $(17,3)$ es el más elevado en todo el período analisado ( Cuadro 2.c).

En resumen, la disminución paulatina de la tasa de mortalidad por causas externas en los adolescentes y jóvenes de Costa Rica se debe a una disminución de las tasas de mortalidad por accidentes de tráfico y homicidios. Costa Rica es uno de los países con tasas mas bajas de mortalidad por homicidios en adolescentes, y el único país donde hay una tendencia a la disminución de las tasas en este grupo etáreo, hasta el año de 1991. Mismo que los valores sean menores en relación a los de otros paises de Latinoamerica, Costa Rica muestra que la mortalidad por homicidios y suicidios entre los hombres de 15 a 24 años, sufriran aumentos considerables entre 1991 y 1995. 
Cuadro 2.a - Tasa* de mortalidad de adolescentes y jóvenes por causas externas, según edad y sexo - Costa Rica 1981-1995

Table 2.a - Mortality rate among adolescents and young adults due to external causes, by age and sex-Costa Rica 1981-1995

\begin{tabular}{ccccccrrrrrr}
\hline Edad & \multicolumn{2}{c}{10 a 14 años } & \multicolumn{2}{c}{15 a 19 años } & \multicolumn{2}{c}{20 a 24 años } & \multicolumn{2}{c}{10 a 19 años } & \multicolumn{2}{c}{15 a 24 años } \\
\hline Años & M & \multicolumn{1}{c}{$\mathrm{F}$} & $\mathrm{M}$ & \multicolumn{1}{c}{$\mathrm{F}$} & \multicolumn{1}{c}{$\mathrm{M}$} & \multicolumn{1}{c}{$\mathrm{F}$} & $\mathrm{M}$ & \multicolumn{1}{c}{$\mathrm{F}$} & $\mathrm{M}$ & $\mathrm{F}$ \\
\hline 1981 & 18,8 & 8,6 & 48,6 & 12,9 & 88,9 & 17,9 & 33,7 & 10,8 & 67,4 & 15,3 \\
1982 & 17,5 & 5,8 & 44,5 & 14,2 & 77,1 & 13,4 & 31,1 & 10,0 & 59,9 & 13,8 \\
1983 & 10,6 & 7,4 & 42,2 & 11,3 & 71,3 & 10,7 & 26,6 & 9,4 & 56,2 & 11,0 \\
1984 & 16,3 & 7,4 & 37,2 & 10,6 & 83,5 & 20,7 & 27,0 & 9,0 & 60,4 & 15,7 \\
1985 & 21,0 & 5,8 & 52,0 & 14,1 & 103,5 & 8,0 & 36,8 & 10,0 & 78,7 & 11,3 \\
1986 & 20,5 & 9,2 & 37,4 & 12,8 & 65,5 & 17,1 & 29 & 11,0 & 51,4 & 14,9 \\
1987 & 19,7 & 14,4 & 47,6 & 6,4 & 87,1 & 14,1 & 33,3 & 10,5 & 67,5 & 10,3 \\
1988 & 12,0 & 4,6 & 46,5 & 13,0 & 73,8 & 13,3 & 28,5 & 8,6 & 60,4 & 13,1 \\
1989 & 20,0 & 7,6 & 44,4 & 13,8 & 63,1 & 14,6 & 31,4 & 10,5 & 53,9 & 14,2 \\
1990 & 16,5 & 8,0 & 49,7 & 10,8 & 77,2 & 9,7 & 31,7 & 9,3 & 63,6 & 10,3 \\
1991 & 12,0 & 6,6 & 40,9 & 11,9 & 59,7 & 14,7 & 25,3 & 9,0 & 50,3 & 13,3 \\
1992 & 17,5 & 7,4 & 53,5 & 13,9 & 80,8 & 8,3 & 34,2 & 10,4 & 66,8 & 11,1 \\
1993 & 16,9 & 6,7 & 48,5 & 8,8 & 72,4 & 15,9 & 26,4 & 7,7 & 59,9 & 12,2 \\
1994 & 17,5 & 5,9 & 44,6 & 12,6 & 85,0 & 13,7 & 30,4 & 9,1 & 63,4 & 13,1 \\
1995 & 17,6 & 7,9 & 55,7 & 13,2 & 99,4 & 18,1 & 35,9 & 10,4 & 75,8 & 15,5 \\
\hline
\end{tabular}

Fuente/Source:OPS. Sistema de Información Técnica

*Tasa/Rate x 100.000 habitantes

M:Masculino/Male

F: Femenino/Female

Cuadro 2.b - Tasa* de mortalidad de adolescentes y jóvenes por accidentes de vehículo a motor, según edad y sexo - Costa Rica 1981-1995

Table 2.b - Mortality rate among adolescents and young adults due to traffic accidents, by age and sex - Costa Rica 1981-1995

\begin{tabular}{ccccccccccc}
\hline Edad & \multicolumn{1}{c}{10 a 14 años } & \multicolumn{2}{c}{15 a 19 años } & \multicolumn{2}{c}{20 a 24 años } & \multicolumn{2}{c}{10 a 19 años } & \multicolumn{2}{c}{15 a 24 años } \\
\hline Años & M & F & \multicolumn{1}{c}{ M } & F & M & F & M & F & M & F \\
\hline 1981 & 5,6 & 2,9 & 16,0 & 1,4 & 34,9 & 5,7 & 10,8 & 2,2 & 24,8 & 3,4 \\
1982 & 5,6 & 2,9 & 15,1 & 3,5 & 30,5 & 2,4 & 10,4 & 3,2 & 22,4 & 3,0 \\
1983 & 2,8 & 1,5 & 7,5 & 2,8 & 18,4 & 3,8 & 5,2 & 2,2 & 12,7 & 3,3 \\
1984 & 2,1 & 2,9 & 13,5 & 4,2 & 21,6 & 7,4 & 8,0 & 3,6 & 17,6 & 5,9 \\
1985 & 4,2 & 2,9 & 18,2 & 4,2 & 36,4 & 3,6 & 11,3 & 3,6 & 27,6 & 4,0 \\
1986 & 5,5 & 5,0 & 10,9 & 2,8 & 26,9 & 6,4 & 8,2 & 3,9 & 18,8 & 4,6 \\
1987 & 7,2 & 6,2 & 15,9 & 1,4 & 32,0 & 4,9 & 11,4 & 3,8 & 24,0 & 3,2 \\
1988 & 4,4 & 2,0 & 14,6 & 5,1 & 18,1 & 4,2 & 9,3 & 3,4 & 16,4 & 4,6 \\
1989 & 7,9 & 2,5 & 11,8 & 5,8 & 23,5 & 6,3 & 9,7 & 4,1 & 17,7 & 6,0 \\
1990 & 4,1 & 3,1 & 13,1 & 2,9 & 31,5 & 6,3 & 8,3 & 3,0 & 22,4 & 4,6 \\
1991 & 4,3 & 2,4 & 14,8 & 4,9 & 18,1 & 3,5 & 8,6 & 3,5 & 16,4 & 4,2 \\
1992 & 5,5 & 2,3 & 15,7 & 3,3 & 28,5 & 2,1 & 10,2 & 2,8 & 21,9 & 2,7 \\
1993 & 3,2 & 3,3 & 14,4 & 3,8 & 25,7 & 4,8 & 8,4 & 3,5 & 19,7 & 4,3 \\
1994 & 6,7 & 2,2 & 19,4 & 3,6 & 28,1 & 5,5 & 12,7 & 2,8 & 23,5 & 4,5 \\
1995 & 7,0 & 3,2 & 18,0 & 4,0 & 36,5 & 6,0 & 12,3 & 3,6 & 26,5 & 5,0 \\
\hline
\end{tabular}

Fuente/Source:OPS. Sistema de Información Técnica

*Tasa/Rate $\times 100.000$ habitantes

M:Masculino/Male

F:Femenino/Female 
Cuadro 2.c - Tasa* de mortalidad de adolescentes y jóvenes por suicidio, según edad y sexo Costa Rica 1981-1995

Table 2.c - Mortality rate among adolescents and young adults due to suicide, by age and sex - Costa Rica 1981-1995

\begin{tabular}{cccccccccccc}
\hline Edad & \multicolumn{1}{c}{10 a 14 años } & \multicolumn{2}{c}{15 a 19 años } & \multicolumn{2}{c}{20 a 24 años } & \multicolumn{2}{c}{10 a 19 años } & \multicolumn{2}{c}{15 a 24 años } \\
\hline Años & M & F & M & F & \multicolumn{1}{c}{ M } & F & M & $F$ & $M$ & F \\
\hline 1981 & 0,0 & 2,2 & 4,9 & 4,3 & 14,3 & 4,1 & 2,4 & 3,2 & 9,3 & 4,2 \\
1982 & 0,0 & 0,0 & 1,4 & 2,1 & 9,2 & 3,9 & 0,7 & 1,1 & 5,1 & 3,0 \\
1983 & 0,7 & 0,0 & 6,1 & 4,2 & 14,7 & 3,1 & 3,5 & 2,2 & 10,2 & 3,7 \\
1984 & 0,7 & 0,7 & 4,1 & 1,4 & 8,6 & 1,5 & 2,4 & 1,1 & 6,4 & 1,5 \\
1985 & 1,4 & 0,7 & 6,8 & 4,9 & 16,1 & 0,7 & 4,1 & 2,9 & 11,5 & 2,9 \\
1986 & 0,7 & 0,7 & 5,4 & 2,1 & 8,3 & 2,9 & 3,1 & 1,4 & 6,8 & 2,5 \\
1987 & 0,0 & 1,4 & 3,4 & 1,4 & 11,6 & 3,5 & 1,7 & 1,4 & 7,5 & 2,5 \\
1988 & 0,0 & 0,0 & 2,4 & 1,4 & 10,7 & 4,2 & 2,0 & 0,7 & 7,5 & 2,8 \\
1989 & 1,2 & 1,3 & 4,9 & 4,3 & 10,7 & 4,2 & 2,9 & 2,7 & 7,8 & 4,3 \\
1990 & 2,4 & 0,6 & 7,6 & 2,9 & 16,1 & 0,7 & 4,8 & 1,7 & 11,9 & 1,8 \\
1991 & 0,6 & 0,0 & 2,7 & 5,6 & 7,4 & 1,4 & 1.5 & 2,6 & 5,0 & 3,5 \\
1992 & 1,1 & 0,0 & 8,2 & 0,0 & 9,9 & 3,4 & 4,4 & 0,0 & 9,0 & 1,7 \\
1993 & 0,0 & 0,0 & 7,8 & 0,6 & 8,6 & 3,4 & 3,7 & 0,3 & 8,2 & 2,0 \\
1994 & 1,5 & 0,0 & 2,9 & 3,0 & 16,3 & 3,4 & 2,2 & 1,4 & 9,1 & 3,2 \\
1995 & 2,0 & 0,5 & 7,1 & 4,0 & 17,3 & 4,0 & 4,5 & 2,2 & 11,8 & 4,0 \\
\hline
\end{tabular}

Fuente/Source:OPS. Sistema de Información Técnica

*Tasa/Rate 100.000 habitantes

M:Masculino/Male

F:Femenino/Female

Cuadro 2.d - Tasa* de mortalidad de adolescentes y jóvenes por homicidio, según edad y sexo Costa Rica 1981-1995

Table 2.d - Mortality rate among adolescents and young adults due to homicide, by age and sex Costa Rica 1981-1995

\begin{tabular}{ccccccccccc}
\hline Edad & \multicolumn{1}{c}{10 a 14 años } & \multicolumn{2}{c}{15 a 19 años } & \multicolumn{2}{c}{20 a 24 años } & \multicolumn{2}{c}{10 a 19 años } & \multicolumn{2}{c}{15 a 24 años } \\
\hline Años & M & F & M & F & \multicolumn{1}{c}{ M } & F & M & $F$ & $M$ & F \\
\hline 1981 & 0,0 & 0,0 & 4,2 & 2,9 & 10,3 & 1,6 & 2,1 & 1,4 & 7,0 & 2,3 \\
1982 & 1,4 & 0,7 & 4,1 & 0,7 & 9,9 & 0,8 & 2,8 & 0,7 & 6,9 & 0,7 \\
1983 & 0,0 & 0,0 & 6,8 & 2,1 & 11,0 & 0,8 & 3,5 & 1,1 & 8,8 & 1,5 \\
1984 & 1,4 & 0,7 & 2,0 & 2,1 & 13,7 & 5,2 & 1,7 & 1,4 & 7,8 & 3,7 \\
1985 & 0,0 & 0,0 & 3,4 & 2,8 & 17,5 & 2,9 & 1,7 & 1,4 & 10,5 & 2,9 \\
1986 & 0,7 & 2,8 & 4,1 & 3,5 & 9,0 & 3,6 & 2,4 & 3,2 & 6,5 & 3,5 \\
1987 & 0,0 & 1,4 & 3,4 & 1,4 & 11,6 & 3,5 & 1,7 & 1,4 & 7,5 & 2,5 \\
1988 & 0,0 & 0,0 & 2,8 & 2,9 & 14,8 & 2,1 & 1,3 & 1,4 & 8,9 & 2,5 \\
1989 & 1,2 & 0,6 & 3,5 & 0,7 & 8,1 & 1,4 & 2,3 & 0,7 & 5,8 & 1,1 \\
1990 & 0,6 & 0,6 & 6,2 & 2,9 & 10,1 & 0,0 & 3,2 & 1,7 & 8,2 & 1,4 \\
1991 & 1,1 & 1,2 & 1,3 & 0,7 & 6,7 & 0,7 & 1,2 & 1,0 & 4,0 & 0,7 \\
1992 & 0,0 & 0,0 & 5,0 & 1,3 & 15,9 & 2,1 & 2,3 & 0,6 & 10,3 & 1,7 \\
1993 & 0,5 & 0,0 & 5,4 & 1,9 & 13,2 & 0,7 & 2,8 & 0,9 & 9,1 & 1,3 \\
1994 & 1,0 & 0,5 & 4,6 & 1,2 & 13,7 & 1,4 & 2,7 & 0,9 & 8,8 & 1,3 \\
1995 & 0,5 & 1,1 & 4,4 & 2,3 & 15,4 & 3,4 & 2,4 & 1,6 & 9,4 & 2,8 \\
\hline
\end{tabular}

Fuente/Source:OPS. Sistema de Información Técnica

*Tasa/Rate x 100.000 habitantes

M:Masculino/Male

F:Femenino/Female 


\section{EL SALVADOR}

Se incluye El Salvador en el estudio a pesar de contar con datos insuficientes por la magnitud de las tasas de mortalidad por causas externas y homicidios que presenta el país en el período 1981- 1991, explicado por el conflicto armado existente entre esas fechas. Sólo se cuenta con información entre los años 1981 y 1984, 1990 y 1991 (Cuadros 1.3 a 1.5 en Anexo y 3.d).

Las tasas de mortalidad por causas externas en la población total disminuyen en un $61 \%$ en hombres y en un $52 \%$ en mujeres entre 1981 y 1991, alcanzando en 1991 cifras de 176,9 por 100.000 en hombres y
31 por 100.000 en mujeres (Cuadro 1.4 y 1.5 en Anexo).

En la población de adolescentes y jóvenes de ambos sexos se observa también una tendencia a la disminución de la mortalidad por causas externas entre 1981 y 1991, de un $38 \%$ en hombres de 10 a 14 años, $65 \%$ en hombres de 15 a 19 y $68 \%$ en hombres de 20 a 24 años (Cuadro 3.a). A pesar de este descenso, las tasas de mortalidad por causas externas en el año 1991 mantienen valores superiores al promedio de los países de las Américas.

La mortalidad por accidentes de tráfico y por suicidio se ha mantenido estacionaria en la población total en el período estudia-

Cuadro 3.a - Tasa* de mortalidad de adolescentes y jóvenes por causas externas, según edad y sexo - El Salvador 1981-1991

Table 3.a - Mortality rate among adolescents and young adults due to external causes, by age and sex - El Salvador 1981-1991

\begin{tabular}{lccccccccccc}
\hline Edad & \multicolumn{2}{c}{ 10 a 14 años } & \multicolumn{2}{c}{15 a 19 años } & \multicolumn{2}{c}{20 a 24 años } & \multicolumn{2}{c}{10 a 19 años } & \multicolumn{2}{c}{15 a 24 años } \\
\hline Años & M & F & M & F & M & F & M & F & M & F \\
\hline 1981 & 51,4 & 30,9 & 496,8 & 88,6 & 1093,1 & 88,7 & 250,8 & 57,2 & 751,6 & 88,6 \\
1982 & 51,7 & 21,4 & 421,7 & 58,4 & 831,6 & 62,4 & 217,7 & 38,2 & 593,9 & 60,1 \\
1983 & 35,4 & 18,2 & 461,7 & 48,8 & 837,8 & 57,2 & 227,5 & 32,1 & 616,7 & 52,5 \\
1984 & 24,7 & 15,7 & 310,0 & 49,6 & 656,5 & 47,5 & 153,5 & 31,1 & 379,0 & 42,7 \\
1990 & 28,8 & 17,3 & 183,5 & 36,3 & 361,0 & 43,0 & 99,3 & 26,2 & 260,7 & 39,2 \\
1991 & 31,7 & 15,9 & 173,4 & 35,3 & 345,3 & 29,7 & 97,1 & 25,0 & 248,8 & 32,9 \\
\hline
\end{tabular}

Fuente/Source:OPS. Sistema de Información Técnica

*Tasa/Rate 100.000 habitantes

M:Masculino/Male

F: Femenino/Female

Cuadro 3.b - Tasa* de mortalidad de adolescentes y jóvenes por accidentes de vehículo a motor, según edad y sexo - El Salvador 1981-1993

Table 3.b - Mortality rate among adolescents and young adults due to traffic accidents, by age and sex-El Salvador 1981-1993

\begin{tabular}{ccccccccccc}
\hline Edad & \multicolumn{2}{c}{ 10 a 14 años } & \multicolumn{2}{c}{ 15 a 19 años } & \multicolumn{2}{c}{20 a 24 años } & \multicolumn{2}{c}{10 a 19 años } & \multicolumn{2}{c}{15 a 24 años } \\
\hline Años & M & F & M & F & M & F & M & F & M & F \\
\hline 1981 & 12,5 & 6,9 & 15,9 & 7,9 & 41,5 & 6,9 & 14,0 & 7,3 & 26,8 & 7,4 \\
1982 & 8,5 & 5,2 & 13,6 & 3,1 & 39,0 & 6,4 & 10,8 & 4,2 & 24,3 & 4,6 \\
1983 & 7,5 & 2,9 & 12,5 & 5,0 & 38,4 & 8,0 & 9,7 & 3,8 & 23,2 & 6,3 \\
1984 & 4,6 & 3,5 & 11,1 & 4,2 & 36,0 & 5,4 & 7,5 & 3,8 & 17,9 & 4,1 \\
1990 & 9,2 & 4,7 & 19,4 & 5,5 & 34,7 & 7,6 & 13,8 & 5,1 & 26,1 & 6,4 \\
1991 & 10,8 & 5,0 & 17,2 & 4,7 & 37,7 & 3,9 & 13,7 & 4,9 & 26,2 & 4,3 \\
1992 & 9,1 & 4,7 & 18,9 & 5,0 & 35,4 & 5,3 & 13,8 & 4,8 & 26,1 & 5,2 \\
1993 & 9,8 & 4,2 & 21,9 & 9,1 & 42,3 & 8,5 & 15,7 & 6,6 & 30,8 & 8,8 \\
\hline
\end{tabular}

Fuente/Source:OPS. Sistema de Información Técnica

*Tasa/Rate $\times 100.000$ habitantes

M:Masculino/Male

F: Femenino/Female 
Cuadro 3.c - Tasa* de mortalidad de adolescentes y jóvenes por suicidio, según edad y sexo - El Salvador $1981-1993$

Table 3.c - Mortality rate among adolescents and young adults due to suicide, by age and sex - El Salvador $1981-1993$

\begin{tabular}{ccccccccccc}
\hline Edad & \multicolumn{1}{c}{ 10 a 14 años } & \multicolumn{2}{c}{ 15 a 19 años } & \multicolumn{2}{c}{20 a 24 años } & \multicolumn{2}{c}{10 a 19 años } & \multicolumn{2}{c}{15 a 24 años } \\
\hline Años & M & F & M & F & M & F & M & F & M & F \\
\hline 1981 & 0,6 & 1,3 & 19,8 & 19,3 & 59,6 & 13,8 & 9,2 & 9,5 & 36,8 & 16,8 \\
1982 & 0,6 & 0,3 & 21,7 & 18,3 & 47,1 & 15,8 & 10,1 & 8,5 & 32,4 & 17,2 \\
1993 & 0,3 & 1,6 & 23,9 & 23,8 & 51,4 & 18,4 & 10,9 & 11,7 & 35,2 & 21,5 \\
1984 & 0,9 & 1,9 & 16,3 & 23,9 & 41,6 & 21,3 & 7,9 & 11,9 & 25,8 & 20,0 \\
1990 & 0,3 & 2,0 & 18,1 & 20,9 & 30,1 & 18,9 & 8,4 & 10,8 & 23,3 & 20,0 \\
1991 & 0,3 & 2,2 & 14,4 & 19,7 & 21,1 & 13,1 & 6,8 & 10,5 & 17,3 & 16,8 \\
1992 & 1,7 & 1,7 & 10,2 & 16,9 & 20,3 & 12,6 & 5,8 & 9,0 & 14,6 & 14,9 \\
1993 & 1,2 & 3,9 & 13,2 & 15,8 & 26,5 & 10,7 & 7,0 & 9,7 & 19,0 & 13,5 \\
\hline
\end{tabular}

Fuente/Source:OPS. Sistema de Información Técnica

*Tasa/Rate $\times 100.000$ habitantes

M:Masculino/Male

F:Femenino/Female

Cuadro 3.d - Tasa* de mortalidad de adolescentes y jóvenes por homicidio, según edad y sexo El Salvador 1991

Table 3.d - Mortality rate among adolescents and young adults due to homicide, by age and sex - EI Salvador 1991

\begin{tabular}{ccccccccccc}
\hline Edad & \multicolumn{2}{c}{10 a 14 años } & \multicolumn{2}{c}{15 a 19 años } & \multicolumn{2}{c}{ 20 a 24 años } & \multicolumn{2}{c}{10 a 19 años } & \multicolumn{2}{c}{15 a 24 años } \\
\hline Años & M & F & M & F & M & F & M & F & M & F \\
\hline 1991 & 4,7 & 2,8 & 109,7 & 3,1 & 244,5 & 4,2 & 53,1 & 2,9 & 168,8 & 3,6 \\
\hline
\end{tabular}

Fuente/Source:OPS. Sistema de Información Técnica

*Tasa/Rate x 100.000 habitantes

M:Masculino/Male

F:Femenino/Female

do. En cambio, en los adolescentes y jóvenes se observa una leve tendencia al descenso de ambos indicadores. Cabe señalar que no hay datos suficientes para realizar un análisis mas detallado (Cuadros 3.b y 3.c).

No hay información respecto a la tendencia de las tasas de homicidio de adolescentes y jóvenes en el período estudiado. Las tasas de mortalidad por homicidio en 1991 en el grupo de 10 a 14 años es de 4,7 por 100.000 en hombres y 2,8 por 100.000 en mujeres; de 15 a 19 años: 109,7 por 100.000 en hombres y 3,1 por 100.000 en mujeres, y de 20 a 24 años: 244,5 por 100.000 en hombres y 4,2 por 100.000 en mujeres (Cuadro 3.d).

En resumen, a pesar de la tendencia al descenso entre 1981 y 1991, El Salvador es un país con una de las tasas mas altas de mortalidad por causas externas y homicidios en la población adolescente y joven, solamente superada por Colombia. Esto se explica principalmente por el conflicto bélico que vivió el país durante el período estudiado.

\section{B. Países de América del Norte}

\section{CANADA}

Las tasas de mortalidad por causas externas son progresivamente descendentes en la población total (Cuadros 1.3 a 1.5 en Anexo). Durante el período estudiado (1980-1995) hay una disminución de un 28\%, alcanzando en 1995 tasas de 45,8 por 100.000. En la población de adolescentes y jóvenes se observa la misma tendencia al descenso en todos los grupos de edad y sexo (Cuadro 4.a), con una disminución de $47 \%$ y $27 \%$ en hombres y mujeres de 10 a 14 años respectivamente; $44 \%$ en el grupo de hombres y $36 \%$ en el grupo de mujeres de 15 a 19 años y $40 \%$ y $46 \%$ de descenso en hom- 
Cuadro 4.a - Tasa* de mortalidad de adolescentes y jóvenes por causas externas, según edad y sexo - Canadá 1980-1995

Table 4.a - Mortality rate among adolescents and young adults due to external causes, by age and sex - Canada 1980-1995

\begin{tabular}{ccccccccccc}
\hline Edad & \multicolumn{1}{c}{10 a 14 años } & \multicolumn{2}{c}{15 a 19 años } & \multicolumn{2}{c}{20 a 24 años } & \multicolumn{2}{c}{10 a 19 años } & \multicolumn{2}{c}{15 a 24 años } \\
\hline Años & M & F & \multicolumn{1}{c}{ M } & F & M & F & M & $F$ & $M$ & F \\
\hline 1980 & 25,3 & 10,8 & 110,2 & 34,7 & 134,5 & 34,2 & 72,0 & 24,0 & 122,3 & 34,4 \\
1981 & 27,0 & 10,8 & 114,2 & 30,5 & 131,3 & 29,6 & 75,1 & 21,7 & 122,9 & 30,0 \\
1982 & 23,8 & 10,9 & 93,9 & 25,0 & 109,5 & 23,0 & 62,2 & 18,6 & 102,0 & 23,9 \\
1983 & 21,2 & 8,5 & 85,0 & 25,2 & 114,8 & 24,2 & 55,7 & 17,5 & 101,0 & 24,7 \\
1984 & 19,3 & 8,7 & 88,1 & 21,4 & 103,2 & 23,8 & 56,0 & 15,4 & 96,4 & 22,7 \\
1985 & 18,9 & 9,3 & 75,5 & 26,3 & 102,7 & 23,0 & 48,6 & 18,2 & 90,6 & 24,5 \\
1986 & 15,5 & 8,6 & 82,2 & 26,1 & 98,5 & 22,8 & 50,1 & 17,6 & 91,2 & 24,3 \\
1987 & 20,6 & 8,1 & 86,1 & 25,6 & 99,5 & 22,3 & 54,3 & 17,1 & 93,5 & 23,8 \\
1988 & 17,8 & 7,5 & 83,5 & 21,9 & 98,9 & 22,5 & 51,4 & 14,9 & 91,8 & 22,3 \\
1989 & 15,0 & 7,7 & 83,7 & 24,3 & 92,9 & 27,2 & 49,9 & 16,1 & 88,6 & 25,8 \\
1990 & 17,6 & 7,7 & 70,4 & 21,2 & 84,9 & 22,2 & 44,3 & 14,6 & 78,0 & 21,8 \\
1991 & 14,8 & 7,1 & 71,4 & 22,1 & 96,0 & 21,3 & 43,2 & 14,7 & 84,2 & 21,7 \\
1992 & 13,6 & 5,7 & 66,3 & 21,5 & 85,4 & 22,7 & 39,9 & 13,6 & 76,1 & 22,1 \\
1993 & 11,9 & 6,9 & 67,5 & 25,0 & 81,3 & 22,2 & 39,5 & 15,9 & 74,6 & 23,5 \\
1994 & 12,6 & 7,6 & 66,6 & 20,8 & 81,5 & 19,4 & 39,2 & 14,1 & 74,2 & 20,1 \\
1995 & 13,4 & 7,9 & 61,5 & 22,3 & 80,3 & 18,5 & 37,3 & 15,0 & 71,0 & 20,4 \\
\hline
\end{tabular}

Fuente/Source:OPS. Sistema de Información Técnica

*Tasa/Rate 100.000 habitantes

M:Masculino/Male

F:Femenino/Female

Cuadro 4.b - Tasa* de mortalidad de adolescentes y jóvenes por accidentes de vehículo a motor, según edad y sexo - Canadá 1980-1995

Table 4.b - Mortality rate among adolescents and young adults due to traffic accidents, by age and sex-Canada 1980-1995

\begin{tabular}{ccccccccccc}
\hline Edad & \multicolumn{3}{c}{10 a 14 años } & \multicolumn{2}{c}{15 a 19 años } & \multicolumn{2}{c}{20 a 24 años } & \multicolumn{2}{c}{10 a 19 años } & \multicolumn{2}{c}{15 a 24 años } \\
\hline Años & M & F & M & F & M & F & M & $F$ & $M$ & F \\
\hline 1980 & 11,2 & 6,5 & 65,0 & 23,7 & 67,4 & 17,9 & 40,8 & 16,0 & 66,2 & 20,8 \\
1981 & 12,7 & 4,7 & 62,5 & 19,0 & 63,9 & 15,8 & 40,2 & 12,6 & 63,2 & 17,4 \\
1982 & 9,7 & 5,9 & 48,0 & 15,7 & 10,5 & 10,5 & 30,7 & 11,3 & 28,3 & 13,0 \\
1983 & 9,5 & 4,4 & 43,3 & 15,9 & 52,5 & 12,1 & 27,8 & 10,6 & 48,2 & 13,9 \\
1984 & 8,4 & 5,0 & 44,9 & 12,5 & 45,1 & 11,2 & 27,9 & 9,0 & 45,0 & 11,8 \\
1985 & 7,4 & 5,4 & 40,5 & 15,9 & 48,1 & 12,6 & 24,8 & 10,9 & 44,7 & 14,1 \\
1986 & 7,4 & 4,1 & 42,8 & 16,1 & 44,1 & 11,2 & 25,8 & 10,3 & 43,5 & 13,4 \\
1987 & 8,7 & 4,4 & 45,3 & 15,5 & 46,8 & 12,1 & 27,5 & 10,1 & 46,1 & 13,6 \\
1988 & 7,4 & 4,6 & 44,0 & 15,4 & 44,4 & 11,5 & 26,1 & 10,1 & 44,2 & 13,3 \\
1989 & 7,2 & 3,8 & 40,0 & 16,5 & 41,9 & 14,6 & 23,9 & 10,3 & 41,0 & 15,4 \\
1990 & 8,0 & 4,5 & 34,0 & 11,9 & 35,2 & 12,5 & 21,1 & 8,2 & 34,6 & 12,2 \\
1991 & 6,5 & 3,5 & 31,9 & 12,4 & 38,7 & 11,0 & 19,3 & 8,0 & 35,4 & 11,7 \\
1992 & 4,8 & 2,3 & 29,2 & 12,1 & 34,1 & 10,1 & 17,0 & 7,2 & 31,7 & 11,0 \\
1993 & 5,7 & 2,8 & 32,8 & 15,4 & 32,9 & 10,7 & 19,1 & 9,1 & 32,8 & 13,0 \\
1994 & 4,5 & 2,9 & 30,7 & 11,7 & 30,0 & 7,8 & 17,4 & 7,2 & 30,3 & 9,7 \\
1995 & 4,3 & 3,6 & 25,0 & 13,8 & 30,0 & 7,7 & 14,6 & 8,6 & 27,5 & 10,7 \\
\hline
\end{tabular}

Fuente/Source:OPS. Sistema de Información Técnica

*Tasa/Rate $\times 100.000$ habitantes

M:Masculino/Male

F: Femenino/Female 
Cuadro 4.c - Tasa* de mortalidad de adolescentes y jóvenes por suicidio, según edad y sexo Canadá 1980-1995

Table 4.c - Mortality rate among adolescents and young adults due to suicide, by age and sex Canada 1980-1995

\begin{tabular}{ccccccccccc}
\hline Edad & \multicolumn{1}{c}{10 a 14 años } & \multicolumn{2}{c}{15 a 19 años } & \multicolumn{2}{c}{20 a 24 años } & \multicolumn{2}{c}{10 a 19 años } & \multicolumn{2}{c}{15 a 24 años } \\
\hline Años & M & F & M & F & M & F & M & F & M & F \\
\hline 1980 & 1,5 & 0,6 & 18,9 & 3,7 & 29,1 & 6,6 & 11,1 & 2,3 & 24,0 & 5,2 \\
1981 & 2,5 & 1,0 & 20,6 & 3,7 & 31,1 & 5,6 & 12,5 & 2,5 & 25.9 & 4,7 \\
1982 & 2,4 & 0,4 & 21,1 & 3,1 & 29,7 & 5,9 & 12,6 & 1,9 & 25,6 & 4,6 \\
1983 & 2,1 & 0,3 & 22,3 & 3,7 & 31,8 & 5,0 & 13,0 & 2,1 & 27,3 & 4,4 \\
1984 & 2,4 & 0,4 & 20,5 & 3,1 & 29,9 & 5,0 & 12,0 & 1,9 & 25,7 & 4,1 \\
1985 & 1,3 & 0,6 & 17,9 & 3,5 & 29,0 & 4,1 & 10,0 & 2,1 & 24,0 & 3,8 \\
1986 & 2,0 & 0,6 & 19,5 & 4,4 & 29,4 & 5,5 & 11,1 & 2,5 & 25,0 & 5,0 \\
1987 & 2,6 & 0,6 & 20,0 & 4,5 & 27,1 & 4,9 & 11,6 & 2,6 & 23,9 & 4,7 \\
1988 & 2,4 & 0,4 & 21,3 & 3,2 & 28,7 & 5,8 & 12,1 & 1,8 & 25,3 & 4,6 \\
1989 & 2,0 & 0,7 & 22,0 & 3,1 & 27,5 & 5,7 & 12,1 & 1,9 & 24,9 & 4,5 \\
1990 & 2,4 & 0,7 & 18,4 & 4,5 & 27,7 & 4,8 & 10,5 & 2,6 & 23,3 & 4,7 \\
1991 & 1,9 & 1,0 & 22,0 & 3,8 & 30,4 & 3,9 & 12,0 & 2,4 & 26,3 & 3,8 \\
1992 & 2,6 & 0,8 & 20,1 & 5,4 & 29,4 & 6,7 & 11,4 & 3,1 & 24,9 & 6,1 \\
1993 & 2,6 & 1,9 & 19,6 & 4,7 & 28,7 & 4,9 & 11,0 & 3,3 & 24,3 & 4,8 \\
1994 & 3,7 & 1,2 & 20,8 & 5,0 & 29,5 & 5,8 & 12,1 & 3,1 & 29,9 & 5,4 \\
1995 & 2,1 & 2,1 & 21,4 & 4,9 & 27,9 & 4,9 & 11,7 & 3,5 & 24,7 & 4,9 \\
\hline
\end{tabular}

Fuente/Source:OPS. Sistema de Información Técnica

*Tasa/Rate x 100.000 habitantes

M:Masculino/Male

F:Femenino/Female

Cuadro 4.d - Tasa* de mortalidad de adolescentes y jóvenes por homicidio, según edad y sexo Canadá 1980-1995

Table 4.d - Mortality rate among adolescents and young adults due to homicide, by age and sex Canada 1980-1995

\begin{tabular}{ccccccccccc}
\hline Edad & \multicolumn{2}{c}{10 a 14 años } & \multicolumn{2}{c}{15 a 19 años } & \multicolumn{2}{c}{20 a 24 años } & \multicolumn{2}{c}{10 a 19 años } & \multicolumn{2}{c}{15 a 24 años } \\
\hline Años & M & F & M & F & M & F & M & F & M & F \\
\hline 1980 & 0,2 & 0,3 & 1,4 & 0,9 & 3,1 & 2,2 & 0,8 & 0,7 & 2,2 & 1,6 \\
1981 & 1,1 & 1,0 & 2,3 & 1,6 & 4,0 & 2,9 & 1,8 & 1,3 & 3,2 & 2,3 \\
1982 & 0,7 & 0,4 & 3,2 & 2,1 & 4,2 & 1,6 & 2,1 & 1,3 & 3,8 & 1,8 \\
1983 & 0,3 & 0,6 & 0,2 & 1,9 & 4,3 & 1,8 & 1,2 & 1,3 & 3,2 & 1,8 \\
1984 & 0,5 & 0,4 & 2,5 & 0,9 & 4,0 & 2,5 & 1,6 & 0,7 & 3,3 & 1,8 \\
1985 & 1,2 & 0,6 & 2,6 & 1,5 & 3,4 & 2,2 & 1,9 & 1,1 & 3,0 & 1,9 \\
1986 & 0,7 & 0,8 & 2,2 & 1,3 & 3,3 & 2,0 & 1,5 & 1,1 & 2,8 & 1,7 \\
1987 & 0,6 & 1,0 & 1,9 & 1,7 & 4,5 & 2,0 & 1,3 & 1,3 & 3,3 & 1,9 \\
1988 & 0,6 & 0,1 & 1,5 & 1,1 & 3,4 & 1,9 & 1,1 & 0,6 & 2,5 & 1,5 \\
1989 & 0,3 & 0,4 & 3,9 & 1,4 & 3,8 & 2,7 & 2,2 & 0,9 & 3,9 & 2,1 \\
1990 & 0,4 & 0,3 & 2,9 & 1,8 & 4,0 & 1,5 & 1,7 & 1,1 & 3,5 & 1,6 \\
1991 & 0,3 & 1,3 & 2,2 & 2,2 & 5,4 & 2,3 & 1,3 & 1,8 & 3,9 & 2,3 \\
1992 & 0,5 & 0,3 & 2,8 & 1,0 & 4,1 & 2,1 & 1,7 & 0,6 & 3,5 & 1,5 \\
1993 & 0,0 & 0,3 & 2,4 & 1,2 & 3,7 & 2,1 & 1,2 & 0,7 & 3,1 & 1,6 \\
1994 & 0,3 & 0,7 & 2,9 & 1,3 & 4,0 & 2,3 & 1,6 & 1,0 & 3,5 & 1,8 \\
1995 & 0,6 & 0,3 & 1,9 & 0,8 & 4,2 & 1,7 & 1,2 & 0,6 & 3,1 & 1,3 \\
\hline
\end{tabular}

Fuente/Source:OPS. Sistema de Información Técnica

*Tasa/Rate x 100.000 habitantes

M:Masculino/Male

F:Femenino/Female 
bres y mujeres de 20 a 24 años respectivamente. La mayor tasa de mortalidad por causas externas en 1995 la presenta el grupo de varones de 20 a 24 años, con cifras de 80,3 por 100.000 .

La principal causa determinante de la mortalidad por causas externas en Canadá son los accidentes de tráfico. En la población total hay un descenso de $52 \%$ en la mortalidad por esta causa entre los años extremos del período estudiado. La tasa de mortalidad por accidentes de tráfico es también decreciente en todas las categorías de edad y sexo de los adolescentes y jóvenes (Cuadro 4b). Los porcentajes de descenso en hombres alcanzan un $62 \%$ en el grupo de 10 a $14 ; 61 \%$ en el grupo de 15 a 19 y $55 \%$ en el grupo de 20 a 24 años. Cifras semejantes de disminución se observan en los respectivos grupos de mujeres.

La mortalidad por suicidio es el segundo componente mas importante de la mortalidad por causas externas en Canadá. Las tasas de mortalidad por suicidio en la población total és practicamente estable entre 1980 y 1995, alcanzando valores de 13,4 por 100.000 en 1995 (Cuadro 1.9 en Anexo).

En el grupo de adolescentes y jóvenes de Canadá las tasas de mortalidad por suicidios en el grupo de 10 a 14 años, en ambos sexos, en el período estudiado, alcanzan tasas de 2,1 por 100.000 en 1995 (Cuadro 4.c). En la población de 15 a 19 años las tasas han aumentado en $13 \%$ entre los hombres y $32 \%$ entre las mujeres entre las mismas fechas, alcanzando tasas de 21,4 por 100.000 en hombres y 4,9 por 100.000 en mujeres en 1995. Los jóvenes de 20 a 24 años presentan las tasas de mortalidad por suicidio mas altas de este grupo. Las tasas se han mantenido estables alrededor de 27,9 por 100.000 en los hombres de 20 a 24 años y 4,9 por 100.000 en las mujeres del mismo grupo etáreo.

La tasa de mortalidad por homicidio es baja en Canadá a todos los grupos estudiados. En la población total la tasa disminuyó en un $15 \%$ (de 2 por 100.000 a 1,7 por 100.000), entre 1980 y 1995 (Cuadro 1.12 en Anexo). En el grupo de adolescentes y jóve- nes las tasas se han mantenido estacionarias entre las mismas fechas, alcanzando en 1995 tasas de 0,6 y 0,3 por 100.000 en hombres y mujeres de 10 a 14 años; 1,9 y 0,8 por 100.000 en hombres y mujeres de 15 a 19 años; 4,2 y 1,7 por 100.000 en hombres y mujeres de 20 a 24 años (Cuadro 4.d).

En resumen, Canadá es uno de los países con menores tasas de mortalidad por causas externas y homicidios en adolescentes de la región. La disminución de la mortalidad por causas externas en los adolescentes y jóvenes de Canadá entre 1980 y 1995 se debe principalmente a la disminución de la mortalidad por accidentes de tráfico. Sin embargo, Canadá es el país con mayores tasas de suicidio entre adolescentes y jóvenes entre los países analizados en este estudio.

\section{EEUU DE NORTEAMERICA}

Al igual que en Canadá, las tasas de mortalidad por causas externas son progresivamente descendentes en la población total, aunque en menor porcentaje (Cuadros 1.3 a 1.5 en Anexo). En el período estudiado, 1980 a 1997, hay una disminución de un $22 \%$, llegando a una tasa de 55,1 por 100.000 en 1997.

En todos los grupos de edad y sexo de adolescentes y jóvenes de EEUU se observa una tendencia leve y progresiva a la disminución de las tasas de mortalidad por causas externas (Cuadro 5.a). En el grupo de 10 a 14 años hay un descenso de $33 \%$ en hombres y $20 \%$ en mujeres; en el grupo de 15 a 19 años hay un descenso de $24 \%$ en hombres y $16 \%$ en mujeres y, en el grupo de 20 a 24 años, un descenso de $30 \%$ en ambos sexos.

El principal componente de la tasa de mortalidad por causas externas en EEUU es la mortalidad por accidentes de tráfico. Entre los años 1980 y 1997 ha habido un 32\% de disminución de esta tasa en la población total. También hay una progresiva disminución de las tasas de mortalidad por accidentes de tráfico en todos los subgrupos de edad y sexo en adolescentes y jóvenes. En 
Cuadro 5.a - Tasa* de mortalidad de adolescentes y jóvenes por causas externas, según edad y sexo - Estados Unidos 1980-1997

Table 5.a - Mortality rate among adolescents and young adults due to external causes, by age and sex-United States 1980-1997

\begin{tabular}{ccrrrrrrrrr}
\hline Edad & \multicolumn{2}{c}{10 a 14 años } & \multicolumn{2}{c}{15 a 19 años } & \multicolumn{2}{c}{20 a 24 años } & \multicolumn{2}{c}{10 a 19 años } & \multicolumn{2}{c}{15 a 24 años } \\
\hline Años & M & \multicolumn{1}{c}{$\mathrm{F}$} & \multicolumn{1}{c}{ M } & $\mathrm{F}$ & $\mathrm{M}$ & $\mathrm{F}$ & $\mathrm{M}$ & $\mathrm{F}$ & $\mathrm{M}$ & $\mathrm{F}$ \\
\hline 1980 & 24,4 & 10,8 & 118,7 & 36,3 & 166,0 & 39,0 & 75,0 & 24,5 & 142,5 & 37,6 \\
1981 & 24,2 & 10,4 & 106.3 & 33,3 & 154,7 & 37,4 & 68,2 & 22,8 & 130,9 & 35,4 \\
1982 & 22,5 & 10,1 & 99.8 & 29.9 & 143,0 & 34,6 & 63,7 & 20,7 & 122,1 & 32,4 \\
1983 & 22,3 & 9,7 & 92,6 & 29,5 & 132,6 & 34,1 & 59,5 & 20,2 & 113.5 & 32,6 \\
1984 & 22,3 & 10,4 & 92,8 & 31,1 & 134,7 & 35,1 & 59,3 & 21,3 & 113,1 & 33,3 \\
1985 & 22,9 & 9,9 & 94,2 & 31,8 & 133,3 & 33,0 & 60,0 & 21,3 & 114,9 & 32,4 \\
1986 & 22,8 & 9,6 & 105,3 & 34,9 & 139,5 & 34,3 & 65,4 & 22,7 & 123,4 & 34,6 \\
1987 & 22,6 & 8,7 & 101,4 & 34,9 & 131,8 & 33,5 & 63,2 & 22,2 & 117,5 & 34,1 \\
1988 & 21,1 & 10,2 & 106,4 & 35,3 & 134,1 & 32,8 & 64,9 & 23,1 & 121,0 & 34,0 \\
1989 & 20,8 & 9,7 & 103,1 & 34,8 & 127,9 & 30,8 & 62,9 & 22,6 & 116,0 & 32,7 \\
1990 & 19,7 & 9,4 & 108,3 & 32,6 & 135.4 & 30,3 & 64,7 & 21,2 & 122,3 & 31,4 \\
1991 & 20,9 & 8,7 & 105,6 & 31,9 & 136,9 & 32,3 & 63,7 & 20,4 & 121,7 & 32,1 \\
1992 & 19,6 & 8,9 & 99,5 & 29,5 & 131,2 & 29,7 & 59,5 & 19,2 & 115,7 & 29,6 \\
1993 & 20,0 & 9,7 & 104,7 & 30,7 & 136,2 & 30,6 & 61.9 & 20,1 & 120,8 & 30,7 \\
1994 & 19,8 & 9,6 & 107,4 & 30,6 & 133,1 & 29,9 & 62,8 & 19,9 & 120,5 & 30,2 \\
1995 & 18,9 & 9,2 & 100,9 & 32,2 & 129,8 & 28,5 & 58,4 & 20,3 & 115,4 & 30,3 \\
1996 & 17,0 & 8,7 & 96,6 & 31,3 & 122,1 & 27,0 & 55,3 & 19,6 & 109,2 & 29,2 \\
1997 & 16,3 & 8,6 & 89,7 & 30,6 & 116,3 & 27,2 & 51,8 & 19,2 & 102,8 & 28,9 \\
\hline
\end{tabular}

Fuente/Source:OPS. Sistema de Información Técnica

*Tasa/Rate x 100.000 habitantes

M:Masculino/Male

F:Femenino/Female

Cuadro 5.b - Tasa* de mortalidad de adolescentes y jóvenes por accidentes de vehículo a motor, según edad y sexo - Estados Unidos 1980-1997

Table 5.b - Mortality rate among adolescents and young adults due to traffic accidents, by age and sex- United States 1980-1997

\begin{tabular}{ccccccccccc}
\hline Edad & \multicolumn{2}{c}{10 a 14 años } & \multicolumn{2}{c}{15 a 19 años } & \multicolumn{2}{c}{20 a 24 años } & \multicolumn{2}{c}{10 a 19 años } & \multicolumn{2}{c}{15 a 24 años } \\
\hline Años & M & F & M & F & M & F & M & F & M & F \\
\hline 1980 & 10,0 & 5,4 & 61,3 & 22,6 & 71,7 & 18,7 & 37,5 & 14,6 & 66,5 & 20,6 \\
1981 & 9,6 & 5,1 & 54,0 & 20,9 & 66,9 & 18,3 & 33,4 & 13,6 & 60,5 & 19,6 \\
1982 & 8,9 & 5,0 & 49,0 & 18,2 & 59,6 & 16,1 & 30,3 & 12,1 & 54,5 & 17.1 \\
1983 & 8,7 & 4,7 & 45,8 & 18,2 & 55,5 & 16,0 & 28,3 & 11,9 & 50,9 & 17,0 \\
1984 & 8,7 & 5,3 & 47,7 & 19,3 & 58,4 & 17,0 & 29,1 & 12,6 & 53,4 & 18,1 \\
1985 & 8,8 & 5,1 & 46,2 & 20,1 & 57,3 & 16,7 & 28,2 & 12,9 & 52,1 & 18,3 \\
1986 & 9,1 & 5,0 & 53,1 & 22,2 & 60,6 & 17,1 & 31,8 & 13,9 & 57,1 & 19,5 \\
1987 & 9,5 & 4,5 & 51,3 & 22,6 & 56,5 & 17.0 & 31,0 & 13,8 & 54,0 & 19,6 \\
1988 & 8,7 & 5,2 & 51,1 & 22,9 & 57,5 & 17,1 & 30,5 & 14,3 & 54,5 & 19,9 \\
1089 & 8,0 & 4,8 & 45,7 & 22,6 & 51,1 & 15,8 & 27,3 & 13,9 & 48,5 & 19,1 \\
1990 & 7,1 & 4,9 & 45,1 & 20,0 & 52,3 & 15,8 & 26,4 & 12,6 & 48,8 & 17,8 \\
1991 & 7,7 & 4,2 & 39,7 & 19,7 & 49,2 & 15,9 & 23,8 & 12,0 & 44,6 & 17,7 \\
1992 & 6,8 & 3,9 & 35,3 & 18,1 & 43,2 & 14,2 & 21,1 & 11,0 & 39,3 & 16,1 \\
1993 & 7,1 & 4,5 & 37,5 & 17,6 & 44,3 & 14.3 & 22,1 & 11,0 & 41,0 & 15,9 \\
1994 & 7,2 & 4,8 & 38,6 & 19,1 & 43,6 & 15,0 & 22,6 & 11,8 & 41,2 & 17,0 \\
1995 & 6,8 & 4,7 & 36,9 & 20,1 & 45,2 & 14,1 & 21,3 & 12,1 & 41,1 & 17,1 \\
1996 & 6,5 & 4,3 & 38,2 & 19,6 & 43,4 & 14,3 & 21,8 & 11,7 & 40,7 & 17,0 \\
1997 & 6,1 & 4,6 & 35,8 & 19,8 & 40,4 & 14,2 & 20,5 & 12,0 & 38,0 & 17,0 \\
\hline
\end{tabular}

Fuente/Source:OPS. Sistema de Información Técnica

*Tasa/Rate $\times 100.000$ habitantes

M:Masculino/Male

F:Femenino/Female 
Cuadro 5.c - Tasa* de mortalidad de adolescentes y jóvenes por suicidio, según edad y sexo Estados Unidos 1980-1997

Table 5.c - Mortality rate among adolescents and young adults due to suicide, by age and sexUnited States 1980-1997

\begin{tabular}{lcccccccccc}
\hline Edad & \multicolumn{1}{c}{ 10 a 14 años } & \multicolumn{2}{c}{15 a 19 años } & \multicolumn{2}{c}{20 a 24 años } & \multicolumn{2}{c}{10 a 19 años } & \multicolumn{2}{c}{15 a 24 años } \\
\hline Años & M & F & M & F & M & F & M & F & M & F \\
\hline 1980 & 1,2 & 0,3 & 13,8 & 3,0 & 26,2 & 5,5 & 7,9 & 1,8 & 20,0 & 4,3 \\
1981 & 1,3 & 0,5 & 13,4 & 3,5 & 25,5 & 5,6 & 7,8 & 2,1 & 19,5 & 4,6 \\
1982 & 1,8 & 0,5 & 13,8 & 3,1 & 25,0 & 5,2 & 8,2 & 1,9 & 19,6 & 4,2 \\
1983 & 1,6 & 1,6 & 13,7 & 3,2 & 24,0 & 5,2 & 8,0 & 2,0 & 19,1 & 4,3 \\
1984 & 1,9 & 0,6 & 14,1 & 3,5 & 25,8 & 5,2 & 8,3 & 2,2 & 20,3 & 4,4 \\
1985 & 2,3 & 0,9 & 16,0 & 3,7 & 25,9 & 4,9 & 9,4 & 2,4 & 21,2 & 4,4 \\
1986 & 2,3 & 0,7 & 16,7 & 3,9 & 25,9 & 4,9 & 9,7 & 2,3 & 21,6 & 4,4 \\
1987 & 2,3 & 0,6 & 16,6 & 4,3 & 25,0 & 4,4 & 9,6 & 2,5 & 21,0 & 4,3 \\
1988 & 2,0 & 0,7 & 18,2 & 4,5 & 24,4 & 4,0 & 10,3 & 2,7 & 21,5 & 4,2 \\
1989 & 2,1 & 0,7 & 17,9 & 4,3 & 24,9 & 4,1 & 10,2 & 2,5 & 21,5 & 4,2 \\
1990 & 2,2 & 0,8 & 18,1 & 3,7 & 25,6 & 4,1 & 10,3 & 2,3 & 22,0 & 3,9 \\
1991 & 2,3 & 0,7 & 17,5 & 3,6 & 25,8 & 4,0 & 10,0 & 2,2 & 21,8 & 3,8 \\
1992 & 2,5 & 0,9 & 17,3 & 3,3 & 26,1 & 4,0 & 9,9 & 2,1 & 21,8 & 3,7 \\
1993 & 2,5 & 1,0 & 17,4 & 3,8 & 27,1 & 4,5 & 9,9 & 2,4 & 22,4 & 4,1 \\
1994 & 2,5 & 1,0 & 18,4 & 3,5 & 28,4 & 4,0 & 10,3 & 2,2 & 23,5 & 3,7 \\
1995 & 2,6 & 0,8 & 17,8 & 3,2 & 27,7 & 4,3 & 9,9 & 2,0 & 22,7 & 3,7 \\
1996 & 2,2 & 0,8 & 16,2 & 3,6 & 24,6 & 3,6 & 9,0 & 2,2 & 20,4 & 3,6 \\
1997 & 2,3 & 0,8 & 15,9 & 3,5 & 22,9 & 3,6 & 8,9 & 2,1 & 19,3 & 3,5 \\
\hline
\end{tabular}

Fuente/Source: OPS. Sistema de Información Técnica

*Tasa/Rate x 100.000 habitantes

M:Masculino/Male

F:Femenino/Female

Cuadro 5.d - Ttasa* de mortalidad de adolescentes y jóvenes por homicidio, según edad y sexo Estados Unidos 1980-1997

Table 5.d - Mortality rate among adolescents and young adults due to homicide, by age and sexUnited States 1980-1997

\begin{tabular}{ccccccccccc}
\hline Edad & \multicolumn{2}{c}{10 a 14 años } & \multicolumn{2}{c}{15 a 19 años } & \multicolumn{2}{c}{20 a 24 años } & \multicolumn{2}{c}{10 a 19 años } & \multicolumn{2}{c}{15 a 24 años } \\
\hline Años & M & F & M & F & M & F & M & F & M & F \\
\hline 1980 & 1,6 & 1,3 & 16,2 & 4,9 & 32,3 & 8,2 & 9,4 & 3,3 & 24,3 & 6,6 \\
1981 & 1,7 & 1,4 & 15,3 & 4,5 & 29,9 & 7,7 & 9,0 & 3,1 & 22,7 & 6,1 \\
1982 & 1,4 & 1,3 & 14,5 & 4,5 & 27,2 & 7,2 & 8,4 & 3,0 & 21,0 & 5,9 \\
1983 & 1,6 & 0,9 & 12,6 & 4,0 & 24,6 & 6,6 & 7,4 & 2,5 & 18,9 & 5,4 \\
1984 & 1,7 & 1,5 & 12,1 & 4,2 & 23,1 & 7,2 & 7,2 & 2,9 & 17,9 & 5,8 \\
1985 & 1,9 & 1,1 & 13,2 & 3,9 & 23,6 & 6,2 & 7,7 & 2,6 & 18,7 & 5,1 \\
1986 & 1,7 & 1,2 & 15,4 & 4,8 & 27,7 & 7,3 & 8,8 & 3,1 & 21,9 & 6,2 \\
1987 & 1,9 & 1,2 & 15,7 & 4,5 & 26,9 & 7,3 & 9,0 & 2,9 & 21,6 & 6,0 \\
1988 & 2,0 & 1,3 & 19,0 & 4,5 & 28,9 & 7,2 & 10,7 & 2,9 & 24,2 & 5,9 \\
1989 & 2,4 & 1,4 & 22,3 & 4,6 & 30,9 & 7,0 & 12,6 & 3,1 & 26,8 & 5,9 \\
1990 & 2,6 & 1,5 & 28,2 & 5,4 & 37,4 & 7,0 & 15,6 & 3,5 & 32,9 & 6,3 \\
1991 & 3,0 & 1,4 & 31,9 & 5,4 & 41,8 & 8,3 & 17,6 & 3,4 & 37,0 & 6,9 \\
1992 & 3,3 & 1,7 & 31,7 & 5,1 & 42,3 & 7,6 & 17,5 & 3,4 & 37,2 & 6,4 \\
1993 & 3,3 & 1,8 & 34,2 & 5,8 & 43,8 & 8,0 & 18,6 & 3,8 & 39,1 & 6,9 \\
1994 & 3,0 & 1,5 & 34,7 & 5,3 & 41,7 & 7,1 & 18,6 & 3,4 & 38,3 & 6,2 \\
1995 & 2,9 & 1,3 & 30,6 & 5,9 & 37,9 & 6,2 & 16,3 & 3,5 & 34,2 & 6,1 \\
1996 & 2,2 & 1,2 & 27,2 & 4,7 & 34,7 & 5,5 & 14,3 & 2,9 & 30,9 & 5,1 \\
1997 & 2,0 & 0,9 & 23,8 & 4,3 & 33,9 & 5,3 & 12,5 & 2,5 & 28,8 & 4,8 \\
\hline
\end{tabular}

Fuente/Source:OPS. Sistema de Información Técnica

*Tasa/Rate x 100.000 habitantes

M:Masculino/Male

F:Femenino/Female 
el grupo de hombres de 15 a 24 años hay un $43 \%$ de descenso y $18 \%$ de descenso en las mujeres de igual edad, en el mismo período (Cuadro 5.b).

El segundo determinante de la mortalidad por causas externas en EEUU en la población total es el suicidio, el que se ha mantenido estable alcanzando tasas de 11,2 por 100.000 en 1997 en esta población (Cuadro 1.9 en Anexo). En cambio, el homicidio en la población total de EEUU ha sufrido un leve ascenso de un $14 \%$, desde el año 1985 hasta 1994. Pero, de 1995 a 1997 los homicidios llegan a sus menores valores, en el período estudiado, de 1980 a1997. En ese último año fue de 7,3 que representa un descenso de $34 \%$ en relación a la tasa de 1980 (Cuadro 1.12 en Anexo).

En la población de adolescentes y jóvenes de EEUU, el segundo factor determinante de la tasa de mortalidad por causas externas es la mortalidad por homicidios, que ha sufrido un progresivo aumento en todos los subgrupos de edad y sexo entre los años 1980 y 1994. En la población de 10 a 14 años hay un aumento de $88 \%$ y $15 \%$ en hombres y mujeres respectivamente; en el grupo de 15 a 19 años, un aumento de $114 \%$ y $8 \%$; y en el grupo de hombres de 20 a 24 años un aumento de $29 \%$, entre esas fechas. El incremento de la tasa de mortalidad por homicidios en adolescentes y jóvenes de EEUU ha sido dramático entre las fechas 1985 y 1994, en que las tasas aumentan en un $163 \%$ (de 13,2 a 34,7 por 100.000) en el grupo de hombres de 15 a 19 años y en un $77 \%$ (de 23,6 a 41,7 por 100.000) en el grupo de hombres de 20 a 24 años (Cuadro 5.d).

Entretanto, siguiendo la tendencia de disminución de las tasas de mortalidad por homicidios en la población total, la misma también disminuye entre la población adolescente y joven, entre 1994 y 1997. Entre los hombres de 15 a19 años, las tasas de homicidio disminuyen de 34,7 en 1994, para 23,8 em 1997, o sea, $31 \%$ en cuatro años. Lo mismo acontece entre los hombres de 20 a 24 años, de 41,7 em 1994, para 33,9 en 1997, significando una disminuición de $19 \%$. También entre las mujeres de los respecti- vos grupos etários, la tasa de mortalidad por homicidios disminuye, aun cuando sus valores sean considerablemente menores que el de los hombres (Cuadro 5.d).

La tasa de mortalidad por suicidio también ha aumentado en EEUU entre los años 1980 y 1994. El grupo de hombres y mujeres de 10 a 14 años doblan su tasa en el período estudiado (de 1,2 a 2,5 por 100.000 en los hombres y de 0,3 a 1,0 por 100.000 en las mujeres). En el grupo de hombres de 15 a 19 años la tasa aumentó en un 33\% y entre los hombres de 20 a 24 años sólo aumentó en un 9\%. Las tasas en las mujeres de 15 a 24 años sufren un leve descenso(14\%) entre los mismos años (Cuadro 5.c).

A partir de 1995 la tasa de mortalidad por suicidios disminuye, mantiendose practicamente estable entre las mujeres de los grupos etários analisados; de 10 y 14 años (0,8 por 100.000); de 15 a19 años (3,5 por 1000.000); y, de 20 a 24 años (3,6 por 100.000) en 1997. El descenso entre los hombres de 15 a 19 años fue de $14 \%$ y en los de 20 a 24 años 19\%, entre los respectivos años (Cuadro 5.c).

En resumen, la disminución de la mortalidad por causas externas en el grupo de adolescentes y jóvenes de EEUU se explica por la disminución de los accidentes de tráfico. La mortalidad por homicidios en este grupo ha aumentado francamente de 1985 y 1994, especialmente en el grupo de hombres de 15 a 19 años. A partir de 1995 las tasas de mortalidad por homicidios sufren redución, volviendo las tasas de mortalidad por accidentes de trafico a presentar mayor peso en la mortalidad por causas externas entre adolescentes y jovenes americanos.

\section{MEXICO}

La mortalidad por causas externas ha disminuido progresivamente en el período estudiado en México (1980-1995) en un 39\% en la población total y en un $44 \%$ entre los jóvenes varones de 15 a 24 años. Las reducciones son mayores a mayor edad y entre las mujeres (Cuadros 1.3 a 1.5 en Anexo y 6.a). 
Cuadro 6.a - Tasa* de mortalidad de adolescentes y jóvenes por causas externas, según edad y sexo - México 1980-1995

Table 6.a - Mortality rate among adolescents and young adults due to external causes, by age and sex-Mexico 1980-1995

\begin{tabular}{ccccccccccc}
\hline Edad & \multicolumn{1}{c}{ 10 a 14 años } & \multicolumn{2}{c}{ 15 a 19 años } & \multicolumn{2}{c}{20 a 24 años } & \multicolumn{2}{c}{10 a 19 años } & \multicolumn{2}{c}{15 a 24 años } \\
\hline Años & M & F & M & F & M & F & M & F & M & F \\
\hline 1980 & 48,1 & 16,9 & 141,3 & 28,7 & 257,2 & 33,9 & 90,3 & 22,3 & 192,9 & 31,1 \\
1981 & 48,0 & 18,1 & 141,0 & 28,3 & 260,7 & 36,9 & 90,1 & 22,7 & 194,4 & 32,1 \\
1982 & 45,3 & 16,0 & 131,3 & 26,2 & 248,2 & 33,6 & 84,2 & 20,6 & 183,6 & 29,5 \\
1983 & 35,9 & 12,7 & 104,9 & 20,9 & 205,7 & 28,2 & 67,1 & 16,5 & 150,0 & 24,2 \\
1984 & 35,9 & 12,8 & 102,2 & 18,2 & 194,8 & 26,8 & 66,1 & 15,3 & 138,3 & 21,4 \\
1985 & 37,0 & 13,2 & 105,0 & 24,9 & 193,6 & 31,8 & 68,3 & 18,6 & 139,7 & 27,2 \\
1986 & 36,3 & 12,1 & 105,5 & 19,6 & 185,8 & 24,1 & 68,7 & 15,6 & 145,2 & 20,4 \\
1987 & 35,6 & 12,1 & 100,6 & 16,7 & 182,0 & 21,9 & 66,6 & 14,3 & 137,1 & 19,1 \\
1988 & 33,6 & 11,6 & 96,2 & 17,5 & 165,0 & 21,1 & 64,2 & 14,5 & 119,5 & 18,0 \\
1989 & 32,9 & 12,4 & 94,1 & 17,6 & 155,4 & 19,8 & 63,4 & 15,0 & 114,5 & 17,5 \\
1990 & 32,0 & 11,9 & 91,6 & 17,9 & 144,3 & 20,3 & 61,9 & 14,9 & 108,9 & 17,9 \\
1991 & 31,0 & 11,6 & 94,3 & 18,8 & 150,9 & 19,4 & 62,8 & 15,2 & 119,5 & 18,9 \\
1992 & 28,4 & 10,7 & 96,6 & 17,5 & 152,2 & 18,3 & 62,4 & 14,1 & 137,1 & 17,9 \\
1993 & 26,4 & 9,8 & 91,3 & 17,3 & 139,0 & 16,8 & 58,4 & 13,5 & 114,8 & 17,1 \\
1994 & 24,7 & 10,0 & 87,8 & 16,7 & 149,7 & 16,7 & 56,0 & 13,4 & 117,2 & 16,7 \\
1995 & 23,1 & 10,0 & 82,0 & 17,1 & 138,1 & 17,3 & 52,3 & 13,5 & 109,0 & 17,2 \\
\hline
\end{tabular}

Fuente/Source:OPS. Sistema de Información Técnica

*Tasa/Rate x 100.000 habitantes

M:Masculino/Male

F: Femenino/Female

Cuadro 6.b - Tasa* de mortalidad de adolescentes y jóvenes por accidentes de vehículo a motor, según edad y sexo - México 1980-1995

Table 6.b - Mortality rate among adolescents and young adults due to traffic accidents, by age and sex-Mexico 1980-1995

\begin{tabular}{ccccccccccr}
\hline Edad & \multicolumn{2}{c}{10 a 14 años } & \multicolumn{2}{c}{15 a 19 años } & \multicolumn{2}{c}{20 a 24 años } & \multicolumn{2}{c}{10 a 19 años } & \multicolumn{2}{c}{15 a 24 años } \\
\hline Años & M & F & M & F & M & F & M & $F$ & $M$ & F \\
\hline 1980 & 13,6 & 5,1 & 31,9 & 8,2 & 58,8 & 9,9 & 21,9 & 6,5 & 43,9 & 8,9 \\
1981 & 13,4 & 5,9 & 33,3 & 8,6 & 62,2 & 12,1 & 22,4 & 7,1 & 46,2 & 10,2 \\
1982 & 13,7 & 4,6 & 28,7 & 8,7 & 55,9 & 9,7 & 20,5 & 6,5 & 40,9 & 9,1 \\
1983 & 9,9 & 4,1 & 23,5 & 5,8 & 44,5 & 8,5 & 16,0 & 4,9 & 32,9 & 7,0 \\
1984 & 9,5 & 3,7 & 20,1 & 4,9 & 38,7 & 7,0 & 14,3 & 4,2 & 27,4 & 5,7 \\
1985 & 8,8 & 3,4 & 20,4 & 5,3 & 37,4 & 6,5 & 14,2 & 4,3 & 27,1 & 5,7 \\
1986 & 9,1 & 3,4 & 19,3 & 3,5 & 33,8 & 6,3 & 13,9 & 3,4 & 26,5 & 4,5 \\
1987 & 9,6 & 3,5 & 18,7 & 4,1 & 34,5 & 5,1 & 14,0 & 3,8 & 25,8 & 4,5 \\
1988 & 8,9 & 3,5 & 19,6 & 4,3 & 33,1 & 6,0 & 14,2 & 3,9 & 24,1 & 4,7 \\
1989 & 9,4 & 4,2 & 21,2 & 5,3 & 33,9 & 6,1 & 15,3 & 4,8 & 25,3 & 5,4 \\
1990 & 8,9 & 3,6 & 22,6 & 5,2 & 33,9 & 6,0 & 15,8 & 4,4 & 26,1 & 5,2 \\
1991 & 9,2 & 3,6 & 21,8 & 5,8 & 33,8 & 5,6 & 15,5 & 4,7 & 27,1 & 5,6 \\
1992 & 8,3 & 3,3 & 23,8 & 4,9 & 33,1 & 5,1 & 16,0 & 4,1 & 31,4 & 5,0 \\
1993 & 8,7 & 3,7 & 22,5 & 5,6 & 33,9 & 5,7 & 15,5 & 4,7 & 28,1 & 5,7 \\
1994 & 7,1 & 3,2 & 23,0 & 5,1 & 35,8 & 5,2 & 15,0 & 4,2 & 29,1 & 5,1 \\
1995 & 7,3 & 3,2 & 20,4 & 5,2 & 31,4 & 5,7 & 13,8 & 4,2 & 25,7 & 5,4 \\
\hline
\end{tabular}

Fuente/Source:OPS.Sistema de Información Técnica

*Tasa/Rate x 100.000 habitantes

M:Masculino/Male

F:Femenino/Female 
Cuadro 6.c - Tasa* de mortalidad de adolescentes y jóvenes por suicidio, según edad y sexo México 1980-1995

Table 6.c - Mortality rate among adolescents and young adults due to suicide, by age and sex Mexico 1980-1995

\begin{tabular}{cccccccccccc}
\hline Edad & \multicolumn{3}{c}{ 10 a 14 años } & \multicolumn{2}{c}{ 15 a 19 años } & \multicolumn{2}{c}{ 20 a 24 años } & \multicolumn{2}{c}{10 a 19 años } & \multicolumn{2}{c}{15 a 24 años } \\
\hline Años & M & F & M & F & M & F & M & F & M & F \\
\hline 1980 & 0,5 & 0,1 & 3,2 & 1,3 & 4,4 & 1,4 & 1,7 & 0,6 & 3,7 & 1,4 \\
1981 & 0,4 & 0,2 & 2,7 & 1,8 & 6,8 & 1,6 & 1,5 & 0,9 & 4,5 & 1,7 \\
1982 & 0,5 & 0,2 & 2,6 & 1,6 & 5,8 & 1,8 & 1,4 & 0,8 & 4,0 & 1,7 \\
1983 & 0,5 & 0,1 & 2,4 & 1,0 & 4,6 & 1,2 & 1,3 & 0,5 & 3,4 & 1,1 \\
1984 & 0,4 & 0,1 & 2,2 & 0,7 & 3,3 & 0,9 & 1,2 & 0,4 & 2,6 & 0,7 \\
1985 & 0,7 & 0,3 & 2,8 & 1,4 & 6,9 & 1,3 & 1,7 & 0,8 & 4,5 & 1,3 \\
1986 & 0,7 & 0,2 & 3,3 & 1,5 & 6,7 & 1,3 & 1,9 & 0,8 & 5,0 & 1,3 \\
1987 & 0,6 & 0,3 & 3,5 & 1,4 & 6,2 & 1,3 & 2,0 & 0,8 & 4,7 & 1,3 \\
1988 & 0,8 & 0,3 & 3,1 & 1,2 & 6,5 & 1,5 & 1,9 & 0,7 & 4,4 & 1,3 \\
1989 & 0,7 & 0,3 & 3,9 & 1,2 & 6,6 & 0,9 & 2,3 & 0,7 & 4,8 & 1,0 \\
1990 & 0,7 & 0,2 & 4,0 & 1,4 & 6,2 & 1,0 & 2,4 & 0,8 & 4,7 & 1,2 \\
1991 & 0,8 & 0,3 & 3,8 & 0,9 & 8,1 & 1,5 & 2,3 & 0,6 & 5,8 & 1,2 \\
1992 & 0,8 & 0,4 & 4,5 & 1,6 & 7,0 & 1,0 & 2,6 & 1,0 & 6,3 & 1,3 \\
1993 & 0,8 & 0,4 & 4,5 & 1,4 & 7,3 & 1,2 & 2,6 & 0,9 & 5,9 & 1,3 \\
1994 & 0,8 & 0,5 & 4,4 & 1,8 & 8,9 & 1,6 & 2,6 & 1,1 & 6,5 & 1,7 \\
1995 & 0,9 & 0,5 & 5,1 & 2,3 & 10,3 & 1,7 & 3,0 & 1,4 & 7,6 & 2,0 \\
\hline
\end{tabular}

Fuente/Source:OPS. Sistema de Información Técnica

*Tasa/Rate x 100.000 habitantes

M:Masculino/Male

F:Femenino/Female

Cuadro 6.d - Tasa* de mortalidad de adolescentes y jóvenes por homicidio, según edad y sexo México 1980-1995

Table 6.d - Mortality rate among adolescents and young adults due to homicide, by age and sex Mexico 1980-1995

\begin{tabular}{ccccccccccc}
\hline Edad & \multicolumn{1}{c}{10 a 14 años } & \multicolumn{2}{c}{15 a 19 años } & \multicolumn{2}{c}{20 a 24 años } & \multicolumn{2}{c}{10 a 19 años } & \multicolumn{2}{c}{15 a 24 años } \\
\hline Años & M & F & M & F & M & F & M & F & M & F \\
\hline 1980 & 3,4 & 1,1 & 24,1 & 2,8 & 58,1 & 4,4 & 12,7 & 1,9 & 39,2 & 3,5 \\
1981 & 3,9 & 1,2 & 26,8 & 3,1 & 60,8 & 4,1 & 14,3 & 2,1 & 42,0 & 3,6 \\
1982 & 3,2 & 1,3 & 25,0 & 2,4 & 62,5 & 3,9 & 13,0 & 1,8 & 41,7 & 3,1 \\
1983 & 3,2 & 1,0 & 23,3 & 2,5 & 58,0 & 4,4 & 12,3 & 1,7 & 38,8 & 3,4 \\
1984 & 3,3 & 1,1 & 22,5 & 2,2 & 52,1 & 4,0 & 12,0 & 1,6 & 34,4 & 2,9 \\
1985 & 3,5 & 0,8 & 27,6 & 3,0 & 62,2 & 4,7 & 14,6 & 1,8 & 41,6 & 3,6 \\
1986 & 3,7 & 1,3 & 30,2 & 3,6 & 66,1 & 4,1 & 16,1 & 2,3 & 47,5 & 3,6 \\
1987 & 4,1 & 1,4 & 28,7 & 2,6 & 63,2 & 3,9 & 15,8 & 2,0 & 44,2 & 3,2 \\
1988 & 3,8 & 1,5 & 27,2 & 3,5 & 57,3 & 3,9 & 15,2 & 2,5 & 38,3 & 3,5 \\
1989 & 3,9 & 1,4 & 27,2 & 3,0 & 55,1 & 4,5 & 15,5 & 2,2 & 37,4 & 3,5 \\
1990 & 3,8 & 1,6 & 24,7 & 3,2 & 48,8 & 4,1 & 14,3 & 2,4 & 33,6 & 3,4 \\
1991 & 4,2 & 1,4 & 27,3 & 3,9 & 53,7 & 3,7 & 15,8 & 2,7 & 39,2 & 3,8 \\
1992 & 4,0 & 1,2 & 29,7 & 3,7 & 59,6 & 4,8 & 16,8 & 2,4 & 48,9 & 4,2 \\
1993 & 4,0 & 1,1 & 29,1 & 3,4 & 53,3 & 3,8 & 16,4 & 2,2 & 41,0 & 3,6 \\
1994 & 3,4 & 1,5 & 27,8 & 3,4 & 56,7 & 3,6 & 15,5 & 2,4 & 41,5 & 3,5 \\
1995 & 3,3 & 1,4 & 26,5 & 3,4 & 51,6 & 4,0 & 14,8 & 2,4 & 38,6 & 3,7 \\
\hline
\end{tabular}

Fuente/Source:OPS. Sistema de Información Técnica

*Tasa/Rate x 100.000 habitantes

M:Masculino/Male

F:Femenino/Female 
La mortalidad por accidentes de tráfico es el componente principal de la mortalidad por causas externas en la población total hasta el año 1983. Desde el año 1984 en adelante, la tasa de mortalidad por homicidio ocupa el primer lugar. Esto se debe a un descenso mayor de la mortalidad por accidentes de tráfico (30\% de descenso) respecto a la mortalidad por homicidio ( $14 \%$ de descenso), en la población total en el período estudiado.

En el grupo de adolescentes de 10 a 14 años la mortalidad por accidentes de tráfico es la primera causa de mortalidad por causas externas y duplica a la mortalidad por homicidios en 1995 (Cuadro 6.b). En cambio, en el grupo de hombres de 15 a 24 años las tasas de mortalidad por homicidio sobrepasan a las tasas de mortalidad por accidentes de tráfico, desde el año 1982 (Cuadro 6.d).

La mortalidad por accidentes de tráfico ha disminuido progresivamente, en todos los grupos de adolescentes, durante el período estudiado. En cambio, la mortalidad por homicidio ha aumentado en un $10 \%$ entre los hombres y $21 \%$ en las mujeres de 15 a 19 años. En el grupo de 20 a 24 años hay un descenso de $11 \%$ entre los hombres y $9 \%$ entre las mujeres (Cuadro 6.d).

Las tasas de mortalidad por suicidio han aumentado en la población total de 1,5 por 100.000 en 1980 a 3,2 por 100.000 en 1995 (Cuadro 1.9 en Anexo). En los jóvenes varones de 15 a 24 años ha aumentado en un $105 \%$. Entre las mujeres jóvenes las tasas de mortalidad por suicidio también sufren aumento, mismo en sus valores significativamente menores (Cuadro 6.c).

En suma, la disminución de la mortalidad por causas externas en adolescentes y jóvenes se debe principalmente a una disminución de la mortalidad por accidentes de tráfico. La mortalidad por homicidio es la primera causa de mortalidad por causas externas entre los hombres de 15 a 24 años y su tendencia és decreciente, asi como entre los adolescentes de 15 a 19 años. Entre las mujeres se observa una estabilizacíon en ambos los grupos etários.

\section{Países del Area Andina}

\section{COLOMBIA}

Colombia es el país estudiado con mayores tasas de mortalidad por causas externas en la población total y en adolescentes y jóvenes (Cuadros 1.3 a 1.5 en Anexo y 7.a). Entre los años extremos del período estudiado,1981 a 1992, hay un aumento de un $29 \%$ en las tasas de mortalidad por causas externas para la población total, de un 94,6 a 122,1 por 100.000 , seguido de un descenso de 19\% entre los años 1992 y 1995.

En la población adolescente y joven la tasa de mortalidad por causa externa se ha mantenido estable en la población de 10 a 14 años (alrededor de 31 por 100.000 en los hombres y 12,5 por 100.000 en las mujeres), entre 1984 y 1995 (Cuadro 7.a). En cambio, en los grupos de 15 a 19 y 20 a 24 años, de ambos sexos, ha habido un aumento dramático en las tasas en el mismo período. Hay un aumento de un $146 \%$ en las tasas de los hombres de 15 a 19 años (de 88,9 a 218,8 por 100.000 ), y de un $52 \%$ en las mujeres (de 21,2 a 32,2 por 100.000) de igual edad. En el grupo de 20 a 24 años hay un aumento de un $67 \%$ en los hombres (de 212,6 a 355,7 por 100.000 ) y de un $22 \%$ en las mujeres (de 27,7 a 33,9 por 100.000) El grupo de hombres de 20 a 24 años presenta las tasas de mortalidad mas altas por causas externas entre adolescentes y jóvenes, sin embargo, el grupo de hombres de 15 a 19 años es el que ha experimentado un mayor aumento de las tasas entre 1984 y 1995. En 1995 el cuociente de mortalidad por causas externas hombres/mujeres entre 10 y 14 años es de 2,5/1; entre 15 a 19 años es de 7 / 1 ; y entre 20 y 24 años es de $11 / 1$.

El componente mas importante de la mortalidad por causas externas en Colombia, tanto en la población total como en adolescentes y jóvenes es la mortalidad por homicidio. En la población total la tasa de homicidio asciende en $177 \%$ entre 1984 y 1992, descendiendo posteriormente en un $32 \%$ hasta el año 1995, llegando a tasas de 60,8 por 100.000 (Cuadro 1.12 en Anexo). 
Cuadro 7.a - Tasa* de mortalidad de adolescentes y jóvenes por causas externas, según edad y sexo - Colombia 1984-1995

Table 7.a - Mortality rate among adolescents and young adults due to external causes, by age and sex-Colombia 1984-1995

\begin{tabular}{ccccccccccc}
\hline Edad & \multicolumn{3}{c}{10 a 14 años } & \multicolumn{2}{c}{15 a 19 años } & \multicolumn{2}{c}{20 a 24 años } & \multicolumn{2}{c}{10 a 19 años } & \multicolumn{2}{c}{15 a 24 años } \\
\hline Años & M & F & M & F & M & F & M & F & M & F \\
\hline 1984 & 30,7 & 10,9 & 88,9 & 21,2 & 212,6 & 27,7 & 59,7 & 16,1 & 146,3 & 24,2 \\
1985 & 30,0 & 11,6 & 111,3 & 24,5 & 254,3 & 31,3 & 70,6 & 18,1 & 178,3 & 27,7 \\
1986 & 29,5 & 10,5 & 118,3 & 23,7 & 269,4 & 29,7 & 73,7 & 17,1 & 190,1 & 26,6 \\
1987 & 28,6 & 12,1 & 127,6 & 25,2 & 274,8 & 30,6 & 77,3 & 18,6 & 198,6 & 27,8 \\
1988 & 29,5 & 13,2 & 141,9 & 24,6 & 303,5 & 28,6 & 84,0 & 18,7 & 221,0 & 26,6 \\
1989 & 29,2 & 11,4 & 162,3 & 27,7 & 313,9 & 30,2 & 92,9 & 19,2 & 237,2 & 29,0 \\
1990 & 28,3 & 10,9 & 183,3 & 28,1 & 329,0 & 33,9 & 101,9 & 19,1 & 255,5 & 31,0 \\
1991 & 32,9 & 11,8 & 216,7 & 30,6 & 395,9 & 34,7 & 120,2 & 20,8 & 305,1 & 32,6 \\
1992 & 34,9 & 12,4 & 213,8 & 30,7 & 390,8 & 36,1 & 120,2 & 21,2 & 300,1 & 33,4 \\
1993 & 33,2 & 11,6 & 221,2 & 30,3 & 400,1 & 36,6 & 123,6 & 20,6 & 307,0 & 33,4 \\
1994 & 32,6 & 11,7 & 229,8 & 32,0 & 392,8 & 34,4 & 128,1 & 21,6 & 307,0 & 33,2 \\
1995 & 31,0 & 12,5 & 218,8 & 32,2 & 355,7 & 33,9 & 122,5 & 22,1 & 283,2 & 33,0 \\
\hline
\end{tabular}

Fuente/Source:OPS. Sistema de Información Técnica

*Tasa/Rate x 100.000 habitantes

M: Masculino/Male

F: Femenino/Female

Cuadro 7.b - Tasa* de mortalidad de adolescentes y jóvenes por accidentes de vehículo a motor, según edad y sexo - Colombia 1984-1995

Table 7.b - Mortality rate among adolescents and young adults due to traffic accidents, by age and sex-Colombia 1984-1995

\begin{tabular}{ccccccccccc}
\hline Edad & \multicolumn{2}{c}{ 10 a 14 años } & \multicolumn{2}{c}{ 15 a 19 años } & \multicolumn{2}{c}{20 a 24 años } & \multicolumn{2}{c}{10 a 19 años } & \multicolumn{2}{c}{15 a 24 años } \\
\hline Años & M & F & M & F & M & F & M & F & M & F \\
\hline 1984 & 9,5 & 3,2 & 16,0 & 4,2 & 31,7 & 6,8 & 12,7 & 3,7 & 23,3 & 5,4 \\
1985 & 8,6 & 3,0 & 14,9 & 4,8 & 30,3 & 6,7 & 11,8 & 3,9 & 22,1 & 5,7 \\
1986 & 7,4 & 2,8 & 13,9 & 4,0 & 24,8 & 5,8 & 10,6 & 3,4 & 19,1 & 4,9 \\
1987 & 7,2 & 2,7 & 14,1 & 4,3 & 25,8 & 5,2 & 10.6 & 3,5 & 19,7 & 4,7 \\
1988 & 7,6 & 2,6 & 13,1 & 4,7 & 24,6 & 4,7 & 10,3 & 3,7 & 18,7 & 4,7 \\
1989 & 6,9 & 2,9 & 12,5 & 4,9 & 23,9 & 4,6 & 9,6 & 3,8 & 18,2 & 4,7 \\
1990 & 6.9 & 2,7 & 13,1 & 5,0 & 23,3 & 5,7 & 9,8 & 3,8 & 18,1 & 5,4 \\
1991 & 8,4 & 3,0 & 13,7 & 3,9 & 23,5 & 5,4 & 10,9 & 3,4 & 18,5 & 4,7 \\
1992 & 8,9 & 3,2 & 15,4 & 5,0 & 28,2 & 6,2 & 12,0 & 4,1 & 21,7 & 5,6 \\
1993 & 7,9 & 2,5 & 16,8 & 4,7 & 29,5 & 5,6 & 12,2 & 3,5 & 22,9 & 5,1 \\
1994 & 8,0 & 3,2 & 19,8 & 4,7 & 33,9 & 7,0 & 13,7 & 4,0 & 26,5 & 5,8 \\
1995 & 8,4 & 3,4 & 21,2 & 7,2 & 37,3 & 7,6 & 14,6 & 5,3 & 28,8 & 7,4 \\
\hline
\end{tabular}

Fuente/Source:OPS. Sistema de Información Técnica

*Tasa/Rate 100.000 habitantes

M:Masculino/Male

F:Femenino/Female

Las tasas de homicidios en todos los grupos de adolescentes y jóvenes sufren un dramático incremento, paralelo a las causas externas, entre los años estudiados (Cuadro 7.d). El grupo de hombres de 10 a 14 años triplica las tasas de mortalidad por homicidio en el período estudiado, alcanzando tasas de 9,4 por 100.000 en 1995. Las mujeres de igual edad duplican las tasas para llegar a valores de 3,5 por 100.000 en 1995. El grupo de hombres de 15 a 19 años es el grupo que sufre el mayor aumento de la tasa de mortalidad por homicidio en el grupo estudiado, llegando el año 1995 a una tasa de 155,5 por 100.000 , casi 5 veces mayor que el año 1984. Las mujeres de igual 
Cuadro 7.c - Tasa* de mortalidad de adolescentes y jóvenes por suicidio, según edad y sexo Colombia 1984-1995

Table 7.c - Mortality rate among adolescents and young adults due to suicide, by age and sex Colombia 1984-1995

\begin{tabular}{ccccccccccc}
\hline Edad & \multicolumn{2}{c}{10 a 14 años } & \multicolumn{2}{c}{15 a 19 años } & \multicolumn{2}{c}{20 a 24 años } & \multicolumn{2}{c}{10 a 19 años } & \multicolumn{2}{c}{15 a 24 años } \\
\hline Años & M & F & M & F & M & F & M & F & M & F \\
\hline 1984 & 0,7 & 0,8 & 4,6 & 4,0 & 12,8 & 2,9 & 2,7 & 2,4 & 8,4 & 3,5 \\
1985 & 0,6 & 0,5 & 5,2 & 3,7 & 9,6 & 3,0 & 2,9 & 2,1 & 7,3 & 3,4 \\
1986 & 0,5 & 0,5 & 4,6 & 4,1 & 10,2 & 2,7 & 2,6 & 2,3 & 7,3 & 3,4 \\
1987 & 0,8 & 0,5 & 5,7 & 3,4 & 11,2 & 3,2 & 3,2 & 1,9 & 8,3 & 3,3 \\
1988 & 0,7 & 0,5 & 4,4 & 2,4 & 9,1 & 2,3 & 2,5 & 1,4 & 6,7 & 2,4 \\
1989 & 0,5 & 0,7 & 3,5 & 2,4 & 7,0 & 1,9 & 1,9 & 1,5 & 5,2 & 2,2 \\
1990 & 0,6 & 0,4 & 4,3 & 2,8 & 7,8 & 2,4 & 2,3 & 1,5 & 6,0 & 2,6 \\
1991 & 1,2 & 0,5 & 4,8 & 3,0 & 10,5 & 2,5 & 2,9 & 1,7 & 7,6 & 2,7 \\
1992 & 0,8 & 0,8 & 5,1 & 3,3 & 9,8 & 2,7 & 2,9 & 2,0 & 7,4 & 3,0 \\
1993 & 1,0 & 0,4 & 5,4 & 3,0 & 9,8 & 2,5 & 3,1 & 1,7 & 7,5 & 2,7 \\
1994 & 0,6 & 0,7 & 6,1 & 3,8 & 10,9 & 3,2 & 3,3 & 2,2 & 8,3 & 3,5 \\
1995 & 0,7 & 0,5 & 4,8 & 3,3 & 9,8 & 2,9 & 2,7 & 1,8 & 7,2 & 3,1 \\
\hline
\end{tabular}

Fuente/Source:OPS. Sistema de Información Técnica

*Tasa/Rate x 100.000 habitantes

M:Masculino/Male

F:Femenino/Female

Cuadro 7.d - Tasa* de mortalidad de adolescentes y jóvenes por homicidio, según edad y sexo Colombia 1984-1995

Table 7.d - Mortality rate among adolescents and young adults due to homicide, by age and sex Colombia 1984-1995

\begin{tabular}{ccccccccccc}
\hline Edad & \multicolumn{3}{c}{ 10 a 14 años } & \multicolumn{2}{c}{ 15 a 19 años } & \multicolumn{2}{c}{20 a 24 años } & \multicolumn{2}{c}{10 a 19 años } & \multicolumn{2}{c}{15 a 24 años } \\
\hline Años & M & F & M & F & M & F & M & F & M & F \\
\hline 1984 & 3,4 & 1,2 & 34,9 & 5,7 & 105,8 & 7,4 & 19,1 & 3,5 & 67,8 & 6,5 \\
1985 & 4,2 & 1,3 & 48,8 & 5,1 & 136,0 & 8,5 & 26,5 & 3,2 & 89,7 & 6,7 \\
1986 & 4,3 & 1,0 & 56,8 & 6,5 & 157,2 & 10,1 & 30,4 & 3,7 & 104,5 & 8,2 \\
1987 & 4,3 & 1,0 & 63,8 & 7,2 & 166,2 & 11,0 & 33,6 & 4,1 & 113,2 & 9,0 \\
1988 & 6,1 & 2,0 & 86,0 & 8,2 & 198,0 & 13,2 & 44,8 & 5,0 & 140,8 & 10,7 \\
1989 & 6,3 & 2,0 & 109,0 & 11,4 & 227,9 & 14,6 & 55,4 & 6,5 & 167,8 & 13,0 \\
1990 & 8,9 & 2,5 & 138,5 & 13,8 & 255,9 & 18,6 & 70,5 & 7,9 & 196,7 & 16,2 \\
1991 & 10,3 & 3,5 & 171,2 & 16,8 & 315,9 & 20,2 & 86,7 & 9,8 & 242,6 & 18,5 \\
1992 & 12,0 & 3,9 & 166,8 & 15,5 & 316,4 & 19,8 & 85,8 & 9,5 & 239,7 & 17,7 \\
1993 & 11,7 & 3,0 & 173,7 & 16,0 & 322,2 & 22,3 & 89,6 & 9,3 & 245,0 & 19,0 \\
1994 & 12,0 & 3,0 & 180,4 & 16,8 & 309,1 & 16,8 & 93,6 & 9,7 & 241,3 & 16,8 \\
1995 & 9,4 & 3,5 & 155,5 & 13,1 & 249,4 & 15,2 & 80,5 & 8,2 & 199,6 & 14,1 \\
\hline
\end{tabular}

Fuente/Source:OPS. Sistema de Información Técnica

*Tasa/Rate x 100.000 habitantes

M:Masculino/Male

F: Femenino/Female

edad triplican las tasas en igual período, llegando a valores de 13,1 por 100.000 el año 1995. El grupo de hombres de 20 a 24 años presenta las tasas mas altas de mortalidad por homicidio ( 249,4 por 100.000 en 1995 ), duplicando las tasas desde 1984. Las tasas de mujeres de igual edad tambien se duplican en el mismo período, llegando en 1995 a tasas próximas al grupo de mujeres de 15 a 19 años. El cuocientes de mortalidad por homicidio hombre/mujer es de $3 / 1$ en el grupo de 10 a 14; 12/1 entre 15 a 19 y 16/1 entre 20 y 24 años. El descenso observado en las tasas de homicidio en la población total entre 1992 y 1995 se refleja en los adolescentes y en los jovenes de ambos sexos en todos los grupos etários.

La mortalidad por accidentes de tráfico 
es el segundo componente de la mortalidad por causas externas en Colombia. En la población total las tasas decrecen entre 1984 y 1989 y aumentan en un 33\% después, entre 1989 y 1995, sin producir cambios significativos entre las fechas extremas del período estudiado. Se observa la misma tendencia en los adolescentes y jóvenes, con un $56 \%$ de aumento entre los hombres de 15 a 24 años entre 1989 y 1995, lo que determina un incremento de un $24 \%$ entre los años extremos (Cuadro 7.b). La tasa de mortalidad por homicidios triplica la tasa de mortalidad por accidentes de tráfico en el grupo de hombres de 15 a 24 años en 1984. En cambio, en 1995 es 7 veces mayor.

Las tasas de mortalidad por suicidio son decrecientes en la población total desde 1984 hasta 1989, fecha en que se observa una leve tendencia al incremento hasta 1995. La misma tendencia se observa en jóvenes de 20 a 24 años. En los grupos de 10 a 19 años las tasas se han mantenido estables, con valores de 4,8 por 100.000 en hombres de 15 a 19 años y 9,8 por 100.000 en hombres de 20 a 24 años (Cuadro 7.c).

En conclusión, Colombia es el país estudiado con tasas mas altas de mortalidad por causas externas y homicidios en la población total y adolescentes y jóvenes. El dramático incremento de la mortalidad por causas externas se debe al aumento de los homicidios. El grupo con tasas mas altas de mortalidad por homicidios, entre los adolescentes y jóvenes, es el de hombres de 20 a 24 años. El grupo que ha sufrido el mayor incremento en las tasas entre 1984 y 1995 es el de hombres de 15 a 19 años.

\section{VENEZUELA}

La mortalidad por causas externas en la población total disminuye entre los años 1980 y 1988 en un $24 \%$ y posteriormente aumenta progresivamente entre 1988 y 1994 hasta alcanzar valores de 123,2 por 100.000 en el sexo masculino. No hay cambios significativos entre las fechas extremas del período estudiado (1980-1994) (Cuadros 1.3 a 1.5 en Anexo). Esta misma tendencia se observa en todos los grupos de adolescentes y jóvenes (Cuadro 8.a). En el grupo de 15 a 24 años , a pesar del descenso en las tasas durante el primer período, hay un aumento en la mortalidad por causas externas de un $15 \%$ entre las fechas extremas estudiadas. Este aumento está determinado por un incremento de un $75 \%$ de las tasas entre los años 1988 y 1994.

El principal determinante de la mortalidad por causas externas en la población total de Venezuela es la mortalidad por accidentes de tráfico, que se ha reducido en un 35\% entre los años 1980 y 1994, a pesar que entre los años 1990 y 1994 existe una leve tendencia al ascenso. La misma tendencia se observa en todos los grupos de adolescentes (Cuadro 8.b).

La mortalidad por homicidios en la población total de Venezuela sufre un descenso de un 31\% entre los años 1980 y 1987. Posteriormente hay un brusco ascenso de un $107 \%$ entre los años 1987 y 1994, llegando a tasas de 16,8 por 100.000 (Cuadro 1.12 en Anexo). Entre los dos años extremos de la serie hay un aumento de un $44 \%$ de las tasas en la población total.

Esta tendencia se observa también en los grupos de adolescentes y jóvenes. Entre los años 1987 y 1994 hay un aumento de un $216 \%$ en la tasa de mortalidad por homicidio entre los adolescentes varones de 15 a 19 años, con tasas de 54,9 por 100.000 al final del período estudiado. Entre los hombres de 20 a 24 años hay un $150 \%$ de aumento entre las mismas fechas, llegando a tasas 85,4 por 100.000 en 1994. Entre los dos años extremos de la serie hay un aumento de un $65 \%$ de las tasas en el grupo de 15 a 24 años. La relación entre las tasas de mortalidad por homicidio hombre/mujer de 15 a 24 años es de 20/ 1 (Cuadro 8.d).

La tasa de mortalidad por suicidio se ha mantenido estacionaria, con leves oscilaciones, en el período estudiado, tanto en población total como en adolescentes y jóvenes (Cuadros 1.9 en Anexo y 8.c).

En resumen, la tendencia de la mortalidad por causas externas en los adolescentes y jóvenes de Venezuela está determinada por 
Cuadro 8.a - Tasa* de mortalidad de adolescentes y jóvenes por causas externas, según edad y sexo - Venezuela 1980-1994

Table 8.a - Mortality rate among adolescents and young adults due to external causes, by age and sex-Venezuela 1980-1994

\begin{tabular}{ccccccccccc}
\hline Edad & \multicolumn{1}{c}{10 a 14 años } & \multicolumn{2}{c}{15 a 19 años } & \multicolumn{2}{c}{20 a 24 años } & \multicolumn{2}{c}{10 a 19 años } & \multicolumn{2}{c}{15 a 24 años } \\
\hline Años & M & F & M & F & M & F & M & F & M & F \\
\hline 1980 & 37,0 & 13,8 & 133,8 & 25,3 & 239,4 & 27,3 & 83,4 & 19,3 & 181,6 & 26,2 \\
1981 & 34,0 & 15,2 & 120,6 & 30,3 & 219,0 & 29,3 & 75,9 & 22,5 & 165,5 & 29,8 \\
1982 & 33,9 & 12,6 & 113,7 & 27,9 & 228,3 & 26,9 & 72,7 & 20,1 & 166,6 & 27,5 \\
1983 & 32,8 & 12,9 & 111,8 & 23,5 & 214,6 & 26,4 & 71,4 & 18,1 & 159,9 & 24,9 \\
1985 & 29,2 & 12,3 & 86,6 & 17,8 & 153,2 & 23,3 & 57,1 & 15,0 & 119,0 & 20,6 \\
1986 & 29,9 & 12,1 & 94,6 & 20,0 & 154,5 & 22,5 & 61,1 & 15,9 & 123,6 & 21,2 \\
1987 & 30,2 & 11,6 & 94,8 & 19,2 & 150,1 & 21,0 & 60,9 & 15,2 & 121,6 & 20,1 \\
1988 & 30,1 & 11,7 & 92,0 & 21,5 & 149,6 & 19,8 & 59,2 & 16,3 & 119,2 & 20,5 \\
1989 & 28,7 & 10,1 & 112,4 & 21,1 & 161,0 & 20,5 & 67,6 & 15,2 & 134,6 & 20,6 \\
1990 & 29,2 & 10,8 & 107,7 & 23,0 & 161,7 & 23,5 & 65,5 & 16,4 & 132,1 & 22,9 \\
1992 & 31,9 & 12,6 & 139,9 & 20,4 & 199,8 & 25,6 & 82,3 & 16,2 & 165,8 & 22,5 \\
1993 & 31,4 & 12,5 & 153,6 & 22,0 & 222,3 & 25,0 & 89,0 & 17,0 & 183,0 & 23,0 \\
1994 & 33,1 & 11,4 & 173,2 & 24,9 & 250,6 & 25,9 & 99,7 & 17,8 & 208,8 & 25,3 \\
\hline
\end{tabular}

Fuente/Source:OPS. Sistema de Información Técnica

*Tasa/Rate x 100.000 habitantes

M:Masculino/Male

F:Femenino/Female

Cuadro 8.b - Tasa* de mortalidad de adolescentes y jóvenes por accidentes de vehículo a motor, según edad y sexo - Venezuela 1980-1994

Table 8.b - Mortality rate among adolescents and young adults due to traffic accidents, by age and sex-Venezuela 1980-1994

\begin{tabular}{|c|c|c|c|c|c|c|c|c|c|c|}
\hline \multirow{2}{*}{$\frac{\text { Edad }}{\text { Años }}$} & \multicolumn{2}{|c|}{10 a 14 años } & \multicolumn{2}{|c|}{15 a 19 años } & \multicolumn{2}{|c|}{20 a 24 años } & \multicolumn{2}{|c|}{10 a 19 años } & \multicolumn{2}{|c|}{15 a 24 años } \\
\hline & M & $\mathrm{F}$ & $M$ & $\mathrm{~F}$ & M & $\mathrm{F}$ & M & $\mathrm{F}$ & M & $F$ \\
\hline 1980 & 16,3 & 6,4 & 59,3 & 12,8 & 114,3 & 13,2 & 36,9 & 9,5 & 84,2 & 13,0 \\
\hline 1981 & 16,0 & 6,4 & 53,7 & 14,9 & 105,9 & 15,4 & 34,3 & 10,5 & 77,5 & 15,1 \\
\hline 1982 & 13,6 & 7,2 & 54,2 & 12,5 & 103,7 & 13,5 & 33,4 & 9,8 & 77,1 & 12,9 \\
\hline 1983 & 15,0 & 6,4 & 49,5 & 11,1 & 90,2 & 12,1 & 31,9 & 8,7 & 68,5 & 11,6 \\
\hline 1985 & 10,9 & 6,3 & 30,7 & 8,4 & 59,1 & 9,7 & 20,5 & 7,4 & 44,5 & 9,1 \\
\hline 1986 & 11,3 & 5,9 & 36,4 & 10,1 & 64,8 & 10,2 & 23,4 & 7,9 & 50,2 & 10,2 \\
\hline 1987 & 12,3 & 5,1 & 34,0 & 10,6 & 58,7 & 10,0 & 22,6 & 7,7 & 46,0 & 10,3 \\
\hline 1988 & 12,6 & 4,2 & 30,1 & 10,0 & 56,6 & 10,4 & 20,8 & 6,9 & 42,7 & 10,1 \\
\hline 1989 & 10,1 & 4,5 & 28,9 & 9,9 & 43,0 & 9,6 & 18,9 & 7,0 & 35,4 & 9,7 \\
\hline 1990 & 9,2 & 5,0 & 25,6 & 10,7 & 49,6 & 10,4 & 16,8 & 7,7 & 36,7 & 10,4 \\
\hline 1992 & 12,5 & 6,4 & 31,7 & 8,3 & 52,4 & 12,2 & 21,5 & 7,3 & 40,9 & 10,0 \\
\hline 1993 & 13,3 & 6,9 & 30,5 & 10,3 & 55,2 & 12,4 & 21,4 & 8,5 & 41,5 & 11,1 \\
\hline 1994 & 11,1 & 5,2 & 31,1 & 10,4 & 58,9 & 10,9 & 20,6 & 7,7 & 43,9 & 10,7 \\
\hline
\end{tabular}

Fuente/Source:OPS. Sistema de Información Técnica

*Tasa/Rate x 100.000 habitantes

M:Masculino/Male

F: Femenino/Female

la mortalidad por accidentes de tráfico y los homicidios. Ambas causas sufren tendencias similares, con disminución en un primer período (1980-1989) y aumento a partir de
1989. El aumento es especialmente notorio en las tasas de mortalidad por homicidio en los jóvenes varones de 15 a 24 años. 
Cuadro 8.c - Tasa* de mortalidad de adolescentes y jóvenes por suicidio, según edad y sexo Venezuela 1980-1994

Table 8.c - Mortality rate among adolescents and young adults due to suicide, by age and sex Venezuela 1980-1994

\begin{tabular}{lllllllllll}
\hline Edad & \multicolumn{3}{c}{ 10 a 14 años } & \multicolumn{2}{c}{ 15 a 19 años } & \multicolumn{2}{c}{20 a 24 años } & \multicolumn{2}{c}{10 a 19 años } & \multicolumn{2}{c}{15 a 24 años } \\
\hline Años & M & F & M & F & M & F & M & F & M & F \\
\hline 1980 & 0,6 & 0,8 & 6,7 & 3,1 & 13,2 & 3,7 & 3,5 & 1,9 & 9,6 & 3,3 \\
1981 & 0,8 & 0,6 & 5,2 & 4,4 & 13,2 & 3,3 & 2,9 & 2,4 & 8,9 & 3,9 \\
1982 & 0,5 & 0,4 & 5,1 & 3,8 & 12,8 & 3,3 & 2,8 & 2,1 & 8,7 & 3,6 \\
1983 & 0,5 & 0,7 & 5,9 & 4,4 & 13,2 & 3,0 & 3,2 & 2,6 & 9,3 & 3,8 \\
1985 & 0,4 & 0,3 & 4,4 & 3,0 & 9,8 & 3,7 & 2,4 & 1,6 & 7,0 & 3,3 \\
1986 & 0,6 & 1,1 & 5,4 & 2,5 & 14,4 & 3,2 & 2,9 & 1,8 & 9,7 & 2,9 \\
1987 & 0,3 & 0,5 & 7,6 & 1,9 & 11,8 & 2,5 & 3,8 & 1,2 & 9,7 & 2,2 \\
1988 & 1,2 & 1,0 & 6,9 & 4,0 & 12,1 & 1,9 & 3,9 & 2,4 & 9,4 & 2,9 \\
1989 & 1,6 & 0,9 & 7,7 & 3,2 & 14,0 & 2,9 & 4,4 & 2,0 & 10,6 & 3,0 \\
1990 & 1,0 & 1,3 & 8,7 & 3,1 & 13,6 & 3,9 & 4,6 & 2,1 & 10,9 & 3,5 \\
1992 & 1,5 & 0,4 & 8,2 & 2,7 & 15,3 & 2,4 & 4,6 & 1,5 & 11,4 & 2,5 \\
1993 & 1,2 & 0,3 & 7,4 & 3,0 & 15,1 & 2,0 & 4,2 & 1,5 & 10,9 & 2,5 \\
1994 & 2,0 & 0,8 & 7,1 & 3,8 & 16,6 & 3,9 & 4,5 & 2,2 & 11,5 & 3,8 \\
\hline
\end{tabular}

Fuente/Source:OPS. Sistema de Información Técnica

*Tasa/Rate x 100.000 habitantes

M:Masculino/Male

F:Femenino/Female

Cuadro 8.d - Tasa* de mortalidad de adolescentes y jóvenes por homicidio, según edad y sexo Venezuela 1980-1994

Table 8.d - Mortality rate among adolescents and young adults due to homicide, by age and sex Venezuela 1980-1994

\begin{tabular}{ccccccccccc}
\hline Edad & 10 a 14 años & \multicolumn{2}{c}{15 a 19 años } & \multicolumn{2}{c}{20 a 24 años } & \multicolumn{2}{c}{10 a 19 años } & \multicolumn{2}{c}{15 a 24 años } \\
\hline Años & M & F & M & F & M & F & M & F & M & $F$ \\
\hline 1980 & 1,9 & 0,5 & 25,5 & 2,8 & 61,3 & 3,4 & 13,2 & 1,6 & 41,7 & 3,1 \\
1981 & 1,0 & 0,6 & 18,9 & 2,4 & 45,3 & 2,4 & 9,7 & 1,5 & 31,0 & 2,4 \\
1982 & 1,8 & 0,9 & 22,4 & 2,5 & 55,1 & 3,4 & 11,8 & 1,6 & 37,5 & 2,9 \\
1983 & 1,7 & 0,4 & 24,8 & 2,3 & 58,7 & 3,9 & 13,0 & 1,4 & 40,6 & 3,1 \\
1985 & 1,9 & 0,2 & 18,8 & 1,9 & 38,9 & 3,0 & 10,1 & 1,0 & 28,6 & 2,4 \\
1986 & 1,0 & 0,3 & 19,2 & 1,6 & 32,0 & 3,5 & 9,8 & 0,9 & 25,4 & 2,5 \\
1987 & 0,9 & 0,5 & 17,4 & 1,6 & 34,2 & 2,0 & 8,8 & 1,0 & 25,6 & 1,8 \\
1988 & 2,6 & 0,8 & 21,4 & 1,7 & 38,6 & 2,6 & 11,4 & 1,2 & 29,6 & 2,1 \\
1989 & 3,5 & 0,6 & 40,0 & 2,9 & 61,2 & 3,6 & 20,5 & 1,7 & 49,8 & 3,2 \\
1990 & 3,5 & 0,9 & 41,0 & 3,1 & 62,2 & 3,2 & 20,8 & 1,9 & 50,5 & 3,1 \\
1992 & 2,7 & 1,0 & 43,3 & 2,6 & 64,2 & 3,6 & 21,7 & 1,7 & 52,4 & 3,0 \\
1993 & 3,4 & 0,8 & 52,3 & 2,9 & 72,2 & 3,8 & 26,5 & 1,8 & 60,7 & 3,2 \\
1994 & 4,0 & 1,1 & 54,9 & 2,7 & 85,4 & 4,4 & 28,1 & 1,8 & 68,9 & 3,5 \\
\hline
\end{tabular}

Fuente/Source:OPS. Sistema de Información Técnica

*Tasa/Rate x 100.000 habitantes

M:Masculino/Male

F:Femenino/Female

\section{ECUADOR}

La tasa de mortalidad por causas externas en la población total de Ecuador ha disminuido progresivamente en un $10 \%$ en el decenio estudiado (1980-1990) y practicamente estable de 1990 a 1996 (Cuadros 1.3 a 1.5 en Anexo). Similar tendencia se observa en los adolescentes y jóvenes de ambos sexos, con un $17 \%$ de descenso entre las mismas fechas en el grupo de hombres de 15 a 24 años, llegando en 1990 a tasas de 100,8 por 100.000 (Cuadro 9.a). Entre 1990 y 1996 se observa aumento en esa respecti- 
Cuadro 9.a -Tasa* de mortalidad de adolescentes y jóvenes por causas externas, según edad y sexo - Ecuador 1980-1996

Table 9.a - Mortality rate among adolescents and young adults due to external causes, by age and sex-Ecuador 1980-1996

\begin{tabular}{ccccccccccr}
\hline Edad & \multicolumn{1}{c}{10 a 14 años } & \multicolumn{2}{c}{15 a 19 años } & \multicolumn{2}{c}{20 a 24 años } & \multicolumn{2}{c}{10 a 19 años } & \multicolumn{2}{c}{15 a 24 años } \\
\hline Años & M & F & M & F & M & F & M & F & M & F \\
\hline 1980 & 42,5 & 19,8 & 92,6 & 32,7 & 156,1 & 25,6 & 65,6 & 25,7 & 121,8 & 29,4 \\
1981 & 42,7 & 20,8 & 88,1 & 32,9 & 153,4 & 37,0 & 63,8 & 26,4 & 118,1 & 34,8 \\
1982 & 34,2 & 20,7 & 81,5 & 32,6 & 149,9 & 30,1 & 56,1 & 26,2 & 112,9 & 31,4 \\
1983 & 39,7 & 18,8 & 84,8 & 29,2 & 150,5 & 31,0 & 60,7 & 23,6 & 115,0 & 30,0 \\
1984 & 33,3 & 16,6 & 80,9 & 28,8 & 139,5 & 27,6 & 55,6 & 22,3 & 103,7 & 27,2 \\
1985 & 29,9 & 15,4 & 71,6 & 22,7 & 137,6 & 26,3 & 49,4 & 18,8 & 97,8 & 23,4 \\
1986 & 31,4 & 13,1 & 71,7 & 25,0 & 134,9 & 27,3 & 50,3 & 18,7 & 96,9 & 25 \\
1987 & 33,8 & 18,7 & 71,9 & 24,4 & 123,3 & 20,4 & 51,7 & 21,3 & 95,7 & 22,5 \\
1988 & 35,6 & 14,5 & 76,8 & 22,8 & 135,2 & 20,3 & 55,0 & 18,4 & 99,8 & 20,8 \\
1989 & 38,3 & 13,7 & 69,0 & 26,2 & 120,5 & 23,9 & 52,7 & 19,6 & 89,3 & 24,1 \\
1990 & 35,0 & 16,6 & 77,2 & 23,4 & 136,3 & 25,8 & 54,9 & 19,8 & 100,8 & 23,6 \\
1991 & 31,9 & 14,5 & 85,5 & 27,1 & 126,7 & 26,3 & 57,2 & 20,5 & 104,8 & 26,7 \\
1992 & 36,6 & 15,7 & 80,6 & 25,1 & 145,2 & 20,2 & 57,5 & 20,2 & 110,9 & 22,8 \\
1993 & 30,1 & 16,4 & 77,9 & 32,4 & 134,3 & 28,7 & 52,9 & 24,0 & 104,3 & 30,7 \\
1994 & 27,3 & 12,4 & 69,8 & 23,9 & 127,6 & 26,7 & 47,7 & 17,9 & 96,9 & 25,2 \\
1995 & 27,8 & 12,1 & 83,5 & 28,7 & 140,5 & 23,5 & 54,6 & 20,1 & 110,3 & 26,2 \\
1996 & 27,8 & 12,8 & 85,2 & 28,3 & 135,4 & 25,1 & 55,6 & 20,3 & 108,9 & 26,8 \\
\hline
\end{tabular}

Fuente/Source:OPS. Sistema de Información Técnica

*Tasa/Rate 100.000 habitantes

M:Masculino/Male

F: Femenino/Female

Cuadro 9.b - Tasa* de mortalidad de adolescentes y jóvenes por accidentes de vehículo a motor, según edad y sexo - Ecuador 1980-1996

Table 9.b - Mortality rate among adolescents and young adults due to traffic accidents, by age and sex-Ecuador 1980-1996

\begin{tabular}{ccccccccccc}
\hline Edad & \multicolumn{2}{c}{10 a 14 años } & \multicolumn{2}{c}{15 a 19 años } & \multicolumn{2}{c}{20 a 24 años } & \multicolumn{2}{c}{10 a 19 años } & \multicolumn{2}{c}{15 a 24 años } \\
\hline Años & M & F & M & F & M & F & M & F & M & F \\
\hline 1980 & 17,3 & 6,7 & 35,0 & 7,6 & 51,0 & 7,2 & 25,4 & 7,1 & 42,3 & 7,4 \\
1981 & 19,1 & 9,1 & 29,1 & 10,1 & 54,0 & 10,5 & 23,8 & 9,6 & 40,5 & 10,3 \\
1982 & 12,3 & 6,0 & 23,5 & 6,0 & 49,9 & 8,1 & 17,5 & 6,0 & 35,6 & 7,0 \\
1983 & 10,7 & 4,4 & 19,0 & 6,5 & 29,1 & 5,1 & 14,6 & 5,4 & 23,7 & 5,8 \\
1984 & 11,2 & 7,6 & 24,2 & 7,8 & 38,8 & 6,9 & 17,3 & 7,7 & 29,7 & 7,1 \\
1985 & 8,3 & 4,2 & 20,6 & 6,8 & 39,0 & 6,9 & 14,0 & 5,4 & 27,9 & 6,6 \\
1986 & 9,6 & 4,2 & 17,3 & 5,6 & 36,1 & 6,7 & 13,2 & 4,9 & 24,9 & 5,9 \\
1987 & 11,3 & 5,7 & 18,1 & 5,3 & 32,5 & 5,4 & 14,5 & 5,5 & 24,8 & 5,3 \\
1988 & 13,1 & 4,7 & 22,6 & 5,1 & 40,3 & 5,2 & 17,6 & 4,9 & 29,6 & 5,0 \\
1989 & 15,6 & 5,6 & 20,8 & 5,6 & 30,5 & 6,8 & 18,1 & 5,6 & 24,3 & 5,9 \\
1990 & 12,9 & 5,0 & 23,5 & 4,7 & 36,7 & 9,1 & 17,9 & 4,9 & 28,6 & 6,5 \\
1991 & 11,8 & 4,6 & 26,1 & 7,6 & 38,3 & 5,2 & 18,6 & 6,0 & 31,8 & 6,5 \\
1992 & 14,0 & 5,5 & 20,9 & 5,4 & 41,6 & 6,3 & 17,3 & 5,4 & 30,6 & 5,8 \\
1993 & 12,8 & 5,7 & 24,9 & 8,1 & 37,5 & 5,9 & 18,6 & 6,9 & 30,8 & 7,1 \\
1994 & 7,4 & 4,3 & 15,3 & 4,3 & 29,1 & 6,7 & 11,2 & 4,3 & 21,8 & 5,5 \\
1995 & 9,3 & 2,7 & 19,8 & 4,9 & 27,3 & 5,8 & 14,3 & 3,8 & 23,3 & 5,3 \\
1996 & 7,6 & 3,3 & 18,4 & 4,8 & 27,2 & 4,5 & 12,8 & 4,0 & 22,5 & 4,6 \\
\hline
\end{tabular}

Fuente/Source:OPS. Sistema de Información Técnica

*Tasa/Rate x 100.000 habitantes

M:Masculino/Male

F:Femenino/Female 
Cuadro 9.c - Tasa* de mortalidad de adolescentes y jóvenes por suicidio, según edad y sexo Ecuador 1980-1996

Table 9.c - Mortality rate among adolescents and young adults due to suicide, by age and sex Ecuador 1980-1996

\begin{tabular}{|c|c|c|c|c|c|c|c|c|c|c|}
\hline \multirow{2}{*}{$\frac{\text { Edad }}{\text { Años }}$} & \multicolumn{2}{|c|}{10 a 14 años } & \multicolumn{2}{|c|}{15 a 19 años } & \multicolumn{2}{|c|}{20 a 24 años } & \multicolumn{2}{|c|}{10 a 19 años } & \multicolumn{2}{|c|}{15 a 24 años } \\
\hline & M & $\mathrm{F}$ & $M$ & $\mathrm{~F}$ & $M$ & $F$ & M & $\mathrm{F}$ & M & $\mathrm{F}$ \\
\hline 1980 & 0,4 & 0,8 & 7,4 & 9,5 & 9,8 & 6,1 & 3,6 & 4,8 & 8,5 & 7,9 \\
\hline 1981 & 0,2 & 1,2 & 5,8 & 10,8 & 13,8 & 9,2 & 2,8 & 5,6 & 9,5 & 10,1 \\
\hline 1982 & 0,2 & 1,7 & 7,0 & 12,5 & 9,5 & 10,2 & 3,3 & 6,7 & 8,1 & 11,4 \\
\hline 1983 & 0,6 & 1,3 & 7,0 & 11,3 & 11,4 & 8,1 & 3,5 & 6,0 & 9,0 & 9,8 \\
\hline 1984 & 0,9 & 0,6 & 7,2 & 10,9 & 13,7 & 9,9 & 3,8 & 5,4 & 9,8 & 10,0 \\
\hline 1985 & 1,2 & 0,7 & 8,6 & 6,8 & 14,3 & 6,0 & 4,7 & 3,6 & 10,7 & 6,1 \\
\hline 1986 & 1,0 & 1,1 & 6,2 & 7,8 & 12,5 & 8,1 & 3,5 & 4,2 & 8,8 & 7,6 \\
\hline 1987 & 1,3 & 0,7 & 7,0 & 9,7 & 9,0 & 6,5 & 4,0 & 4,9 & 8,0 & 8,2 \\
\hline 1988 & 0,7 & 0,8 & 7,8 & 8,0 & 13,9 & 6,8 & 4,0 & 4,2 & 10,2 & 7,1 \\
\hline 1989 & 1,1 & 0,8 & 3,6 & 9,7 & 11,8 & 7,8 & 2,3 & 5,0 & 7,1 & 8,5 \\
\hline 1990 & 0,9 & 0,8 & 6,4 & 8,9 & 12,3 & 7,2 & 3,5 & 4,6 & 8,8 & 7,8 \\
\hline 1991 & 0,5 & 1,4 & 6,9 & 11,3 & 13,2 & 10,6 & 3,5 & 6,1 & 9,8 & 11,0 \\
\hline 1992 & 1,1 & 2,0 & 7,3 & 11,4 & 13,6 & 5,1 & 4,0 & 6,5 & 10,2 & 8,5 \\
\hline 1993 & 1,1 & 1,4 & 6,3 & 12,2 & 12,5 & 11,3 & 3,5 & 6,6 & 9,2 & 11,8 \\
\hline 1994 & 1,8 & 1,8 & 6,5 & 9,0 & 9,3 & 7,7 & 4,0 & 5,3 & 7,8 & 8,4 \\
\hline 1995 & 0,9 & 1,5 & 7,0 & 10,7 & 12,3 & 5,3 & 3,8 & 6,0 & 9,5 & 8,2 \\
\hline 1996 & 1,0 & 2,6 & 7,8 & 10,2 & 13,2 & 6,8 & 4,3 & 6,3 & 10,4 & 8,6 \\
\hline
\end{tabular}

Fuente/Source:OPS. Sistema de Información Técnica

*Tasa/Rate x 100.000 habitantes

M:Masculino/Male

F:Femenino/Female

Cuadro 9.d - Tasa* de mortalidad de adolescentes y jóvenes por homicidio, según edad y sexo Ecuador 1980-1996

Table 9.d - Mortality rate among adolescents and young adults due to homicide, by age and sex Ecuador 1980-1996

\begin{tabular}{ccccccccccc}
\hline Edad & \multicolumn{3}{c}{ 10 a 14 años } & \multicolumn{2}{c}{15 a 19 años } & \multicolumn{2}{c}{20 a 24 años } & \multicolumn{2}{c}{10 a 19 años } & \multicolumn{2}{c}{15 a 24 años } \\
\hline Años & M & F & M & F & M & F & M & $F$ & $M$ & F \\
\hline 1980 & 0,6 & 0,8 & 10,9 & 1,2 & 25,3 & 0,8 & 5,3 & 1,0 & 17,5 & 1,0 \\
1981 & 1,0 & 0,0 & 10,1 & 1,4 & 27,0 & 3,8 & 5,2 & 0,6 & 17,8 & 2,5 \\
1982 & 0,4 & 0,8 & 11,3 & 2,5 & 32,4 & 2,9 & 5,5 & 1,6 & 21,0 & 2,7 \\
1983 & 0,7 & 0,6 & 11,6 & 1,3 & 37,6 & 3,0 & 5,8 & 0,9 & 23,5 & 2,1 \\
1984 & 1,1 & 0,4 & 12,7 & 1,7 & 33,5 & 1,7 & 6,5 & 1,0 & 21,4 & 1,6 \\
1985 & 1,2 & 0,5 & 11,8 & 2,3 & 33,4 & 4,1 & 6,2 & 1,3 & 24,5 & 3,0 \\
1986 & 2,6 & 0,7 & 15,6 & 2,6 & 35,6 & 5,1 & 8,7 & 1,6 & 23,8 & 3,6 \\
1987 & 1,2 & 1,4 & 12,7 & 1,8 & 37,4 & 2,9 & 6,6 & 1,6 & 24,2 & 2,3 \\
1988 & 2,5 & 1,0 & 11,9 & 1,5 & 37,1 & 2,8 & 6,9 & 1,3 & 27,4 & 2,0 \\
1989 & 2,9 & 1,2 & 14,7 & 2,6 & 34,0 & 3,2 & 8,4 & 1,8 & 22,7 & 2,8 \\
1990 & 1,7 & 0,6 & 17,0 & 1,1 & 37,7 & 2,9 & 8,9 & 0,9 & 25,7 & 1,9 \\
1991 & 2,0 & 1,0 & 16,4 & 2,3 & 36,3 & 2,4 & 8,8 & 1,6 & 25,8 & 2,4 \\
1992 & 1,8 & 0,8 & 20,3 & 1,6 & 39,8 & 2,5 & 10,6 & 1,2 & 29,4 & 2,0 \\
1993 & 1,8 & 0,5 & 16,0 & 3,7 & 43,1 & 2,7 & 8,6 & 2,0 & 28,7 & 3,2 \\
1994 & 2,5 & 0,8 & 16,0 & 1,7 & 38,8 & 3,4 & 9,0 & 1,2 & 26,7 & 2,5 \\
1995 & 1,3 & 0,8 & 21,7 & 3,9 & 47,1 & 4,2 & 11,1 & 2,3 & 33,7 & 4,0 \\
1996 & 2,6 & 0,2 & 22,9 & 3,0 & 47,9 & 3,2 & 12,4 & 1,6 & 34,7 & 3,1 \\
\hline
\end{tabular}

Fuente/Source:OPS. Sistema de Información Técnica

*Tasa/Rate x 100.000 habitantes

M:Masculino/Male

F:Femenino/Female 
va tasa, llegando a 108,9 por 100.000 . Solamente en el grupo de 10 a 14 años las tasas por causas externas disminuyen, siendo de 27,8 para los hombres y 12,8 para las mujeres en 1996.

La determinante principal de la mortalidad por causas externas en la población total y en los adolescentes y jóvenes de Ecuador son los accidentes de tráfico. En la población total la tasa de mortalidad por accidentes de tráfico ha disminuido progresivamente en un $45 \%$ en el período de 1980 a 1996 y, en un 47\% entre los jóvenes hombres de 15 a 24 años (Cuadros 1.6 en Anexo y 9.b).

En cambio, la tasa de mortalidad por homicidio en Ecuador ha sufrido un aumento progresivo en la población total de un $119 \%$ y un aumento de un $98 \%$ entre los jóvenes varones de 15 a 24 años, entre las mismas fechas. En 1980 la tasa de mortalidad por accidentes de tráfico en los hombres de 20 a 24 años duplica la tasa de mortalidad por homicidios, siendo de un 51 y 25,3, respectivamente (Cuadros 9.b y 9.d). En 1996 la tasa de mortalidad por homicidios en el mismo grupo (47,9 por 100.000) supera la tasa de mortalidad por accidentes de tráfico (27,2 por 100.000). El cuociente de mortalidad por homicidio entre hombres y mujeres es de $11 / 1$ en la población de 15 a 24 años (Cuadro 9.d).

Las tasas de mortalidad por suicidio se han mantenido estables en el período de 1980 a 1990, tanto en la población total como en adolescentes y jóvenes de Ecuador (Cuadros 1.11 en Anexo y 9.c). Pero, entre 1990 y 1996 se observa aumento en ambos sexos y en todos los grupos etários, aún cuando sus valores son menores que los de homicidios y accidentes.

En resumen, en Ecuador la tasa de mortalidad por causas externas ha disminuido en los adolescentes y jóvenes a raíz de una disminución de los accidentes de tráfico. La tasa de mortalidad por homicidios está aumentando progresivamente en este grupo etáreo y ha superado la tasa de mortalidad por accidentes de tráfico en los jóvenes de 20 a 24 años.

\section{Países del Cono Sur}

\section{ARGENTINA}

Las tasas de mortalidad por causas externas se han mantenido estables en la población total con pequeñas oscilaciones y disminución de un 9\% entre las fechas extremas del período estudiado (1980-1996) (Cuadros 1.3 a 1.5 en Anexo). Las tasas han aumentado levemente (7\%) entre los jóvenes varones de 15 a 24 años, llegando a 83,8 por 100.000 en el año 1996. En cambio, entre las mujeres de 15 a 24 años las tasas han disminuido en un $10 \%$, alcanzando cifras de 21,6 por 100.000 en 1996. También hay tendencia a la disminución de $21 \%$ en los hombres y $33 \%$ en las mujeres, en el grupo de 10 a 14 años (Cuadro 10.a).

La principal determinante de la mortalidad por causas externas en la población total de Argentina son los accidentes de tráfico, seguido cercanamente por los suicidios y después, homicidios. En la población total la tasa de mortalidad por accidentes de tráfico disminuye en un $21 \%$ en el período estudiado, los suicidios disminuyen en un $10 \%$ y los homicidios aumentan en un 37\% (Cuadros 1.6, 1.9 y 1.12 en Anexo). En la población adolescente el determinante principal de la tasa de mortalidad por causas externas son los accidentes de tráfico, seguido por homicidios (Cuadros 10.b y 10.d).

La tasa de mortalidad por accidentes de tráfico se ha mantenido estable en los adolescentes hombres y hay una tendencia a una leve disminución en los grupos de mujeres de 10 a 19 años (Cuadro 10.b).

La tendencia de la mortalidad por suicidio difiere de la tendencia sufrida por la población total. En el grupo de adolescentes varones el suicidio ha aumentado en el período estudiado, especialmente en el grupo de 10 a 14 años, de 0,4 a 0,7 por 100.000 entre 1980 y 1996. En los hombres de 15 a 24 años se observa un aumento de las tasas de un $44 \%$ en igual período, llegando a tasas de 8,8 por 100.000 en 1996. La tasa de mortalidad por suicidio es levemente decre- 
Cuadro 10.a - Tasa* de mortalidad de adolescentes y jóvenes por causas externas, según edad y sexo - Argentina 1980-1996

Table 10.a - Mortality rate among adolescents and young adults due to external causes, by age and sex-Argentina 1980-1996

\begin{tabular}{|c|c|c|c|c|c|c|c|c|c|c|}
\hline \multirow{2}{*}{$\begin{array}{l}\text { Edad } \\
\text { Años }\end{array}$} & \multicolumn{2}{|c|}{10 a 14 años } & \multicolumn{2}{|c|}{15 a 19 años } & \multicolumn{2}{|c|}{20 a 24 años } & \multicolumn{2}{|c|}{10 a 19 años } & \multicolumn{2}{|c|}{15 a 24 años } \\
\hline & M & $\mathrm{F}$ & M & $\mathrm{F}$ & M & $\mathrm{F}$ & M & $F$ & M & $\mathrm{F}$ \\
\hline 1980 & 25,1 & 12,6 & 65,1 & 24,1 & 92,9 & 23,6 & 44,4 & 18,2 & 78,5 & 23,9 \\
\hline 1981 & 29,3 & 11,4 & 63,2 & 22,2 & 95,6 & 22,4 & 45,6 & 16,6 & 78,9 & 22,3 \\
\hline 1982 & 23,4 & 9,2 & 60,2 & 19,2 & 84,7 & 20,0 & 41,0 & 14,0 & 72,1 & 19,6 \\
\hline 1983 & 26,7 & 10,6 & 61,6 & 17,2 & 85,6 & 20,1 & 43,3 & 13,7 & 73,3 & 18,6 \\
\hline 1984 & 25.4 & 9.5 & 61,5 & 19,3 & 83,4 & 19,2 & 42,5 & 14,1 & 75,8 & 19,4 \\
\hline 1985 & 22,5 & 9,2 & 57,7 & 15,4 & 82,3 & 17,7 & 39,0 & 12,1 & 69,6 & 16,7 \\
\hline 1986 & 24,0 & 10,4 & 65,0 & 18,1 & 86,5 & 22,8 & 43,0 & 14,0 & 75,5 & 21,2 \\
\hline 1987 & 21,0 & 8,6 & 64,0 & 21,2 & 83,2 & 20,1 & 40,7 & 14,4 & 73,2 & 20,7 \\
\hline 1988 & 21,5 & 8,6 & 66,4 & 21,1 & 85,4 & 19,0 & 41,9 & 14,3 & 75,8 & 20,3 \\
\hline 1989 & 22.0 & 9,3 & 70,6 & 19,8 & 91,3 & 19,1 & 44,0 & 14,1 & 80,8 & 19,7 \\
\hline 1990 & 21,8 & 8,3 & 58,4 & 16,2 & 85,7 & 19,2 & 38,5 & 11,9 & 71,8 & 17,9 \\
\hline 1991 & 21,8 & 8,7 & 60,0 & 19,1 & 81,6 & 17,8 & 39,5 & 13,5 & 70,9 & 18,9 \\
\hline 1992 & 23,4 & 11,2 & 67,9 & 19,8 & 91,7 & 18,1 & 44,5 & 15,3 & 80,0 & 19,5 \\
\hline 1993 & 23,8 & 10,0 & 68,2 & 19,7 & 96,5 & 21,0 & 45,4 & 14,7 & 82,4 & 20,8 \\
\hline 1994 & 26,7 & 9,3 & 74,5 & 21,0 & 103,5 & 24,5 & 50,5 & 15,1 & 87,7 & 22.6 \\
\hline 1995 & 20,6 & 9,7 & 69,8 & 20,4 & 99,5 & 21,9 & 45,4 & 15,1 & 83,3 & 21,1 \\
\hline 1996 & 19,9 & 8,4 & 67,5 & 22,2 & 102,6 & 20,9 & 44,0 & 15,4 & 83,8 & 21,6 \\
\hline
\end{tabular}

Fuente/Source:OPS. Sistema de Información Técnica

*Tasa/Rate x 100.000 habitantes

M:Masculino/Male

F:Femenino/Female

Cuadro 10.b - Tasa* de mortalidad de adolescentes y jóvenes por accidentes de vehículo a motor, según edad y sexo - Argentina 1980-1996

Table 10.b - Mortality rate among adolescents and young adults due to traffic accidents, by age and sex-Argentina 1980-1996

\begin{tabular}{ccccccccccc}
\hline Edad & \multicolumn{1}{c}{10 a 14 años } & \multicolumn{2}{c}{15 a 19 años } & \multicolumn{2}{c}{20 a 24 años } & \multicolumn{2}{c}{10 a 19 años } & \multicolumn{2}{c}{15 a 24 años } \\
\hline Años & M & F & M & F & M & F & M & F & M & F \\
\hline 1980 & 5,6 & 4,0 & 16,8 & 6,3 & 24,8 & 6,2 & 11,0 & 5,1 & 20,7 & 6,2 \\
1981 & 6,9 & 3,5 & 16,5 & 6,1 & 23,9 & 4,8 & 11,5 & 4,8 & 20,1 & 5,5 \\
1982 & 5,3 & 2,8 & 15,4 & 4,8 & 23,3 & 5,8 & 10,1 & 3,7 & 19,2 & 5,3 \\
1983 & 6,8 & 3,3 & 15,2 & 3,7 & 24,0 & 4,8 & 10,8 & 3,5 & 19,5 & 4,3 \\
1984 & 6,4 & 3,2 & 14,8 & 4,3 & 19,5 & 5,7 & 10,3 & 3,8 & 17,0 & 5,0 \\
1985 & 5,8 & 2,5 & 8,9 & 3,7 & 17,1 & 3,9 & 7,3 & 3,0 & 12,9 & 3,8 \\
1986 & 6,3 & 3,1 & 11,5 & 4,1 & 19,3 & 6,0 & 8,7 & 3,5 & 15,3 & 5,2 \\
1987 & 5,6 & 2,3 & 11,6 & 4,8 & 16,3 & 4,6 & 8,4 & 3,5 & 13,9 & 4,7 \\
1988 & 5,8 & 2,4 & 12,2 & 3,8 & 17,1 & 4,4 & 8,7 & 3,0 & 14,6 & 4,1 \\
1989 & 5,1 & 2,8 & 13,4 & 3,3 & 19,0 & 4,6 & 8,9 & 3,0 & 16,1 & 3,9 \\
1990 & 5,7 & 2,0 & 10,2 & 4,1 & 17,2 & 4,4 & 7,8 & 3,0 & 13,6 & 4,3 \\
1991 & 5,2 & 2,1 & 12,7 & 5,5 & 16,8 & 5,2 & 8,7 & 3,7 & 14,8 & 5,5 \\
1992 & 5,9 & 2,7 & 15,1 & 4,4 & 22,4 & 5,0 & 10,3 & 3,5 & 18,7 & 4,8 \\
1993 & 5,4 & 2,3 & 15,8 & 5,4 & 26,3 & 6,6 & 10,4 & 3,8 & 20,9 & 6,1 \\
1994 & 7,0 & 2,6 & 15,6 & 6,1 & 30,3 & 8,2 & 11,3 & 4,4 & 22,3 & 7,0 \\
1995 & 4,9 & 2,8 & 16,9 & 6,3 & 26,4 & 6,6 & 10,9 & 4,6 & 21,2 & 6,5 \\
1996 & 5,5 & 2,9 & 16,5 & 6,2 & 26,5 & 5,7 & 11,1 & 4,6 & 21,1 & 6,0 \\
\hline
\end{tabular}

Fuente/Source:OPS. Sistema de Información Técnica

*Tasa/Rate x 100.000 habitantes

M:Masculino/Male

F:Femenino/Female 
Cuadro 10.c - Tasa* de mortalidad de adolescentes y jóvenes por suicidio, según edad y sexo Argentina 1980-1996

Table 10.c - Mortality rate among adolescents and young adults due to suicide, by age and sex Argentina 1980-1996

\begin{tabular}{ccccccccccc}
\hline Edad & \multicolumn{1}{c}{ 10 a 14 años } & \multicolumn{2}{c}{15 a 19 años } & \multicolumn{2}{c}{20 a 24 años } & \multicolumn{2}{c}{10 a 19 años } & \multicolumn{2}{c}{15 a 24 años } \\
\hline Años & M & F & M & F & M & F & M & F & M & F \\
\hline 1980 & 0,4 & 1,5 & 4,5 & 4,9 & 7,9 & 4,8 & 2,4 & 3,2 & 6,1 & 4,9 \\
1981 & 0,8 & 1,0 & 4,2 & 4,3 & 11,1 & 4,4 & 2,4 & 2,6 & 7,5 & 4,4 \\
1982 & 0,7 & 0,5 & 4,5 & 4,0 & 8,8 & 4,2 & 2,5 & 2,2 & 6,6 & 4,1 \\
1983 & 0,4 & 0,5 & 5,0 & 3,5 & 7,6 & 3,4 & 2,6 & 1,9 & 6,3 & 3,4 \\
1984 & 1,0 & 0,2 & 3,6 & 3,3 & 7,7 & 2,4 & 2,2 & 1,7 & 5,5 & 2,9 \\
1985 & 1,1 & 0,8 & 4,3 & 2,7 & 8,8 & 3,1 & 2,6 & 1,7 & 6,5 & 2,9 \\
1986 & 0,7 & 0,5 & 4,6 & 3,9 & 10,8 & 4,5 & 2,5 & 2,1 & 7,6 & 4,4 \\
1987 & 1,0 & 0,9 & 4,5 & 4,4 & 10,1 & 3,1 & 2,6 & 2,5 & 7,2 & 3,8 \\
1988 & 1,2 & 0,6 & 5,3 & 4,8 & 11,6 & 3,3 & 3,1 & 2,5 & 8,3 & 4,1 \\
1989 & 0,9 & 0,6 & 4,6 & 3,4 & 10,1 & 2,8 & 2,6 & 1,9 & 7,2 & 3,2 \\
1990 & 1,2 & 0,8 & 4,7 & 3,4 & 8,7 & 3,6 & 2,8 & 2,0 & 6,6 & 3,5 \\
1991 & 1,6 & 0,5 & 4,9 & 2,7 & 8,4 & 1,8 & 3,1 & 1,5 & 6,6 & 2,3 \\
1992 & 1,1 & 0,8 & 6,4 & 2,7 & 11,7 & 3,0 & 3,6 & 1,7 & 8,9 & 2,9 \\
1993 & 1,4 & 0,9 & 5,9 & 2,3 & 10,6 & 2,8 & 3,6 & 1,6 & 8,2 & 2,6 \\
1994 & 1,5 & 0,5 & 6,9 & 3,8 & 9,8 & 2,7 & 4,2 & 2,2 & 8,3 & 3,3 \\
1995 & 0,8 & 1,1 & 6,7 & 3,6 & 11,6 & 2,9 & 3,8 & 2,4 & 8,9 & 3,3 \\
1996 & 0,7 & 0,6 & 7,1 & 4,0 & 10,7 & 2,6 & 4,0 & 2,3 & 8,8 & 3,4 \\
\hline
\end{tabular}

Fuente/Source:OPS. Sistema de Información Técnica

*Tasa/Rate x 100.000 habitantes

M:Masculino/Male

F:Femenino/Female

Cuadro 10.d - Tasa* de mortalidad de adolescentes y jóvenes por homicidio, según edad y sexo Argentina 1981-1996

Table 10.d - Mortality rate among adolescents and young adults due to homicide, by age and sex Argentina 1981-1996

\begin{tabular}{ccccccccccc}
\hline Edad & \multicolumn{2}{c}{10 a 14 años } & \multicolumn{2}{c}{15 a 19 años } & \multicolumn{2}{c}{20 a 24 años } & \multicolumn{2}{c}{10 a 19 años } & \multicolumn{2}{c}{15 a 24 años } \\
\hline Años & M & F & M & F & M & F & M & F & M & F \\
\hline 1981 & 0,8 & 1,0 & 4,2 & 4,3 & 11,1 & 4,4 & 2,4 & 2,6 & 7,5 & 4,4 \\
1982 & 0,7 & 0,5 & 4,5 & 4,0 & 8,8 & 4,2 & 2,5 & 2,2 & 6,6 & 4,1 \\
1983 & 0,6 & 0,6 & 5,3 & 1,4 & 11,1 & 1,8 & 2,8 & 1,0 & 8,1 & 1,6 \\
1984 & 0,7 & 0,4 & 9,3 & 1,8 & 13,9 & 2,9 & 4,8 & 1,1 & 11,5 & 2,4 \\
1985 & 0,9 & 0,5 & 9,9 & 1,7 & 19,6 & 2,5 & 5,1 & 1,1 & 14,6 & 2,1 \\
1986 & 2,0 & 0,8 & 12,0 & 2,3 & 20,0 & 2,8 & 6,6 & 1,5 & 15,8 & 2,7 \\
1987 & 1,8 & 0,7 & 13,6 & 2,8 & 18,3 & 3,3 & 7,2 & 1,7 & 15,8 & 3,1 \\
1988 & 1,5 & 0,6 & 14,8 & 2,8 & 19,4 & 2,7 & 7,5 & 1,6 & 17,0 & 2,8 \\
1989 & 1,2 & 0,7 & 16,0 & 2,9 & 22,5 & 2,8 & 7,9 & 1,7 & 19,2 & 2,9 \\
1990 & 1,4 & 0,5 & 11,4 & 1,3 & 19,0 & 1,7 & 6,0 & 0,9 & 15,1 & 1,5 \\
1991 & 0,9 & 0,4 & 8,6 & 1,5 & 12,9 & 1,7 & 4,4 & 0,9 & 10,7 & 1,6 \\
1992 & 1,6 & 1,1 & 8,9 & 2,2 & 13,4 & 1,4 & 5,1 & 1,6 & 11,1 & 1,9 \\
1993 & 1,2 & 0,5 & 8,8 & 1,5 & 12,8 & 1,9 & 4,9 & 1,0 & 10,8 & 1,8 \\
1994 & 1,6 & 0,7 & 8,3 & 1,3 & 13,1 & 2,4 & 5,0 & 1,0 & 10,5 & 1,8 \\
1995 & 0,9 & 0,6 & 7,7 & 1,1 & 11,6 & 1,7 & 4,3 & 0,9 & 9,5 & 1,4 \\
1996 & 1,1 & 0,6 & 9,6 & 1,7 & 12,6 & 2,3 & 5,4 & 1,2 & 11,0 & 2,0 \\
\hline
\end{tabular}

Fuente/Source:OPS. Sistema de Información Técnica

*Tasa/Rate x 100.000 habitantes

M:Masculino/Male

F:Femenino/Female 
ciente en las mujeres de igual edad (Cuadro 10.c).

Las tasas de mortalidad por homicidio han aumentado en todos los grupos de adolescentes y jóvenes varones, duplicándose en los hombres de 15 a 19 años entre 1981 y 1996. En las mujeres de 15 a 24 años las tasas decrecen en un $48 \%$ en el mismo período (Cuadro 10.d).

En resumen, el componente fundamental de la mortalidad por causas externas en la población total de Argentina son los accidentes de tráfico. Sin embargo, en los adolescentes y jóvenes hombres de 15 a 24 años el aumento en la mortalidad por causas externas se debe principalmente al aumento de las tasas de mortalidad por homicidios y suicidios en este grupo etáreo.

\section{CHILE}

La mortalidad por causas externas en la población total disminuye en un 19\% en el período estudiado (1980-1994) (Cuadros 1.3 a 1.5 en Anexo). También hay una disminución progresiva de la mortalidad por cau- sas externas en los adolescentes de 10 a 14 años (34\% de disminución en varones y $29 \%$ en mujeres) y en el grupo de 15 a 24 años fue de un $12 \%$ para los hombres y $32 \%$ para las mujeres (Cuadro 11.a).

La causa determinante de la mortalidad por causas externas en la población total de Chile es la mortalidad por accidentes de tráfico, que sufre una tendencia al descenso entre 1980 y 1988 y luego aumenta en un $71 \%$ entre el año 1988 y 1994, sin variaciones significativas entre las fechas extremas del período estudiado. La tasa de mortalidad por suicidio es el segundo determinante de la mortalidad por causas externas en la población total de Chile, manteniendo tasas estables, cercanas a 5 por 100.000. Las tasas de mortalidad por homicidio son bajas y estables, alrededor de 3 por 100.000 en la población total (Cuadros 1.6, 1.9 y 1.12 en Anexo).

La mortalidad por accidentes de tráfico en adolescentes y jóvenes sufre una tendencia similar a la población total, con un primer período de descenso de un $2 \%$ en varones de 15 a 24 años y un aumento de un

Cuadro 11.a - Tasa* de mortalidad de adolescentes y jóvenes por causas externas, según edad y sexo - Chile 1980-1994

Table 11.a - Mortality rate among adolescents and young adults due to external causes, by age and sex - Chile 1980-1994

\begin{tabular}{lcrrrrrrrrr}
\hline Edad & \multicolumn{2}{c}{10 a 14 años } & \multicolumn{2}{c}{15 a 19 años } & \multicolumn{2}{c}{20 a 24 años } & \multicolumn{2}{c}{10 a 19 años } & \multicolumn{2}{c}{15 a 24 años } \\
\hline Años & M & F & M & F & M & F & M & F & M & F \\
\hline 1980 & 28,8 & 13,3 & 74,1 & 19,8 & 132,1 & 24,4 & 51,4 & 16,5 & 101,1 & 21,9 \\
1981 & 28,6 & 13,0 & 71,5 & 18,6 & 125,9 & 23,1 & 50,2 & 15,8 & 118,3 & 20,7 \\
1982 & 28,3 & 8,9 & 66,2 & 17,5 & 116,3 & 19,6 & 47,4 & 13,2 & 110,3 & 18,5 \\
1983 & 26,3 & 10,5 & 66,7 & 18,7 & 127,5 & 19,9 & 46,7 & 14,6 & 96,3 & 19,3 \\
1984 & 22,6 & 9,1 & 61,3 & 16,9 & 123,2 & 19,2 & 42,1 & 13,0 & 113,1 & 18,5 \\
1985 & 24,8 & 10,0 & 64,8 & 15,6 & 121,5 & 18,8 & 44,9 & 12,8 & 111,8 & 17,4 \\
1986 & 22,7 & 9,5 & 66,0 & 18,4 & 118,9 & 15,9 & 44,5 & 14,0 & 93,3 & 17,3 \\
1987 & 24,0 & 7,8 & 59,2 & 15,4 & 113,1 & 19,4 & 41,7 & 11,6 & 95,3 & 17,4 \\
1988 & 23,9 & 8,7 & 59,7 & 14,4 & 118,1 & 19,1 & 42,0 & 11,6 & 89,6 & 17,0 \\
1989 & 23,2 & 9,7 & 67,0 & 14,0 & 121,8 & 16,1 & 45,3 & 11,9 & 95,1 & 15,2 \\
1990 & 21,7 & 8,5 & 66,1 & 14,9 & 117,5 & 16,3 & 44,1 & 11,7 & 92,4 & 15,7 \\
1991 & 20,1 & 10,5 & 68,7 & 14,5 & 115,3 & 15,2 & 44,5 & 12,5 & 92,7 & 15,0 \\
1992 & 21,9 & 6,9 & 61,7 & 18,1 & 115,2 & 19,5 & 41,7 & 12,5 & 89,4 & 19,0 \\
1993 & 18,2 & 7,8 & 66,2 & 15,5 & 119,9 & 15,5 & 41,8 & 11,6 & 93,2 & 15,5 \\
1994 & 19,0 & 9,5 & 65,1 & 13,2 & 112,2 & 16,7 & 41,5 & 11,3 & 88,8 & 15,0 \\
\hline
\end{tabular}

Fuente/Source: OPS. Sistema de Información Técnica

*Tasa/Rate x 100.000 habitantes

M:Masculino/Male

F:Femenino/Female 
Cuadro 11.b - Tasa* de mortalidad de adolescentes y jóvenes por accidentes de vehículo a motor, según edad y sexo - Chile 1980-1994

Table 11.b - Mortality rate among adolescents and young adults due to traffic accidents, by age and sex-Chile 1980-1994

\begin{tabular}{|c|c|c|c|c|c|c|c|c|c|c|}
\hline \multirow{2}{*}{$\frac{\text { Edad }}{\text { Años }}$} & \multicolumn{2}{|c|}{10 a 14 años } & \multicolumn{2}{|c|}{15 a 19 años } & \multicolumn{2}{|c|}{20 a 24 años } & \multicolumn{2}{|c|}{10 a 19 años } & \multicolumn{2}{|c|}{15 a 24 años } \\
\hline & $M$ & $\mathrm{~F}$ & $M$ & $\mathrm{~F}$ & $M$ & $\mathrm{~F}$ & M & $\mathrm{F}$ & $M$ & $\mathrm{~F}$ \\
\hline 1980 & 5,9 & 4,0 & 12,0 & 2,7 & 25,1 & 3,5 & 9,0 & 3,3 & 18,1 & 3,0 \\
\hline 1981 & 6,4 & 4,5 & 9,5 & 2,5 & 23,6 & 4,6 & 8,0 & 3,5 & 16,1 & 3,5 \\
\hline 1984 & 2,5 & 1,0 & 6,7 & 2,1 & 12,0 & 1,9 & 4,6 & 1,5 & 9,5 & 2,1 \\
\hline 1985 & 2,9 & 1,3 & 4,7 & 1,3 & 12,5 & 2,7 & 3,8 & 1,3 & 8,7 & 2,0 \\
\hline 1986 & 3,0 & 1,5 & 7,9 & 1,8 & 9,2 & 2,5 & 5,5 & 1,6 & 8,6 & 2,2 \\
\hline 1987 & 3,7 & 2,3 & 6,8 & 2,4 & 12,8 & 3,3 & 5,3 & 2,4 & 9,8 & 2,9 \\
\hline 1988 & 4,0 & 1,5 & 6,0 & 2,1 & 11,9 & 2,9 & 5,0 & 1,8 & 9,1 & 2,5 \\
\hline 1989 & 3,7 & 1,0 & 6,2 & 2,4 & 14,4 & 1,8 & 5,0 & 1,7 & 10,4 & 2,1 \\
\hline 1990 & 2,9 & 1,5 & 8,0 & 1,6 & 13,2 & 2,6 & 5,5 & 1,6 & 10,6 & 2,1 \\
\hline 1991 & 4,7 & 1,8 & 10,4 & 2,6 & 17,0 & 3,1 & 7,6 & 2,2 & 13,8 & 2,9 \\
\hline 1992 & 7,7 & 3,1 & 6,4 & 1,8 & 22,5 & 4,9 & 9,0 & 4,3 & 14,7 & 3,4 \\
\hline 1993 & 5,5 & 2,9 & 14,0 & 3,7 & 22,8 & 3,4 & 9,7 & 3,3 & 18,4 & 3,6 \\
\hline 1994 & 5,1 & 3,2 & 12,6 & 4,2 & 22,8 & 4,4 & 8,8 & 3,7 & 17,7 & 4,3 \\
\hline
\end{tabular}

Fuente/Source:OPS. Sistema de Información Técnica

*Tasa/Rate x 100.000 habitantes

M:Masculino/Male

F:Femenino/Female

Cuadro 11.c - Tasa* de mortalidad de adolescentes y jóvenes por suicidio, según edad y sexo Chile 1980-1994

Table 11.c - Mortality rate among adolescents and young adults due to suicide, by age and sex-Chile 1980-1994

\begin{tabular}{lllllllllll}
\hline Edad & \multicolumn{1}{c}{10 a 14 años } & \multicolumn{2}{c}{15 a 19 años } & \multicolumn{2}{c}{20 a 24 años } & \multicolumn{2}{c}{10 a 19 años } & \multicolumn{2}{c}{15 a 24 años } \\
\hline Años & M & F & M & F & M & F & M & F & M & F \\
\hline 1980 & 0,5 & 0,0 & 7,2 & 2,9 & 14,5 & 2,7 & 3,8 & 1,4 & 10,6 & 2,8 \\
1981 & 1,1 & 0,2 & 8,1 & 3,6 & 12,0 & 3,5 & 4,6 & 1,9 & 9,9 & 3,6 \\
1984 & 1,3 & 0,5 & 8,0 & 3,2 & 14,2 & 4,1 & 4,6 & 1,9 & 11,1 & 3,7 \\
1985 & 1,4 & 0,2 & 7,2 & 2,7 & 13,7 & 1,6 & 4,3 & 1,5 & 10,6 & 2,2 \\
1986 & 1,1 & 0,7 & 5,4 & 3,5 & 11,6 & 2,4 & 3,3 & 2,1 & 8,6 & 3,0 \\
1987 & 1,0 & 0,2 & 5,7 & 3,1 & 12,5 & 3,0 & 3,3 & 1,6 & 9,1 & 3,0 \\
1988 & 0,8 & 1,0 & 5,6 & 2,8 & 12,1 & 3,3 & 3,2 & 1,9 & 8,9 & 3,1 \\
1989 & 1,6 & 0,3 & 7,5 & 1,6 & 14,7 & 2,6 & 4,6 & 1,0 & 11,2 & 2,1 \\
1990 & 1,5 & 0,5 & 6,2 & 2,5 & 15,1 & 1,3 & 3,9 & 1,5 & 10,7 & 1,9 \\
1991 & 0,8 & 1,2 & 7,4 & 3,1 & 15,3 & 2,4 & 4,1 & 2,2 & 11,4 & 2,8 \\
1992 & 1,0 & 0,5 & 5,7 & 2,2 & 9,4 & 2,6 & 3,3 & 1,3 & 7,6 & 2,4 \\
1993 & 1,4 & 0,3 & 4,4 & 2,0 & 14,6 & 2,3 & 2,9 & 1,2 & 9,5 & 2,1 \\
1994 & 0,3 & 0,6 & 6,2 & 1,3 & 15,6 & 1,6 & 3,2 & 1,0 & 10,9 & 1,5 \\
\hline
\end{tabular}

Fuente/Source:OPS. Sistema de Información Técnica

*Tasa/Rate x 100.000 habitantes

M:Masculino/Male

F: Femenino/Female

$43 \%$ entre las mujeres de igual edad, entre las fechas extremas del período estudiado. Las tasas se mantienen estables en los adolescentes de 10 y 14 años (Cuadro 11.b). Las tasas de mortalidad por accidentes de trafico son significativamente menores en comparación a las de los hombres, sin em- bargo presentan percentuales de crescimiento más elevados.

El suicidio es el segundo determinante de la mortalidad por causas externas en adolescentes y jóvenes en Chile; en los jóvenes de 15 a 24 años (aumento de 2,8\% en los varones y descenso de $46 \%$ en las mujeres), 
Cuadro 11.d - Tasa* de mortalidad de adolescentes y jóvenes por homicidio, según edad y sexo Chile 1980-1994

Table 11.d - Mortality rate among adolescents and young adults due to homicide, by age and sex Chile 1980-1994

\begin{tabular}{|c|c|c|c|c|c|c|c|c|c|c|}
\hline \multirow{2}{*}{$\begin{array}{l}\text { Edad } \\
\text { Años }\end{array}$} & \multicolumn{2}{|c|}{10 a 14 años } & \multicolumn{2}{|c|}{15 a 19 años } & \multicolumn{2}{|c|}{20 a 24 años } & \multicolumn{2}{|c|}{10 a 19 años } & \multicolumn{2}{|c|}{15 a 24 años } \\
\hline & M & $F$ & M & $\mathrm{F}$ & $M$ & $F$ & M & $\mathrm{F}$ & M & $\mathrm{F}$ \\
\hline 1980 & 0,6 & 0,2 & 2,3 & 0,3 & 6,1 & 1,1 & 1,5 & 0,2 & 7,7 & 0,7 \\
\hline 1981 & 0,2 & 0,0 & 3,1 & 0,8 & 8,9 & 1,6 & 1,6 & 0,4 & 5,8 & 1,2 \\
\hline 1984 & 0,8 & 0,2 & 3,9 & 0,3 & 7,5 & 1,0 & 2,4 & 0,2 & 5,8 & 0,7 \\
\hline 1985 & 0,0 & 0,7 & 3,1 & 0,2 & 9,3 & 1,1 & 1,6 & 0,4 & 6,3 & 0,6 \\
\hline 1986 & 0,6 & 0,2 & 3,2 & 0,8 & 9,9 & 0,6 & 1,9 & 0,5 & 7,4 & 0,7 \\
\hline 1987 & 1,0 & 0,2 & 5,7 & 3,1 & 12,5 & 3,0 & 3,3 & 1,6 & 9,1 & 3,0 \\
\hline 1988 & 0,6 & 0,5 & 3,5 & 0,5 & 9,7 & 1,4 & 2,1 & 0,5 & 6,7 & 1,0 \\
\hline 1989 & 1,6 & 0,3 & 7,5 & 1,6 & 14,7 & 2,6 & 4,6 & 1,0 & 11,2 & 2,1 \\
\hline 1990 & 1,5 & 0,5 & 6,2 & 2,5 & 15,1 & 1,3 & 3,9 & 1,5 & 10,7 & 1,9 \\
\hline 1991 & 0,8 & 1,2 & 7,4 & 3,1 & 15,3 & 2,4 & 4,1 & 2,2 & 11,4 & 2,8 \\
\hline 1992 & 0,5 & 0,0 & 3,1 & 0,3 & 8,5 & 0,8 & 1,8 & 0,2 & 5,8 & 0,6 \\
\hline 1993 & 0,3 & 0,0 & 3,9 & 0,5 & 10,3 & 0,8 & 2,1 & 0,2 & 7,1 & 0,7 \\
\hline 1994 & 0,5 & 0,2 & 4,9 & 0,2 & 6,9 & 1,6 & 2,6 & 0,2 & 5,9 & 0,9 \\
\hline
\end{tabular}

Fuente/Source:OPS. Sistema de Información Técnica

*Tasa/Rate x 100.000 habitantes

M:Masculino/Male

F:Femenino/Female

llegando a cifras de 10,9 por 100.000 en hombres de 15 a 24 años y de 1,5 por 100.000 en mujeres de igual edad (Cuadro 11.c).

Chile tiene tasas bajas de mortalidad por homicidio entre adolescentes y jóvenes, con una_disminuyción de $23 \%$ en la población de hombres de 15 a 24 años entre 1980 y 1994 (Cuadro 11.d). Cabe destacar que puede existir sesgo en esta información ya que Chile tiene alto porcentaje de muertes clasificadas como "lesiones en que se ignora si fueron accidental o intencionalmente infligidas", lo que sugiere cierta deficiencia en la calidad de certificación de la causa de muerte.

En suma, la disminución de la mortalidad por causas externas en adolescentes y jóvenes de Chile se explica por la disminución de los accidentes de tráfico. Las tasas de mortalidad registradas por suicidios y homicidios son bajas y hay una leve tendencia al descenso en la mortalidad por homicidios en los varones de 15 a 24 años.

\section{URUGUAY}

Las tasas de mortalidad por causas externas se mantienen estacionarias, con leves oscilaciones, en el período estudiado
(1980-1990) (Cuadros 1.3 a 1.5 en Anexo). En el grupo de jóvenes de 15 a 24 años la mortalidad por causas externas disminuye en un $20 \%$ en los varones y aumenta en un $29 \%$ en las mujeres. En los adolescentes de 10 a 14 años las tasas sufren oscilaciones a lo largo del período, sin cambios significativos en las fechas extremas (Cuadro 12.a).

La causa determinante de la mortalidad por causas externas en la población total de Uruguay son los accidentes de tráfico, los que han sufrido un descenso hasta el año 85 y posteriormente un ascenso, manteniendo tasas estables entre las fechas extremas. La tasa de mortalidad por suicidio es el segundo componente de la mortalidad por causas externas en la población total de Uruguay, con valores muy cercanos a las tasas de mortalidad por accidentes de tráfico. Hay una leve tendencia al aumento de los suicidios entre las fechas extremas. La tasa de mortalidad por homicidio en la población total de Uruguay es baja, pero se ha duplicado en el período estudiado (Cuadros 1.6, 1.9 y 1.12 en Anexo).

Los accidentes de tráfico tienen la mayor influencia en la mortalidad por causas externas en la población total adolescente 
Cuadro 12.a - Tasa* de mortalidad de adolescentes y jóvenes por causas externas, según edad y sexo - Uruguay 1980-1990

Table 12.a - Mortality rate among adolescents and young adults due to external causes, by age and sex-Uruguay 1980-1990

\begin{tabular}{lcrrrrrrrrr}
\hline Edad & \multicolumn{3}{c}{ 10 a 14 años } & \multicolumn{2}{c}{ 15 a 19 años } & \multicolumn{2}{c}{20 a 24 años } & \multicolumn{2}{c}{10 a 19 años } & \multicolumn{2}{c}{15 a 24 años } \\
\hline Años & M & \multicolumn{1}{c}{ F } & M & F & M & F & M & F & M & F \\
\hline 1980 & 24,4 & 9,3 & 79,8 & 21,3 & 110,0 & 15,2 & 52,2 & 15,4 & 94,0 & 18,4 \\
1981 & 21,0 & 10,1 & 62,6 & 19,0 & 100,0 & 26,3 & 41,7 & 14,6 & 80,3 & 22,6 \\
1982 & 26,2 & 5,0 & 73,6 & 13,4 & 93,8 & 23,5 & 49,4 & 9,2 & 83,3 & 18,4 \\
1983 & 16,3 & 4,1 & 58,0 & 14,5 & 72,8 & 13,8 & 36,3 & 9,2 & 65,2 & 14,2 \\
1984 & 15,9 & 11,9 & 48,3 & 12,9 & 80,0 & 12,9 & 31,2 & 12,4 & 60,6 & 12,6 \\
1985 & 31,9 & 10,0 & 46,6 & 12,9 & 76,5 & 13,8 & 38,7 & 11,4 & 57,9 & 12,9 \\
1986 & 24,1 & 10,7 & 50,0 & 17,9 & 80,9 & 19,0 & 36,2 & 14,1 & 61,7 & 17,8 \\
1987 & 24,3 & 13,4 & 55,3 & 20,2 & 87,7 & 22,8 & 38,8 & 16,6 & 70,9 & 21,5 \\
1988 & 20,6 & 12,5 & 67,5 & 19,7 & 95,6 & 22,1 & 42,7 & 15,9 & 77,2 & 20,2 \\
1989 & 26,8 & 8,8 & 83,8 & 31,2 & 108,8 & 21,2 & 54,0 & 19,5 & 95,5 & 26,5 \\
1990 & 26,1 & 7,3 & 64,7 & 26,8 & 87,7 & 20,4 & 44,7 & 16,7 & 75,3 & 23,8 \\
\hline
\end{tabular}

Fuente/Source:OPS. Sistema de Información Técnica

${ }^{*}$ Tasa/Rate $\times 100.000$ habitantes

M:Masculino/Male

F:Femenino/Female

Cuadro 12.b - Tasa* de mortalidad de adolescentes y jóvenes por accidentes de vehículo a motor, según edad y sexo - Uruguay 1980-1990

Table 12.b - Mortality rate among adolescents and young adults due to traffic accidents, by age and sex-Uruguay 1980-1990

\begin{tabular}{ccccccccccc}
\hline Edad & \multicolumn{1}{c}{ 10 a 14 años } & \multicolumn{2}{c}{ 15 a 19 años } & \multicolumn{2}{c}{20 a 24 años } & \multicolumn{2}{c}{10 a 19 años } & \multicolumn{2}{c}{15 a 24 años } \\
\hline Años & M & F & \multicolumn{1}{c}{ M } & F & M & F & M & F & M & F \\
\hline 1980 & 6,5 & 0,8 & 25,8 & 2,5 & 37,3 & 1,8 & 16,2 & 1,7 & 31,2 & 2,1 \\
1981 & 4,8 & 1,7 & 21,1 & 5,0 & 38,7 & 7,9 & 13,0 & 3,3 & 29,5 & 6,4 \\
1982 & 5,6 & 3,3 & 20,7 & 4,2 & 29,2 & 5,2 & 13,0 & 3,8 & 24,8 & 4,7 \\
1983 & 1,6 & 0,8 & 7,6 & 2,6 & 19,3 & 3,4 & 4,4 & 1,7 & 13,3 & 3,0 \\
1984 & 3,0 & 2,4 & 8,5 & 6,0 & 23,5 & 2,6 & 5,6 & 4,1 & 15,0 & 4,2 \\
1985 & 3,7 & 0,8 & 16,9 & 3,4 & 24,3 & 2,6 & 9,9 & 2,4 & 19,4 & 3,3 \\
1986 & 5,1 & 6,1 & 10,8 & 4,3 & 21,7 & 4,3 & 7,8 & 5,2 & 15,3 & 4,1 \\
1987 & 5,7 & 0,7 & 17,1 & 2,5 & 19,3 & 7,0 & 11,0 & 1,6 & 18,1 & 4,7 \\
1988 & 5,0 & 5,1 & 13,5 & 5,7 & 27,4 & 4,4 & 9,0 & 5,4 & 19,2 & 4,9 \\
1989 & 9,2 & 2,2 & 19,2 & 9,6 & 26,5 & 5,3 & 14,0 & 5,7 & 22,6 & 7,6 \\
1990 & 3,5 & 0,0 & 12,8 & 9,4 & 23,7 & 9,7 & 8,0 & 4,5 & 17,8 & 9,2 \\
\hline
\end{tabular}

Fuente/Source:OPS. Sistema de Información Técnica

*Tasa/Rate x 100.000 habitantes

M:Masculino/Male

F:Femenino/Female

y joven de Uruguay. En la población de hombres de 15 a 24 años las tasas han disminuido en un $43 \%$ entre 1980 y 1990, en cambio, en la población de mujeres de igual edad las cifras se han cuadruplicado. El cuociente hombres/mujeres de mortalidad por accidentes de tráfico en el grupo de 15 a 24 años es de 2/1 en 1990. Las tasas de mortalidad por accidentes de tráfico han disminuido tanto en niños como en niñas de 10 a 14 años (Cuadro 12.b).

La tasa de mortalidad por suicidio oscila durante el período estudiado y sufre una disminución de un $11 \%$ entre las fechas extremas en el grupo de hombres de 15 a 24 años, manteniéndose estable en las mujeres de igual edad. La mayor tasa de mortalidad por suicidio la presentan los hombres de 20 a 24 años. (19,3 por 100.000) (Cuadro 12.c).

La mortalidad registrada por homicidio 
Cuadro 12.c - Tasa* de mortalidad de adolescentes y jóvenes por suicidio, según edad y sexo Uruguay 1980-1990

Table 12.c - Mortality rate among adolescents and young adults due to suicide, by age and sex Uruguay 1980-1990

\begin{tabular}{ccccccccccc}
\hline Edad & \multicolumn{1}{c}{ 10 a 14 años } & \multicolumn{2}{c}{ 15 a 19 años } & \multicolumn{2}{c}{20 a 24 años } & \multicolumn{2}{c}{10 a 19 años } & \multicolumn{2}{c}{15 a 24 años } \\
\hline Años & M & F & M & F & M & F & M & F & M & F \\
\hline 1980 & 2,4 & 0,0 & 7,3 & 4,1 & 23,6 & 1,8 & 4,9 & 2,1 & 15,0 & 3,0 \\
1981 & 0,0 & 0,0 & 3,3 & 3,3 & 15,3 & 2,6 & 1,6 & 1,7 & 9,0 & 3,0 \\
1982 & 0,8 & 0,0 & 3,3 & 0,8 & 12,4 & 2,6 & 2,0 & 0,4 & 7,7 & 1,7 \\
1983 & 0,8 & 0,0 & 8,4 & 0,9 & 7,0 & 3,4 & 4,4 & 0,4 & 7,7 & 2,1 \\
1984 & 0,8 & 0,8 & 8,5 & 3,4 & 7,8 & 2,6 & 4,4 & 2,1 & 7,7 & 2,9 \\
1985 & 0,7 & 1,6 & 3,4 & 1,7 & 10,4 & 2,6 & 2,0 & 1,6 & 6,5 & 2,1 \\
1986 & 1,5 & 0,0 & 4,2 & 3,4 & 11,3 & 6,9 & 2,7 & 1,6 & 7,3 & 5,0 \\
1987 & 0,7 & 0,7 & 3,3 & 3,3 & 12,3 & 2,6 & 1,9 & 2,0 & 7,6 & 3,0 \\
1988 & 1,4 & 0,0 & 8,7 & 2,5 & 15,0 & 2,7 & 4,9 & 1,2 & 11,2 & 2,5 \\
1989 & 1,4 & 0,0 & 12,3 & 7,2 & 14,2 & 2,7 & 6,6 & 3,4 & 11,9 & 5,0 \\
1990 & 2,1 & 2,2 & 8,3 & 3,9 & 19,3 & 1,8 & 5,1 & 3,0 & 13,4 & 2,9 \\
\hline
\end{tabular}

Fuente/Source:OPS. Sistema de Información Técnica

*Tasa/Rate x 100.000 habitantes

M:Masculino/Male

F:Femenino/Female

Cuadro 12.d - Tasa* de mortalidad de adolescentes y jóvenes por homicidio, según edad y sexo Uruguay 1980-1990

Table 12.d - Mortality rate among adolescents and young adults due to homicide, by age and sex Uruguay 1980-1990

\begin{tabular}{llllllllllll}
\hline Edad & \multicolumn{2}{c}{10 a 14 años } & \multicolumn{2}{c}{15 a 19 años } & \multicolumn{2}{c}{ 20 a 24 años } & \multicolumn{2}{c}{10 a 19 años } & \multicolumn{2}{c}{15 a 24 años } \\
\hline Años & M & F & M & F & M & F & M & F & M & F \\
\hline 1980 & 0,8 & 0,0 & 3,2 & 1,6 & 3,6 & 3,6 & 2,0 & 0,8 & 3,4 & 2,6 \\
1981 & 0,8 & 0,0 & 3,3 & 1,7 & 6,3 & 3,5 & 2,0 & 0,8 & 4,7 & 2,6 \\
1982 & 0,0 & 0,0 & 5,0 & 0,8 & 2,7 & 1,7 & 2,4 & 0,4 & 3,8 & 1,3 \\
1983 & 0,0 & 0,8 & 0,0 & 1,7 & 7,0 & 4,3 & 0,0 & 1,3 & 3,4 & 3,0 \\
1984 & 0,0 & 0,8 & 1,7 & 1,7 & 5,2 & 0,9 & 0,8 & 1,2 & 3,3 & 1,3 \\
1985 & 1,5 & 0,0 & 0,8 & 0,9 & 4,3 & 2,6 & 1,2 & 0,4 & 2,4 & 1,7 \\
1986 & 0,0 & 0,8 & 2,5 & 2,6 & 3,5 & 1,7 & 1,2 & 1,6 & 2,8 & 2,1 \\
1987 & 1,1 & 0,0 & 2,9 & 3,3 & 5,3 & 6,1 & 1,9 & 1,5 & 8,0 & 4,7 \\
1988 & 1,4 & 0,7 & 2,4 & 0,8 & 3,5 & 5,3 & 1,9 & 0,8 & 2,8 & 2,9 \\
1989 & 2,1 & 0,0 & 4,6 & 1,6 & 13,3 & 1,8 & 3,3 & 0,8 & 8,6 & 1,7 \\
1990 & 0,7 & 0,7 & 3,8 & 3,1 & 7,9 & 3,5 & 2,2 & 1,9 & 5,7 & 3,3 \\
\hline
\end{tabular}

Fuente/Source:OPS. Sistema de Información Técnica

*Tasa/Rate x 100.000 habitantes

$\mathrm{M}$ : Masculino/Male

F:Femenino/Female

es baja en los adolescentes y jóvenes de Uruguay, pero aumenta en un $68 \%$ en los hombres de 15 a 24 años y en un $27 \%$ en las mujeres de igual edad, alcanzando tasas de 5,7 por 100.000 en hombres y 3,3 por $100.000 \mathrm{en}$ mujeres. El mayor aumento ocurre en el grupo de hombres de 20 a 24 años, alcanzando tasas de 7,9 por 100.000 en 1990 (12.d).

En resumen, la tasa de mortalidad por causas externas está determinada en primer lugar por los accidentes de tráfico en los adolescentes y jóvenes en Uruguay. Ambas tasas son decrecientes en hombres de 15 a 24 años y crecientes en mujeres de igual edad. El suicidio es el segundo determinante de la mortalidad por causas externas, con tendencia al descenso. Los homicidios son escasos y con leve tendencia al aumento. 
BRASIL

La tasa de mortalidad por causas externas en la población total de Brasil ha aumentado en un $25 \%$ en el período estudiado (1980-1995), a expensas de un aumento en la población masculina. En el grupo de varones adolescentes y jóvenes el aumento ha sido mas pronunciado (Cuadros 1.3 a 1.5 en Anexo), y el grupo mas afectado ha sido el de hombres de 15 a 19 años, con un ascenso de un $51 \%$ en las tasas de mortalidad por causas externas, llegando a valores de 122,2 por 100.000 en 1995. El grupo de jóvenes de 20 a 24 años es el que presenta mas altas tasas (195,3 por 100.000 en 1995) y ha incrementado la mortalidad por causas externas en un $47 \%$ en el período estudiado (Cuadro 13.a). Las adolescentes y jóvenes mujeres han mantenido tasas estables, cercanas a 24 por 100.000 , para el grupo de 15 a 24 años.

Los homicidios y los accidentes de tráfico son los componentes principales de la mortalidad por causas externas en la pobla- ción total. Los accidentes de tráfico han aumentado en un $24 \%$ en la población total entre 1980 y 1995 (Cuadro 1.6 en Anexo). También han aumentado en todos los grupos de adolescentes y jóvenes. El grupo de hombres de 15 a 19 años sufre un aumento de un $37 \%$ y un $22 \%$ las mujeres de igual edad. Los jóvenes de 20 a 24 años aumentan las tasas de mortalidad por accidentes de tráfico en un $33 \%$ y en un $28 \%$ las mujeres de igual edad (Cuadro 13.b).

La tasa de mortalidad por homicidios también ha aumentado en la población total en un porcentaje de $103 \%$ entre 1980 y 1995. A partir de 1990 la tasa de mortalidad por homicidios supera la tasa de mortalidad por accidentes de tráfico en la población total. La mortalidad por homicidios es el primer determinante de la mortalidad por causas externas en adolescentes y jóvenes (Cuadro 13.d) y ha experimentado un incremento significativo en todos los grupos de edad y sexo. Entre los adolescentes de 10 a 14 años las tasas se han triplicado entre 1980 y 1995 en ambos sexos. El grupo de adoles-

Cuadro 13.a - Tasa* de mortalidad de adolescentes y jóvenes por causas externas, según edad y sexo - Brasil 1980-1995

Table 13.a - Mortality rate among adolescents and young adults due to external causes, by age and sex-Brazil 1980-1995

\begin{tabular}{lcccccccccr}
\hline Edad & \multicolumn{2}{c}{10 a 14 años } & \multicolumn{2}{c}{15 a 19 años } & \multicolumn{2}{c}{20 a 24 años } & \multicolumn{2}{c}{10 a 19 años } & \multicolumn{2}{c}{15 a 24 años } \\
\hline Años & M & F & M & F & M & F & M & F & M & F \\
\hline 1980 & 30,1 & 12,7 & 81,2 & 22,7 & 141,5 & 23,2 & 55,4 & 17,6 & - & - \\
1981 & 29,8 & 12,5 & 83,3 & 23,1 & 142,5 & 24,0 & 56,4 & 17,7 & 110,9 & 23,5 \\
1982 & 28,4 & 12,3 & 82,1 & 21,4 & 140,7 & 23,8 & 54,9 & 16,8 & 109,9 & 22,6 \\
1983 & 28,7 & 12,5 & 87,9 & 21,8 & 146,0 & 23,3 & 57,8 & 17,1 & 116,0 & 22,5 \\
1984 & 28,3 & 11,4 & 95,3 & 21,5 & 156,8 & 23,1 & 60,9 & 16,3 & 124,9 & 22,3 \\
1985 & 31,2 & 12,4 & 101,6 & 22,6 & 158,9 & 23,5 & 65,2 & 17,3 & 129,9 & 23,0 \\
1986 & 32,7 & 13,4 & 112,8 & 24,9 & 177,2 & 26,4 & 71,2 & 18,9 & 144,6 & 25,6 \\
1987 & 30,5 & 12,0 & 109,8 & 21,6 & 175,2 & 23,7 & 68,5 & 16,6 & 142,1 & 22,6 \\
1988 & 30,5 & 12,2 & 109,8 & 21,3 & 174,5 & 23,6 & 68,4 & 16,5 & 141,4 & 22,4 \\
1989 & 32,2 & 12,1 & 126,4 & 22,5 & 198,6 & 25,5 & 77,1 & 17,1 & 161,5 & 24,0 \\
1990 & 28,2 & 11,2 & 122,7 & 21,0 & 201,2 & 25,2 & 72,1 & 15,8 & 159,4 & 23,0 \\
1991 & 26,4 & 10,8 & 117,6 & 21,5 & 196,5 & 25,0 & 68,8 & 15,8 & 154,5 & 23,1 \\
1992 & 26,4 & 9,8 & 109,2 & 19,2 & 183,8 & 22,3 & 64,9 & 14,3 & 144,1 & 20,7 \\
1993 & 27,2 & 11,4 & 114,9 & 21,0 & 187,6 & 23,9 & 74,0 & 15,9 & 148,9 & 22,4 \\
1994 & 28,3 & 12,9 & 122,9 & 24,1 & 197,9 & 25,0 & 72,3 & 18,2 & 158,0 & 24,5 \\
1995 & 28,5 & 13,3 & 122,2 & 23,4 & 195,3 & 25,1 & 74,0 & 18,3 & 156,4 & 24,2 \\
\hline
\end{tabular}

Fuente/Source:OPS.Sistema de Información Técnica

*Tasa/Rate x 100.000 habitantes

M:Masculino/Male

F: Femenino/Female 
Cuadro 13.b - Tasa* de mortalidad de adolescentes y jóvenes por accidentes de vehículo a motor, según edad y sexo - Brasil 1980-1995

Table 13.b - Mortality rate among adolescents and young adults due to traffic accidents, by age and sex-Brazil 1980-1995

\begin{tabular}{ccccccccccrr}
\hline Edad & \multicolumn{1}{c}{10 a 14 años } & \multicolumn{2}{c}{15 a 19 años } & \multicolumn{2}{c}{20 a 24 años } & \multicolumn{2}{c}{10 a 19 años } & \multicolumn{2}{c}{15 a 24 años } \\
\hline Años & M & F & M & F & M & F & M & $F$ & $M$ & F \\
\hline 1980 & 9,2 & 4,9 & 18,6 & 7,4 & 33,7 & 7,2 & 13,9 & 6,1 & - & - \\
1981 & 9,1 & 4,6 & 19,5 & 6,5 & 32,0 & 6,6 & 14,2 & 5,5 & 25,3 & 65,0 \\
1982 & 9,8 & 4,3 & 21,3 & 7,4 & 33,3 & 7,7 & 15,5 & 5,9 & 27,0 & 7,6 \\
1983 & 8,7 & 4,3 & 19,4 & 6,5 & 32,2 & 6,7 & 14,0 & 5,4 & 25,6 & 6,6 \\
1984 & 8,9 & 4,0 & 21,7 & 7,2 & 34,4 & 7,1 & 15,2 & 5,6 & 34,5 & 7,1 \\
1985 & 10,6 & 5,0 & 23,0 & 8,2 & 36,7 & 8,2 & 16,6 & 6,5 & 29,8 & 8,2 \\
1986 & 11,7 & 5,3 & 28,3 & 9,3 & 45,7 & 10,1 & 19,7 & 7,2 & 36,9 & 9,7 \\
1987 & 10,3 & 4,7 & 24,9 & 8,3 & 41,7 & 8,6 & 17,3 & 6,4 & 33,2 & 8,5 \\
1988 & 10,8 & 5,2 & 23,9 & 7,9 & 41,6 & 8,6 & 17,0 & 6,5 & 32,5 & 8,2 \\
1989 & 11,5 & 5,0 & 25,8 & 8,0 & 43,5 & 9,2 & 18,3 & 6,4 & 34,4 & 8,6 \\
1990 & 9,9 & 4,8 & 24,9 & 7,9 & 43,2 & 8,9 & 16,9 & 6,3 & 33,4 & 8,4 \\
1991 & 9,2 & 4,9 & 24,6 & 8,1 & 43,6 & 8,8 & 16,4 & 6,4 & 33,5 & 8,4 \\
1992 & 9,3 & 4,0 & 22,9 & 7,3 & 39,0 & 8,1 & 15,6 & 5,5 & 30,4 & 7,7 \\
1993 & 9,0 & 5,0 & 23,4 & 8,1 & 39,4 & 8,4 & 15,6 & 6,4 & 30,8 & 8,2 \\
1994 & 10,1 & 5,0 & 25,5 & 8,9 & 43,2 & 9,4 & 17,2 & 6,8 & 33,8 & 9,1 \\
1995 & 10,2 & 5,7 & 25,6 & 9,0 & 44,9 & 9,2 & 17,7 & 7,3 & 34,6 & 9,1 \\
\hline
\end{tabular}

Fuente/Source:OPS. Sistema de Información Técnica

*Tasa/Rate $\times 100.000$ habitantes

M:Masculino/Male

F:Femenino/Female

Cuadro 13.c - Tasa* de mortalidad de adolescentes y jóvenes por suicidio, según edad y sexo Brasil 1980-1995

Table 13.c - Mortality rate among adolescents and young adults due to suicide, by age and sex Brazil 1980-1995

\begin{tabular}{ccccccccccr}
\hline Edad & \multicolumn{2}{c}{10 a 14 años } & \multicolumn{2}{c}{15 a 19 años } & \multicolumn{2}{c}{20 a 24 años } & \multicolumn{2}{c}{10 a 19 años } & \multicolumn{2}{c}{15 a 24 años } \\
\hline Años & M & F & M & F & M & F & M & F & M & F \\
\hline 1980 & 0,4 & 0,5 & 3,0 & 3,1 & 6,6 & 3,3 & 1,7 & 1,8 & - & - \\
1981 & 0,5 & 0,5 & 3,7 & 3,4 & 7,8 & 3,3 & 2,1 & 2,0 & 5,6 & 3,4 \\
1982 & 0,4 & 0,6 & 3,1 & 2,6 & 7,2 & 3,1 & 1,7 & 1,6 & 5,1 & 2,8 \\
1983 & 0,5 & 0,6 & 3,5 & 3,1 & 7,3 & 3,4 & 2,0 & 1,8 & 5,3 & 3,2 \\
1984 & 0,4 & 0,6 & 2,8 & 2,5 & 7,2 & 2,6 & 1,5 & 1,5 & 5,0 & 2,5 \\
1985 & 0,4 & 0,6 & 2,8 & 1,9 & 6,8 & 2,2 & 1,6 & 1,2 & 4,8 & 2,0 \\
1986 & 0,4 & 0,5 & 3,1 & 2,4 & 6,7 & 2,3 & 1,7 & 1,4 & 4,9 & 2,4 \\
1987 & 0,4 & 0,4 & 3,4 & 2,0 & 6,8 & 2,2 & 1,9 & 1,2 & 5,1 & 2,1 \\
1988 & 0,4 & 0,3 & 2,8 & 1,8 & 6,6 & 2,2 & 1,6 & 1,0 & 4,7 & 2,0 \\
1989 & 0,3 & 0,4 & 3,3 & 1,9 & 7,0 & 1,7 & 1,7 & 1,2 & 5,1 & 1,8 \\
1990 & 0,4 & 0,4 & 3,4 & 1,7 & 7,5 & 2,6 & 1,8 & 1,0 & 5,3 & 2,1 \\
1991 & 0,5 & 0,4 & 3,5 & 2,0 & 7,8 & 2,3 & 1,9 & 1,1 & 5,5 & 2,1 \\
1992 & 0,4 & 0,4 & 3,7 & 1,7 & 8,1 & 2,2 & 1,9 & 1,0 & 5,7 & 1,9 \\
1993 & 0,6 & 0,3 & 4,2 & 2,1 & 8,8 & 2,5 & 2,3 & 1,2 & 6,3 & 2,3 \\
1994 & 0,5 & 0,5 & 4,7 & 2,3 & 9,1 & 2,7 & 2,5 & 1,4 & 6,7 & 2,5 \\
1995 & 0,4 & 0,4 & 4,5 & 2,2 & 9,6 & 2,3 & 2,4 & 1,3 & 6,9 & 2,2 \\
\hline
\end{tabular}

Fuente/Source:OPS. Sistema de Información Técnica

*Tasa/Rate $\mathrm{x} 100.000$ habitantes

M:Masculino/Male

F:Femenino/Female 
Cuadro 13.d - Tasa* de mortalidad de adolescentes y jóvenes por homicidio, según edad y sexo Brasil 1980-1995

Table 13.d - Mortality rate among adolescents and young adults due to homicide, by age and sex Brazil 1980-1995

\begin{tabular}{ccccccccccr}
\hline Edad & \multicolumn{1}{c}{ 10 a 14 años } & \multicolumn{2}{c}{ 15 a 19 años } & \multicolumn{2}{c}{20 a 24 años } & \multicolumn{2}{c}{10 a 19 años } & \multicolumn{2}{c}{15 a 24 años } \\
\hline Años & M & F & M & F & M & F & M & F & M & F \\
\hline 1980 & 1,3 & 0,5 & 19,3 & 2,9 & 43,6 & 3,6 & 10,2 & 1,7 & - & - \\
1981 & 1,5 & 0,6 & 20,3 & 2,6 & 45,8 & 4,6 & 10,8 & 1,6 & 32,2 & 3,5 \\
1982 & 1,6 & 0,6 & 19,9 & 2,5 & 44,2 & 4,1 & 10,6 & 1,5 & 31,5 & 3,3 \\
1983 & 1,7 & 0,8 & 23,7 & 3,1 & 46,3 & 4,4 & 12,5 & 1,9 & 34,7 & 3,8 \\
1984 & 1,8 & 0,6 & 28,2 & 3,5 & 54,2 & 4,4 & 14,6 & 2,0 & 41,0 & 3,9 \\
1985 & 2,2 & 0,7 & 32,5 & 3,5 & 53,0 & 5,0 & 16,9 & 2,1 & 42,6 & 4,2 \\
1986 & 2,5 & 1,0 & 34,6 & 3,5 & 56,0 & 4,5 & 17,9 & 2,2 & 45,2 & 4,0 \\
1987 & 2,6 & 0,8 & 37,7 & 3,5 & 63,8 & 4,7 & 19,4 & 2,1 & 50,5 & 4,1 \\
1988 & 2,7 & 0,7 & 37,2 & 3,5 & 62,7 & 4,8 & 19,2 & 2,1 & 49,7 & 4,1 \\
1989 & 3,1 & 1,2 & 48,8 & 4,4 & 82,5 & 6,4 & 24,9 & 2,7 & 65,2 & 5,3 \\
1990 & 3,4 & 1,1 & 55,2 & 4,3 & 95,3 & 6,3 & 27,4 & 2,6 & 73,9 & 5,3 \\
1991 & 3,1 & 1,2 & 49,7 & 4,7 & 85,6 & 6,1 & 24,8 & 2,8 & 66,5 & 5,3 \\
1992 & 2,9 & 1,0 & 43,5 & 3,7 & 78,5 & 5,3 & 21,8 & 2,2 & 59,8 & 4,4 \\
1993 & 3,3 & 1,0 & 49,6 & 4,0 & 83,7 & 5,8 & 24,8 & 2,4 & 65,6 & 4,9 \\
1994 & 2,8 & 1,3 & 52,9 & 5,4 & 93,3 & 5,5 & 26,1 & 3,2 & 71,8 & 5,4 \\
1995 & 3,9 & 1,5 & 56,4 & 5,4 & 94,4 & 6,8 & 29,4 & 3,4 & 74,2 & 6,0 \\
\hline
\end{tabular}

Fuente/Source:OPS. Sistema de Información Técnica

*Tasa/Rate x 100.000 habitantes

M:Masculino/Male

F: Femenino/Female

centes hombres de 15 a 19 años ha incrementado las tasas en un $192 \%$, llegando a valores de 56,4 por 100.000 en 1995. Las mujeres de igual edad incrementan sus tasas en un $86 \%$, alcanzando valores de 5,4 por 100.000. El grupo de hombres de 20 a 24 años es el que presenta mayores tasas de mortalidad por homicidio, con cifras de 94,4 por 100.000 en 1995, con un incremento de un $117 \%$ desde 1980 . Hay una diferencia marcada de sexo en esta edad, con un cuociente hombres/mujeres de 14/1 en 1995.

La mortalidad por suicidio se ha mantenido estable en la población total durante este período (Cuadro 1.9 en Anexo), en cambio, en la población de 15 a 24 años se observa un incremento en el lapso estudiado (Cuadro 13.c). Los varones de 20 a 24 años aumentan las tasas en un $45 \%$ entre 1980 y 1995 y el incremento es mas pronunciado a partir de 1989.

En resumen, en Brasil el aumento de la mortalidad por causas externas en los adolescentes se debe principalmente a un au- mento de los accidentes de tráfico y los homicidios, y secundariamente al aumento de los suicidios. Los adolescentes y jóvenes aumentan en forma notoria la mortalidad por homicidios durante este período, especialmente el grupo de 15 a 19 años.

\section{E. Países del Caribe}

\section{CUBA}

La mortalidad por causas externas sufre un aumento progresivo de un $28 \%$ en la población total de Cuba entre las fechas extremas estudiadas:1980-1996. (23\% de aumento en hombres y $27 \%$ en mujeres) (Cuadros 1.3 a 1.5 en Anexo).

En la población adolescente y joven la mortalidad por causas externas disminuye en el mismo período para las mujeres a toda edad: $56 \%$ de descenso entre las mujeres de 10 a 14 años, 55\% en mujeres de 15 a 19 años y $54 \%$ entre mujeres de 20 a 24 años). En el grupo de hombres de 15 a 19 años la tasa 
Cuadro 14.a - Tasa* de mortalidad de adolescentes y jóvenes por causas externas, según edad y sexo - Cuba 1980-1996

Table 14.a - Mortality rate among adolescents and young adults due to external causes, by age and sex-Cuba 1980-1996

\begin{tabular}{ccccccccccc}
\hline Edad & \multicolumn{2}{c}{10 a 14 años } & \multicolumn{2}{c}{15 a 19 años } & \multicolumn{2}{c}{20 a 24 años } & \multicolumn{2}{c}{10 a 19 años } & \multicolumn{2}{c}{15 a 24 años } \\
\hline Años & M & F & M & F & M & F & M & F & M & F \\
\hline 1980 & 27,1 & 22,5 & 65,6 & 66,4 & 106,8 & 70,4 & 45,7 & 44,1 & 81,9 & 68,0 \\
1981 & 27,3 & 21,0 & 62,4 & 66,2 & 98,2 & 70,1 & 44,5 & 43,5 & 76,8 & 67,8 \\
1982 & 30,1 & 20,3 & 67,1 & 63,8 & 94,5 & 70,6 & 48,5 & 42,1 & 78,6 & 66,7 \\
1983 & 22,0 & 14,1 & 61,7 & 54,1 & 85,9 & 57,8 & 41,9 & 34,2 & 72,4 & 55,8 \\
1984 & 27,0 & 17,0 & 64,5 & 53,9 & 98,5 & 58,6 & 45,9 & 35,6 & 79,7 & 57,0 \\
1985 & 27,8 & 17,6 & 61,9 & 54,2 & 99,5 & 61,6 & 45,3 & 36,3 & 80,1 & 59,1 \\
1986 & 29,4 & 17,2 & 67,6 & 58,8 & 105,0 & 60,4 & 49,5 & 39,1 & 86,9 & 61,3 \\
1987 & 35,1 & 15,4 & 68,4 & 54,3 & 112,4 & 62,0 & 53,2 & 36,5 & 90,2 & 58,2 \\
1988 & 39,0 & 16,3 & 79,6 & 54,1 & 108,1 & 54,5 & 61,8 & 37,6 & 96,3 & 56,3 \\
1989 & 30,5 & 16,2 & 75,9 & 49,1 & 110,0 & 52,8 & 56,7 & 35,3 & 93,2 & 51,1 \\
1990 & 29,6 & 13,9 & 73,9 & 37,3 & 121,1 & 51,0 & 55,6 & 27,6 & 97,4 & 44,3 \\
1991 & 31,3 & 15,1 & 80,7 & 49,5 & 119,0 & 53,5 & 60,1 & 35,2 & 100,7 & 51,6 \\
1992 & 27,9 & 13,4 & 74,2 & 43,6 & 104,1 & 46,1 & 54,3 & 30,6 & 90,8 & 45,2 \\
1993 & 27,9 & 15,0 & 75,4 & 36,4 & 102,9 & 40,3 & 54,0 & 26,7 & 91,8 & 39,1 \\
1994 & 28,3 & 12,4 & 73,3 & 31,7 & 103,7 & 42,6 & 51,9 & 22,5 & 90,8 & 38,0 \\
1995 & 24,1 & 13,0 & 80,1 & 32,5 & 99,8 & 41,2 & 52,0 & 22,7 & 91,6 & 37,6 \\
1996 & 20,9 & 9,8 & 67,0 & 30,1 & 91,0 & 32,6 & 43,1 & 19,6 & 81,0 & 31,6 \\
\hline
\end{tabular}

Fuente/Source: OPS. Sistema de Información Técnica

*Tasa/Rate x 100.000 habitantes

M:Masculino/Male

F:Femenino/Female

Cuadro 14.b - Tasa* de mortalidad de adolescentes y jóvenes por accidentes de vehículo a motor, según edad y sexo - Cuba 1992-1996

Table 14.b - Mortality rate among adolescents and young adults due to traffic accidents, by age and sex-Cuba 1992-1996

\begin{tabular}{ccccccccccc}
\hline Edad & \multicolumn{2}{c}{ 10 a 14 años } & \multicolumn{2}{c}{ 15 a 19 años } & \multicolumn{2}{c}{20 a 24 años } & \multicolumn{2}{c}{10 a 19 años } & \multicolumn{2}{c}{15 a 24 años } \\
\hline Años & M & F & M & \multicolumn{1}{c}{ F } & M & F & M & F & M & F \\
\hline 1992 & 10,1 & 3,1 & 28,0 & 11,1 & 32,6 & 9,8 & 20,3 & 7,7 & 30,6 & 10,4 \\
1993 & 12,2 & 7,5 & 27,0 & 7,3 & 28,6 & 7,7 & 20,3 & 7,4 & 28,2 & 7,6 \\
1994 & 11,3 & 5,5 & 26,5 & 11,5 & 29,3 & 8,7 & 19,3 & 8,6 & 18,0 & 9,9 \\
1995 & 8,1 & 5,3 & 24,0 & 8,5 & 31,5 & 9,6 & 16,0 & 6,9 & 28,4 & 9,2 \\
1996 & 8,6 & 5,2 & 23,7 & 8,9 & 30,0 & 7,6 & 15,9 & 7,0 & 27,4 & 8,1 \\
\hline
\end{tabular}

Fuente/Source:OPS. Sistema de Información Técnica

*Tasa/Rate x 100.000 habitantes

M: Masculino/Male

F:Femenino/Female

aumenta en un $2 \%$ en el mismo período y en los hombres de 20 a 24 años disminuye en un 15\% (Cuadro 14.a).

Sólo se dispone en Cuba de información por subgrupo de causas externas para los años 1992 a 1996, lo que limita el análisis de tendencias de mortalidad.

El componente principal de la mortalidad por causas externas en la población to- tal de Cuba son los suicidios, con tasas de 18,3 por 100.000 en 1996, seguido por los accidentes de tráfico, con tasas de 16,9 por 100.000 en 1996. Los accidentes de tráfico solo predominan en la población total de varones, con tasas de 26,6 por 100.000 en 1996 (Cuadros 1.9, 1.6 y 1.7 en Anexo) .

En la población de hombres de 15 a 24 años, homicidios, suicidios y accidentes de 
Cuadro 14.c - Tasa* de mortalidad de adolescentes y jóvenes por suicidio, según edad y sexo Cuba 1992-1996

Table 14.c - Mortality rate among adolescents and young adults due to suicide, by age and sex -Cuba 1992-1996

\begin{tabular}{llcrrrrrrrrr}
\hline Edad & \multicolumn{2}{c}{ 10 a 14 años } & \multicolumn{2}{c}{ 15 a 19 años } & \multicolumn{2}{c}{ 20 a 24 años } & \multicolumn{2}{c}{ 10 a 19 años } & \multicolumn{2}{c}{15 a 24 años } \\
\hline Años & M & F & M & F & M & F & M & F & M & F \\
\hline 1992 & 1,3 & 4,5 & 8,5 & 21,6 & 20,7 & 22,7 & 5,4 & 14,2 & 15,2 & 22,3 \\
1993 & 0,5 & 1,9 & 8,8 & 19,0 & 19,2 & 16,9 & 5,0 & 11,3 & 14,8 & 18,1 \\
1994 & 0,3 & 1,1 & 6,9 & 12,7 & 19,7 & 18,3 & 3,8 & 7,2 & 14,3 & 16,0 \\
1995 & 0,3 & 2,7 & 10,5 & 15,5 & 17,5 & 19,5 & 5,3 & 9,0 & 14,6 & 17,9 \\
1996 & 0,5 & 1,8 & 6,1 & 12,5 & 20,1 & 13,5 & 3,2 & 7,0 & 14,2 & 13,1 \\
\hline
\end{tabular}

Fuente/Source: OPS. Sistema de Información Técnica

*Tasa/Rate x 100.000 habitantes

M:Masculino/Male

F:Femenino/Female

Cuadro 14.d - Tasa* de mortalidad de adolescentes y jóvenes por homicidio, según edad y sexo Cuba 1992-1996

Table 14.d - Mortality rate among adolescents and young adults due to homicide, by age and sex Cuba 1992-1996

\begin{tabular}{ccccccccccc}
\hline Edad & \multicolumn{2}{c}{10 a 14 años } & \multicolumn{2}{c}{ 15 a 19 años } & \multicolumn{2}{c}{20 a 24 años } & \multicolumn{2}{c}{10 a 19 años } & \multicolumn{2}{c}{15 a 24 años } \\
\hline Años & M & F & M & F & M & F & M & F & M & F \\
\hline 1992 & 2,1 & 0,6 & 9,3 & 5,5 & 17,5 & 7,6 & 6,2 & 3,3 & 13,8 & 6,7 \\
1993 & 2,4 & 1,1 & 11,0 & 3,0 & 24,0 & 8,2 & 7,1 & 2,1 & 18,5 & 6,0 \\
1994 & 1,3 & 0,3 & 11,2 & 3,7 & 23,7 & 8,5 & 6,5 & 2,1 & 18,4 & 6,5 \\
1995 & 1,0 & 0,8 & 14,8 & 4,0 & 22,8 & 6,2 & 7,9 & 2,4 & 19,5 & 5,3 \\
1996 & 0,5 & 0,5 & 10,9 & 3,9 & 19,9 & 6,6 & 5,5 & 2,1 & 16,1 & 5,5 \\
\hline
\end{tabular}

Fuente/Source:OPS. Sistema de Información Técnica

*Tasa/Rate x 100.000 habitantes

M:Masculino/Male

F:Femenino/Female

tráfico comparten tasas de mortalidad semejantes en 1994 (accidentes de tráfico:18 por 100.000; homicidios: 18,4 por $100.000 \mathrm{y}$ suicidios: 14,3 por 100.000 ). Ya en 1996, la tasa por accidente de vehículo a motor entre los hombres de 15 a 24 años aumentó para 27,4 por 100.000 , superando las tasas por homicidios $(16,1)$ y por suicidio $(14,2)$ en el mismo año. En cambio, en la población de mujeres de 15 a 24 años la tasa de mortalidad por suicidio es predominante y alcanza tasas de 13,1 por 100.000 en 1996, en relación a la tasa de mortalidad por accidentes de tráfico de 8,1 por 100.000 y por homicidios de 5,5 por 100.000. En el grupo de adolescentes de 10 a 14 años predomina la mortalidad por accidentes de tráfico, en ambos sexos (Cuadros 14.b a 14.d).

En suma, la mortalidad por causas externas ha aumentado en la población total en
Cuba entre 1980 y 1996 y también ha aumentado levemente en el grupo de adolescentes varones de 15 a 19 años. El suicidio adquiere gran importancia como determinante de causa externa de mortalidad en los hombres de 20 a 24 años entre 1992 y 1996 y, entre las mujeres del mismo grupo etário disminuyen significativamente en el mismo período.

\section{PUERTO RICO}

La mortalidad por causas externas en la población total de Puerto Rico es levemente descendente entre 1980 y 1983, elevándose posteriormente en un $33 \%$ entre los 1983 y 1992, hasta llegar a tasas de 72,1 por 100.000 el último ano estudiado (Cuadros 1.3 a 1.5 en Anexo). La misma tendencia a un descenso inicial de 23\% hasta el año 1983 y un segundo período de ascenso de $67 \%$ 
en las tasas de mortalidad por causas externas se observa en los grupos de hombres de 15 a 24 años, con un ascenso total de $28 \%$ entre 1980 y 1992. La mortalidad del grupo de mujeres de 15 a 24 años se ha mantenido estacionaria, con leves oscilaciones, llegando a 19,4 por 100.000 en 1992. Llama la atención las tasas persistentemente altas de mortalidad por causas externas en el grupo de adolescentes de 10 a 14 años en el período estudiado, alcanzando valores de 18,9 por 100.000 en el grupo de hombres y 4,9 por 100.000 en las mujeres (Cuadro 15.a).

Las causas determinantes de la tasa de mortalidad por causas externas de la población total de Puerto Rico son los accidentes de tráfico y los homicidios, con predominio de estos últimos desde el año 1990. Las tasas de mortalidad por homicidios en la población total aumenta en un $57 \%$ en el período estudiado, con una disminución inicial hasta 1984 y posterior ascenso hasta 1992 (Cuadros 1.6 y 1.12 en Anexo).

En los adolescentes y jóvenes el homicidio es el determinante principal de la mortalidad por causas externas. La misma tendencia de descenso inicial hasta el año 1984 y ascenso progresivo posterior se observa en las tasas de mortalidad por homicidio en los grupos de hombres y mujeres de 15 a 24 años. La población de hombres de 15 a 19 años aumenta las tasas en un $238 \%$ entre 1983 y 1992, llegando a valores de 65,3 por 100.000 en 1992, y en un $127 \%$ en el de 20 a 24 años, con tasas de 125,7 por 100.000 en 1992. Las tasas en los hombres son 10 veces mayores o mas, en relación a las mujeres a partir del año 1984. Las tasas de mortalidad por homicidios en los adolescentes de $10 \mathrm{a}$ 14 años son bajas, con oscilaciones en el período, sin tendencia fija por el escaso número de casos (Cuadro 15.d).

Las tasas de accidentes de tráfico y suicidio en la población total y en adolescentes y jóvenes se mantiene estable, con oscilaciones leves en el período estudiado Cuadros 1.6 a 1.11 en Anexo, 15.b y 15.c).

En resumen, las tasas de mortalidad por homicidio son determinantes del aumento de la mortalidad por causa externa en los adolescentes y jóvenes de Puerto Rico. El aumento mayor en las tasas se observa entre los años 1983 y 1992, en el grupo de hombres de 15 a 19 años. Las tasas mas altas de mortalidad por homicidio las presenta el grupo de hombres de 20 a 24 años.

Cuadro 15.a - Tasa* de mortalidad de adolescentes y jóvenes por causas externas, según edad y sexo - Puerto Rico 1980-1992

Table 15.a - Mortality rate among adolescents and young adults due to external causes, by age and sex-Puerto Rico 1980-1992

\begin{tabular}{lcrrrrrrrrr}
\hline Edad & \multicolumn{2}{c}{10 a 14 años } & \multicolumn{2}{c}{15 a 19 años } & \multicolumn{2}{c}{20 a 24 años } & \multicolumn{2}{c}{10 a 19 años } & \multicolumn{2}{c}{15 a 24 años } \\
\hline Años & M & F & M & F & M & F & M & F & M & F \\
\hline 1980 & 22,0 & 3,6 & 101,8 & 17,8 & 157,7 & 22,2 & 61,4 & 10,7 & 126,1 & 19,8 \\
1981 & 18,0 & 9,7 & 95,2 & 13,8 & 152,7 & 15,9 & 56,0 & 11,7 & 120,5 & 14,7 \\
1982 & 22,2 & 6,1 & 89,6 & 11,0 & 142,6 & 20,3 & 55,2 & 8,5 & 113,7 & 15,4 \\
1983 & 18,9 & 8,6 & 61,5 & 13,0 & 137,6 & 23,8 & 39,7 & 10,8 & 97,0 & 18,3 \\
1984 & 17,9 & 6,8 & 83,5 & 20,9 & 117,1 & 20,8 & 49,7 & 13,8 & 99,7 & 20,8 \\
1985 & 27,5 & 10,5 & 89,8 & 19,9 & 132,9 & 16,7 & 57,7 & 15,1 & 110,8 & 18,3 \\
1986 & 23,2 & 7,4 & 93,6 & 17,9 & 144,3 & 24,5 & 57,2 & 12,5 & 118,3 & 21,2 \\
1987 & 24,1 & 3,7 & 80,5 & 17,8 & 146,3 & 22,2 & 51,4 & 10,6 & 112,1 & 20,0 \\
1988 & 20,3 & 4,8 & 88,8 & 14,6 & 149,3 & 22,7 & 53,5 & 9,6 & 117,4 & 18,5 \\
1989 & 17,3 & 4,8 & 83,5 & 20,0 & 142,6 & 25,2 & 49,6 & 12,2 & 110,8 & 22,5 \\
1990 & 18,5 & 3,6 & 100,6 & 9,3 & 161,7 & 22,4 & 58,7 & 6,4 & 128,7 & 15,6 \\
1991 & 20,9 & 4,8 & 141,1 & 16,6 & 189,6 & 20,9 & 80,3 & 10,6 & 163,5 & 18,6 \\
1992 & 18,9 & 4,9 & 117,6 & 12,8 & 212,8 & 26,7 & 68,4 & 8,9 & 161,9 & 19,4 \\
\hline
\end{tabular}

Fuente/Source:OPS. Sistema de Información Técnica

*Tasa/Rate x 100.000 habitantes

M:Masculino/Male

F:Femenino/Female 
Cuadro 15.b - Tasa* de mortalidad de adolescentes y jóvenes por accidentes de vehículo a motor, según edad y sexo - Puerto Rico 1980-1992

Table 15.b - Mortality rate among adolescents and young adults due to traffic accidents, by age and sex - Puerto Rico 1980-1992

\begin{tabular}{|c|c|c|c|c|c|c|c|c|c|c|}
\hline \multirow{2}{*}{$\frac{\text { Edad }}{\text { Años }}$} & \multicolumn{2}{|c|}{10 a 14 años } & \multicolumn{2}{|c|}{15 a 19 años } & \multicolumn{2}{|c|}{20 a 24 años } & \multicolumn{2}{|c|}{10 a 19 años } & \multicolumn{2}{|c|}{15 a 24 años } \\
\hline & M & $\mathrm{F}$ & M & $\mathrm{F}$ & M & $\mathrm{F}$ & M & $\mathrm{F}$ & M & $\mathrm{F}$ \\
\hline 1980 & 5,8 & 1,2 & 29,0 & 8,9 & 53,1 & 8,3 & 17,3 & 5,1 & 39,5 & 8,6 \\
\hline 1981 & 5,8 & 3,6 & 29,3 & 5,4 & 44,3 & 7,6 & 17,4 & 4,5 & 35,9 & 6,4 \\
\hline 1982 & 7,6 & 2,4 & 31,1 & 5,5 & 36,0 & 8,1 & 19,1 & 4,0 & 33,3 & 6,7 \\
\hline 1983 & 5,3 & 4,9 & 20,5 & 5,0 & 41,1 & 11,9 & 12,7 & 4,9 & 30,1 & 8,3 \\
\hline 1984 & 3,8 & 3,1 & 24,7 & 9,5 & 37,7 & 7,1 & 16,3 & 6,3 & 30,9 & 8,3 \\
\hline 1985 & 13,2 & 4,3 & 28,7 & 8,3 & 40,3 & 7,1 & 20,7 & 6,3 & 34,3 & 7,7 \\
\hline 1986 & 8,9 & 5,5 & 26,1 & 8,3 & 43,0 & 11,0 & 17,2 & 6,9 & 34,3 & 9,6 \\
\hline 1987 & 7,1 & 1,8 & 30,8 & 10,2 & 42,2 & 11,1 & 18,5 & 5,9 & 36,3 & 10,6 \\
\hline 1988 & 8,1 & 3,6 & 32,9 & 5,1 & 36,1 & 6,0 & 20,1 & 4,3 & 34,4 & 5,5 \\
\hline 1989 & 4,6 & 1,8 & 32,9 & 9,4 & 41,1 & 10,9 & 18,4 & 5,5 & 36,7 & 10,1 \\
\hline 1990 & 7,5 & 2,4 & 24,1 & 3,1 & 38,3 & 5,4 & 15,6 & 2,7 & 30,6 & 4,2 \\
\hline 1991 & 5,2 & 3,0 & 37,5 & 4,9 & 46,5 & 4,7 & 21,2 & 4,0 & 41,7 & 4,8 \\
\hline 1992 & 7,1 & 0,0 & 25,3 & 6,1 & 41,9 & 6,0 & 16,2 & 3,1 & 33,0 & 6,1 \\
\hline
\end{tabular}

Fuente/Source:OPS. Sistema de Información Técnica

*Tasa/Rate 100.000 habitantes

M:Masculino/Male

F:Femenino/Female

Cuadro 15.c - Tasa* de mortalidad de adolescentes y jóvenes por suicidio, según edad y sexo Puerto Rico 1980-1992

Table 15.c - Mortality rate among adolescents and young adults due to suicide, by age and sex Puerto Rico 1980-1992

\begin{tabular}{cccccccccccc}
\hline Edad & \multicolumn{1}{c}{ 10 a 14 años } & \multicolumn{2}{c}{ 15 a 19 años } & \multicolumn{2}{c}{20 a 24 años } & \multicolumn{2}{c}{10 a 19 años } & \multicolumn{2}{c}{15 a 24 años } \\
\hline Años & M & F & \multicolumn{1}{c}{ M } & F & M & F & M & F & M & F \\
\hline 1980 & 0,6 & 0,6 & 10,1 & 1,8 & 18,5 & 2,8 & 5,3 & 1,2 & 13,7 & 2,2 \\
1981 & 0,0 & 0,0 & 7,8 & 1,2 & 9,9 & 2,1 & 3,8 & 0,6 & 8,7 & 1,6 \\
1982 & 0,6 & 0,0 & 3,7 & 0,6 & 17,6 & 2,7 & 2,1 & 0,3 & 10,0 & 1,6 \\
1983 & 0,6 & 0,0 & 3,7 & 0,6 & 19,1 & 2,0 & 2,1 & 0,3 & 10,9 & 1,3 \\
1984 & 0,0 & 0,6 & 6,3 & 0,6 & 9,6 & 1,9 & 3,1 & 0,6 & 7,9 & 1,3 \\
1985 & 1,2 & 0,6 & 7,0 & 1,9 & 11,4 & 0,0 & 4,0 & 1,3 & 9,2 & 1,0 \\
1986 & 0,6 & 0,0 & 1,9 & 0,0 & 10,1 & 1,9 & 1,2 & 0,0 & 5,9 & 1,0 \\
1987 & 0,6 & 0,0 & 5,0 & 1,3 & 11,6 & 2,0 & 2,7 & 0,6 & 8,2 & 1,6 \\
1988 & 1,7 & 0,6 & 6,8 & 1,3 & 14,6 & 1,3 & 4,2 & 0,9 & 10,5 & 1,3 \\
1989 & 0,6 & 0,6 & 3,7 & 1,3 & 11,3 & 0,7 & 2,1 & 0,9 & 7,2 & 1,0 \\
1990 & 1,2 & 0,6 & 4,2 & 1,2 & 19,1 & 2,0 & 2,7 & 0,9 & 11,1 & 1,6 \\
1991 & 0,6 & 0,0 & 7,7 & 0,6 & 18,1 & 0,7 & 4,1 & 0,3 & 12,5 & 0,6 \\
1992 & 0,0 & 0,0 & 8,2 & 0,0 & 11,5 & 3,3 & 4,1 & 0,0 & 9,7 & 1,6 \\
\hline
\end{tabular}

Fuente/Source:OPS. Sistema de Información Técnica

*Tasa/Rate $\times 100.000$ habitantes

M:Masculino/Male

F:Femenino/Female 
Cuadro 15.d - Tasa* de mortalidad de adolescentes y jóvenes por homicidio, según edad y sexo Puerto Rico 1980-1992

Table 15.d - Mortality rate among adolescents and young adults due to homicide, by age and sex Puerto Rico 1980-1992

\begin{tabular}{ccccccccccc}
\hline Edad & \multicolumn{1}{c}{10 a 14 años } & \multicolumn{2}{c}{ 15 a 19 años } & \multicolumn{2}{c}{20 a 24 años } & \multicolumn{2}{c}{10 a 19 años } & \multicolumn{2}{c}{15 a 24 años } \\
\hline Años & M & F & M & F & M & F & M & F & M & F \\
\hline 1980 & 1,7 & 0,0 & 33,7 & 2,4 & 60,0 & 6,3 & 17,5 & 1,2 & 45,2 & 4,2 \\
1981 & 0,6 & 2,4 & 39,5 & 3,6 & 71,8 & 5,5 & 19,8 & 3,0 & 53,7 & 4,5 \\
1982 & 1,8 & 0,0 & 32,3 & 3,0 & 57,4 & 8,8 & 16,7 & 1,5 & 43,7 & 5,8 \\
1983 & 2,4 & 0,6 & 19,3 & 5,6 & 55,3 & 6,0 & 10,6 & 3,1 & 36,1 & 5,8 \\
1984 & 0,0 & 0,6 & 31,0 & 5,7 & 45,2 & 5,8 & 15,0 & 3,1 & 37,8 & 5,8 \\
1985 & 2,4 & 1,9 & 36,3 & 3,2 & 58,4 & 4,5 & 18,8 & 2,5 & 47,1 & 3,8 \\
1986 & 3,6 & 1,2 & 43,9 & 7,7 & 64,4 & 9,7 & 23,1 & 4,4 & 53,9 & 8,7 \\
1987 & 1,2 & 0,0 & 24,5 & 1,3 & 54,4 & 5,9 & 12,5 & 0,6 & 38,9 & 3,5 \\
1989 & 1,2 & 0,0 & 30,4 & 4,4 & 72,2 & 11,3 & 15,3 & 2,2 & 50,2 & 7,8 \\
1989 & 1,7 & 0,0 & 29,9 & 2,5 & 56,0 & 9,5 & 15,4 & 1,2 & 42,0 & 5,9 \\
1990 & 1,2 & 0,0 & 47,6 & 2,5 & 70,2 & 10,9 & 23,9 & 1,2 & 58,0 & 6,5 \\
1991 & 3,5 & 1,2 & 75,0 & 7,4 & 100,0 & 11,5 & 38,8 & 4,3 & 86,5 & 9,3 \\
1992 & 3,6 & 2,5 & 65,3 & 4,3 & 125,7 & 8,7 & 34,5 & 3,4 & 93,4 & 6,4 \\
\hline
\end{tabular}

Fuente/Source:OPS. Sistema de Información Técnica

*Tasa/Rate x 100.000 habitantes

M:Masculino/Male

F:Femenino/Female

\section{TRINIDAD \& TOBAGO}

La mortalidad por causas externas en la población total disminuye progresivamente en un $12 \%$ en el período estudiado (19801994) y en un $22 \%$ entre los hombres de 15 a 24 años. Las mujeres de 15 a 24 años aumentan en un $27 \%$ las tasas entre los años extremos del período. El grupo de 10 a 14 años mantiene tasas estables, a pesar de sufrir oscilaciones durante el período estudiado (Cuadros 1.3 a 1.5 en Anexo y 16.a).

Los accidentes del tráfico y los suicidios determinan la mortalidad por causas externas en la población total de Trinidad \& Tobago, seguido por los homicidios. Las tasas de mortalidad por accidentes de tráfico disminuyen en un 45\% en la población total entre 1980 y 1994. En cambio, las tasas de mortalidad por homicidios se quintuplican en igual período y las tasas de suicidio se triplican en la misma población (Cuadros 1.6, 1.12 y 1.9 en Anexo).

En el grupo de adolescentes y jóvenes la tasa de mortalidad por homicidios es el componente principal de la mortalidad por causas externas, seguido muy cercanamente de suicidios y accidentes de tráfico.
La mortalidad por accidentes de tráfico en los adolescentes y jóvenes hombres tiende a una disminución, al igual que en la población total. El mayor descenso se observa en el grupo de 15 a 19 años (63\% de descenso entre 1980 y 1994) (Cuadro 16.b).

Las tasas de suicidio aumentan un $130 \%$ en los jóvenes hombres de 15 a 24 años y se triplican en las mujeres de 15 a 24 años, observándose el mayor ascenso en el grupo de mujeres de 15 a 19 años (Cuadro 16.c).

Las tasas de homicidio en Trinidad \& Tobago son bajas, pero han sufrido un aumento en los jóvenes hombres de 15 a 24 años, de tasas de 2,4 por 100.000 en 1980 a 21,4 por 100.000 en 1994. El número de homicidios en mujeres es escaso, por lo que no se pueden describir tendencias. Pero, son significativas las tasas de 8,5 para 100.000 mujeres de 15 a 19 años y de 10,7 por 100.000 para las del grupo etário de 20 a 24 años (Cuadro 16.d).

En suma, la disminución de las tasas de mortalidad por causas externas en adolescentes y jóvenes de Trinidad \& Tobago se explica por una disminución de las tasas de mortalidad por accidentes de tráfico. Hay tendencias francas de aumento de la mor- 
Cuadro 16.a - Tasa* de mortalidad de adolescentes y jóvenes por causas externas, según edad y sexo - Trinidad y Tobago 1980-1994

Table 16.a - Mortality rate among adolescents and young adults due to external causes, by age and sex-Trinidad y Tobago 1980-1994

\begin{tabular}{ccrcccccccc}
\hline Edad & \multicolumn{3}{c}{10 a 14 años } & \multicolumn{2}{c}{15 a 19 años } & \multicolumn{2}{c}{20 a 24 años } & \multicolumn{2}{c}{10 a 19 años } & \multicolumn{2}{c}{15 a 24 años } \\
\hline Años & M & \multicolumn{1}{c}{$\mathrm{F}$} & $\mathrm{M}$ & $\mathrm{F}$ & $\mathrm{M}$ & $\mathrm{F}$ & $\mathrm{M}$ & $\mathrm{F}$ & $\mathrm{M}$ & $\mathrm{F}$ \\
\hline 1980 & 16,4 & 6,6 & 77,6 & 19,4 & 122,8 & 23,2 & 48,4 & 13,3 & 98,4 & 21,1 \\
1981 & 16,7 & 5,1 & 80,6 & 22,7 & 88,1 & 27,6 & 50,4 & 14,4 & 84,1 & 25,0 \\
1982 & 20,0 & 3,4 & 96,9 & 29,2 & 91,8 & 28,3 & 60,0 & 16,9 & 94,4 & 28,8 \\
1983 & 35,0 & 16,9 & 88,9 & 20,6 & 127,4 & 27,4 & 62,6 & 18,9 & 108,0 & 24,0 \\
1984 & 36,7 & 11,9 & 80,3 & 32,8 & 142,9 & 22,2 & 58,7 & 22,5 & 114,9 & 28,1 \\
1985 & 13,3 & 3,4 & 78,0 & 20,3 & 109,4 & 21,9 & 45,4 & 11,9 & 95,1 & 21,5 \\
1986 & 11,5 & 6,8 & 58,6 & 22,4 & 117,5 & 28,6 & 34,5 & 14,5 & 90,0 & 26,1 \\
1987 & 14,8 & 3,4 & 67,2 & 10,3 & 139,3 & 30,6 & 40,3 & 6,8 & 104,2 & 20,8 \\
1988 & 19,7 & 13,6 & 72,4 & 13,8 & 106,8 & 21,7 & 45,4 & 13,7 & 78,9 & 15,7 \\
1989 & 22,6 & 15,0 & 69,5 & 24,1 & 101,8 & 34,5 & 45,5 & 19,5 & 72,8 & 25,0 \\
1990 & 21,9 & 11,3 & 67,8 & 19,0 & 105,4 & 33,3 & 43,9 & 15,0 & 83,9 & 25,6 \\
1991 & 12,1 & 26,6 & 69,5 & 15,5 & 147,3 & 23,2 & 39,2 & 21,3 & 50,4 & 18,8 \\
1992 & 23,2 & 6,0 & 63,3 & 15,5 & 120,0 & 23,2 & 41,9 & 10,4 & 90,4 & 19,3 \\
1993 & 15,3 & 12,9 & 60,0 & 16,9 & 132,1 & 21,4 & 35,6 & 14,7 & 94,0 & 19,3 \\
1994 & 17,3 & 5,5 & 55,7 & 27,1 & 101,8 & 26,8 & 34,6 & 15,2 & 76,5 & 26,7 \\
\hline
\end{tabular}

Fuente/Source:OPS. Sistema de Información Técnica

*Tasa/Rate x 100.000 habitantes

M:Masculino/Male

F: Femenino/Female

Cuadro 16.b - Tasa* de mortalidad de adolescentes y jóvenes por accidentes de vehículo a motor, según edad y sexo - Trinidad y Tobago 1980-1994

Table 16.b - Mortality rate among adolescents and young adults due to traffic accidents, by age and sex-Trinidad y Tobago 1980-1994

\begin{tabular}{ccccccccccc}
\hline Edad & \multicolumn{1}{c}{10 a 14 años } & \multicolumn{2}{c}{15 a 19 años } & \multicolumn{2}{c}{20 a 24 años } & \multicolumn{2}{c}{10 a 19 años } & \multicolumn{2}{c}{15 a 24 años } \\
\hline Años & M & \multicolumn{1}{c}{$\mathrm{F}$} & \multicolumn{1}{c}{ M } & $\mathrm{F}$ & $\mathrm{M}$ & $\mathrm{F}$ & $\mathrm{M}$ & $\mathrm{F}$ & $\mathrm{M}$ & $\mathrm{F}$ \\
\hline 1980 & 9,8 & 0,0 & 22,4 & 6,0 & 61,4 & 1,8 & 16,4 & 3,1 & 40,3 & 4,1 \\
1981 & 1,7 & 0,0 & 26,9 & 4,5 & 28,8 & 3,4 & 15,0 & 2,4 & 27,8 & 4,0 \\
1982 & 6,7 & 3,4 & 33,8 & 7,7 & 45,9 & 10,0 & 20,8 & 5,6 & 39,7 & 8,8 \\
1983 & 10,0 & 6,8 & 33,3 & 6,3 & 43,5 & 6,5 & 22,0 & 6,6 & 38,4 & 6,4 \\
1984 & 13,3 & 1,7 & 39,3 & 4,9 & 46,0 & 9,5 & 26,4 & 3,3 & 43,8 & 7,4 \\
1985 & 1,7 & 3,4 & 35,6 & 6,8 & 39,1 & 6,3 & 18,5 & 5,1 & 37,7 & 6,6 \\
1986 & 4,9 & 6,8 & 24,1 & 5,2 & 55,6 & 6,3 & 14,3 & 6,0 & 40,8 & 5,9 \\
1987 & 8,2 & 0,0 & 27,6 & 0,0 & 41,0 & 4,8 & 17,6 & 0,0 & 34,5 & 2,5 \\
1988 & 8,2 & 0,0 & 22,4 & 3,4 & 22,0 & 3,0 & 15,1 & 1,7 & 19,5 & 3,0 \\
1989 & 6,5 & 1,7 & 15,3 & 3,4 & 28,1 & 3,4 & 10,7 & 2,5 & 18,4 & 2,9 \\
1990 & 7,8 & 3,2 & 11,9 & 6,9 & 16,1 & 3,5 & 9,8 & 5,0 & 13,6 & 5,1 \\
1991 & 1,5 & 12,5 & 8,5 & 0,0 & 20,0 & 1,8 & 4,8 & 6,6 & 13,7 & 0,9 \\
1992 & 5,8 & 0,0 & 8,3 & 5,2 & 21,8 & 7,1 & 7,0 & 2,4 & 14,8 & 6,1 \\
1993 & 6,9 & 2,9 & 13,3 & 3,4 & 35,7 & 3,6 & 9,8 & 3,1 & 23,9 & 3,5 \\
1994 & 4,0 & 1,4 & 8,2 & 1,7 & 26,8 & 7,1 & 5,9 & 1,5 & 16,8 & 4,3 \\
\hline
\end{tabular}

Fuente/Source:OPS. Sistema de Información Técnica

*Tasa/Rate x 100.000 habitantes

M:Masculino/Male

F: Femenino/Female 
Cuadro 16.c - Tasa* de mortalidad de adolescentes y jóvenes por suicidio, según edad y sexo Trinidad y Tobago 1980-1994

Table 16.c - Mortality rate among adolescents and young adults due to suicide, by age and sex Trinidad y Tobago 1980-1994

\begin{tabular}{|c|c|c|c|c|c|c|c|c|c|c|}
\hline \multirow{2}{*}{$\frac{\text { Edad }}{\text { Años }}$} & \multicolumn{2}{|c|}{10 a 14 años } & \multicolumn{2}{|c|}{15 a 19 años } & \multicolumn{2}{|c|}{20 a 24 años } & \multicolumn{2}{|c|}{10 a 19 años } & \multicolumn{2}{|c|}{15 a 24 años } \\
\hline & $M$ & $\mathrm{~F}$ & M & $\mathrm{F}$ & $M$ & $\mathrm{~F}$ & $M$ & $\mathrm{~F}$ & $M$ & $\mathrm{~F}$ \\
\hline 1980 & 0,0 & 1,6 & 6,0 & 1,5 & 8,8 & 3,6 & 3,1 & 1,6 & 7,3 & 2,4 \\
\hline 1981 & 0,0 & 1,7 & 9,0 & 6,1 & 13,6 & 5,2 & 4,7 & 4,0 & 11,1 & 5,6 \\
\hline 1982 & 3,3 & 0,0 & 6,2 & 10,8 & 9,8 & 6,7 & 4,8 & 5,6 & 7,9 & 8,8 \\
\hline 1983 & 0,0 & 1,7 & 6,3 & 12,7 & 3,3 & 7,4 & 6,3 & 12,7 & 6,4 & 13,6 \\
\hline 1984 & 1,7 & 1,7 & 13,1 & 14,8 & 20,6 & 0,0 & 7,4 & 8,3 & 17,4 & 7,4 \\
\hline 1985 & 1,7 & 0,0 & 3,4 & 1,7 & 3,1 & 3,1 & 2,5 & 0,8 & 3,3 & 2,5 \\
\hline 1986 & 1,6 & 0,0 & 10,3 & 6,9 & 11,1 & 12,7 & 5,9 & 3,4 & 10,8 & 10,1 \\
\hline 1987 & 1,6 & 1,7 & 12,1 & 5,2 & 29,5 & 8,1 & 6,7 & 3,4 & 21,0 & 6,7 \\
\hline 1988 & 0,0 & 6,8 & 10,3 & 6,9 & 27,1 & 10,0 & 5,0 & 6,8 & 16,5 & 7,5 \\
\hline 1989 & 4,8 & 3,3 & 22,0 & 15,5 & 29,8 & 19,0 & 13,2 & 9,3 & 22,1 & 14,7 \\
\hline 1990 & 1,6 & 4,8 & 15,3 & 8,6 & 37,5 & 21,1 & 8,1 & 6,7 & 25,4 & 14,5 \\
\hline 1991 & 1,5 & 6,3 & 16,9 & 8,6 & 58,2 & 10,7 & 8,8 & 7,4 & 35,9 & 9,4 \\
\hline 1992 & 2,9 & 4,5 & 16,7 & 6,9 & 38,2 & 8,9 & 9,3 & 5,6 & 27,0 & 7,9 \\
\hline 1993 & 0,0 & 2,9 & 10,0 & 3,4 & 35,7 & 12,5 & 4,5 & 3,1 & 22,2 & 7,9 \\
\hline 1994 & 2,7 & 0,0 & 9,8 & 10,2 & 25,0 & 7.1 & 5,9 & 4,5 & 16,8 & 8,6 \\
\hline
\end{tabular}

Fuente/Source:OPS. Sistema de Información Técnica

*Tasa/Rate x 100.000 habitantes

M:Masculino/Male

F:Femenino/Female

Cuadro 16.d - Tasa* de mortalidad de adolescentes y jóvenes por homicidio, según edad y sexo Trinidad y Tobago 1980-1994

Table 16.d - Mortality rate among adolescents and young adults due to homicide, by age and sex Trinidad y Tobago 1980-1994

\begin{tabular}{|c|c|c|c|c|c|c|c|c|c|c|}
\hline \multirow{2}{*}{$\frac{\text { Edad }}{\text { Años }}$} & \multicolumn{2}{|c|}{10 a 14 años } & \multicolumn{2}{|c|}{15 a 19 años } & \multicolumn{2}{|c|}{20 a 24 años } & \multicolumn{2}{|c|}{10 a 19 años } & \multicolumn{2}{|c|}{15 a 24 años } \\
\hline & $M$ & $\mathrm{~F}$ & $M$ & $\mathrm{~F}$ & $M$ & $\mathrm{~F}$ & $M$ & $\mathrm{~F}$ & $M$ & $\mathrm{~F}$ \\
\hline 1980 & 0,0 & 0,0 & 3,0 & 0,0 & 1,8 & 5,4 & 1,6 & 0,0 & 2,4 & 2,4 \\
\hline 1981 & 0,0 & 0,0 & 3,0 & 0,0 & 6,8 & 3,4 & 1,6 & 0,0 & 4,8 & 1,6 \\
\hline 1982 & 0,0 & 0,0 & 6,2 & 0,0 & 6,6 & 1,7 & 3,2 & 0,0 & 6,3 & 0,8 \\
\hline 1983 & 0,0 & 1,7 & 6,3 & 0,0 & 16,1 & 3,2 & 3,3 & 0,8 & 11,2 & 2,4 \\
\hline 1984 & 1,7 & 0,0 & 4,9 & 1,6 & 15,9 & 1,6 & 3,3 & 0,8 & 10,7 & 1,7 \\
\hline 1985 & 1,7 & 0,0 & 13,6 & 6,8 & 20,3 & 4,7 & 7,6 & 3,4 & 17,2 & 5,8 \\
\hline 1986 & 1,6 & 0,0 & 3,4 & 3,4 & 14,3 & 3,2 & 2,5 & 1,7 & 9,2 & 3,4 \\
\hline 1987 & 0,0 & 1,7 & 8,6 & 3,4 & 23,0 & 8,1 & 4,2 & 2,6 & 16,0 & 5,8 \\
\hline 1988 & 0,0 & 0,0 & 5,2 & 0,0 & 25,4 & 3,3 & 2,5 & 0,0 & 13,5 & 1,5 \\
\hline 1989 & 1,6 & 8,3 & 16,9 & 1,7 & 26,3 & 6,9 & 9,1 & 5,1 & 18,4 & 3,7 \\
\hline 1990 & 0,0 & 0,0 & 13,6 & 0,0 & 32,1 & 5,3 & 6,5 & 0,0 & 22,0 & 2,6 \\
\hline 1991 & 0,0 & 1,6 & 5,1 & 1,7 & 47,3 & 0,0 & 2,4 & 1,6 & 24,8 & 0,9 \\
\hline 1992 & 1,4 & 0,0 & 10,0 & 1,7 & 34,5 & 5,4 & 5,4 & 0,8 & 21,7 & 3,5 \\
\hline 1993 & 0,0 & 2,9 & 6,7 & 3,4 & 37,5 & 1,8 & 3,0 & 3,1 & 21,4 & 2,6 \\
\hline 1994 & 1,3 & 0,0 & 11,5 & 8,5 & 33,9 & 10,7 & 5,9 & 3,8 & 21,4 & 2,6 \\
\hline
\end{tabular}

Fuente/Source:OPS. Sistema de Información Técnica

*Tasa/Rate x 100.000 habitantes

M:Masculino/Male

F:Femenino/Female 
talidad por suicidio y homicidio entre los jóvenes de 15 a 24 años en el período estudiado.

\section{Discusion y Conclusiones}

\section{Tendencia de las tasas de mortalidad por causas externas en la Región de las Américas}

Las conclusiones de este trabajo se pueden extrapolar con precaución a la Región de las Américas. Se analizan 16 países que cuentan con población e información suficiente para cumplir los requisitos mínimos necesarios para el análisis estadístico de este trabajo. Estos 16 países representan un 90\% de la población de la Región de las Américas.

\section{A. Mortalidad por causas externas}

La mortalidad por causas externas en la población total y adolescente, entre los años 1980 y 1994, último año com datos disponibles en la mayor parte de los paises en la Región de las Américas, continúa la tendencia descendente previamente observada en 1990 y descrita por Yunes y Rajs ${ }^{7}$. Las tasas en 8 de los países estudiados presentan francas tendencias decrecientes de la mortalidad por causas externas.

Los países que presentan tasas decrecientes, tanto en la población total como adolescente y joven, en el período estudiado son: Canadá, EEUU, Ecuador, México, Chile, Costa Rica, Trinidad \& Tobago y El Salvador (no se dispone de datos intermedios de este último). Venezuela y Argentina, que hasta el año 1990 presentaban también tasas decrecientes, han sufrido un aumento de las tasas de mortalidad por causas externas en estos últimos cuatro años en la población total y adolescente. En estos dos países, junto con Uruguay, Panamá y Cuba se observa descenso de las tasas en alguno de los grupos, sea población total o algún grupo de adolescentes y/o jóvenes. En Cuba, a pesar que las tasas han aumentado para la población total y los jóvenes varones, hay franca disminución en las mujeres.
Colombia y Brasil son los únicos países analizados que continúan con tendencias francamente ascendentes en las tasas de mortalidad por causa externa en la población total y en todos los grupos de adolescentes y jóvenes por edad y sexo entre 1980 y 1995.

Respecto a la distribución por sexo, las tasas de mortalidad por causas externas son significativamente mayores en hombres, con cuocientes hombre/mujer entre $2 / 1$ a 10/1. La diferencia es mayor a favor de los hombres en países que presentan mayores tasas de mortalidad por causas externas como Colombia, El Salvador y Brasil.

Respecto a la magnitud de las tasas de mortalidad, los países se pueden clasificar como de alta, mediana y baja mortalidad por causas externas, en relación al último año estudiado. La interpretación de este tipo de análisis comparativo puede estar sujeta a error ya que se comparan tasas registradas de mortalidad de cada país, sabiendo que existe un porcentaje de subregistro que difiere en cada país, grupo etáreo y período estudiado. En este caso la posibilidad de error se minimiza, ya que los países con mayor porcentaje de subregistro son aquellos con tasas mas altas de mortalidad por causas externas (Colombia, El Salvador, Brasil y Venezuela).

Los países con tasas elevadas de mortalidad por causas externas en la población total (mayores de 70 por 100.000) son, en orden decreciente: Colombia, El Salvador, Cuba, Venezuela, Puerto Rico y Brasil. Tasas moderadas de mortalidad por causas externas (entre 50 y 70 por 100.000): México, Ecuador, Chile, Uruguay, EEUU, Panamá y Argentina (puede haber sesgo en la clasificación de Ecuador y Panamá por presentar subregistros cercanos al 20\%). Canadá y Costa Rica presentan tasas bajas de mortalidad por causas externas, con cifras menores de 50 por 100.000 .

Respecto a las tasas de mortalidad por causas externas se tomará como referencia la tasa de mortalidad del último año disponible del grupo mas afectado: varones de 15 a 24 años. Los países de alta mortalidad por causas externas (tasas mayores de 150 por 
100.000) en este grupo de edad son, en orden decreciente: Colombia, El Salvador, Venezuela, Brasil y Puerto Rico. Los países de mortalidad intermedia (tasas entre $100 \mathrm{y}$ 150 por 100.000) son: México, Ecuador, Panamá y EEUU. Los países de mortalidad baja (menor de 100 por 100.000) son: Cuba, Chile, Argentina, Cuba, Trinidad \& Tobago, Uruguay, Costa Rica y Canadá. Las mismas observaciones respecto a la posibilidad de error por subregistro deben considerarse en este caso.

La diferencia de sexo, en el grupo de adolescentes y jóvenes fluctúa entre un cuociente hombre/mujer de 2/ 1 en Cuba a 9/1 en Colombia.

\section{B. Mortalidad por subgrupos de causas externas.}

Las tasas de mortalidad por subgrupos de causas externas presentan variaciones entre los países estudiados. En la población total su determinante principal son los accidentes de tráfico en todos los países estudiados, excepto: El Salvador y Colombia, en que la causa principal son los homicidios; Cuba, en que predominan los suicidios; Panamá el año 1989, en que predominan los homicidios por el conflicto bélico; México en que, a partir de 1984, predomina el homicidio y Brasil en que también predomina el homicidio a partir de 1991.

En adolescentes y jóvenes el homicidio juega un rol determinante: En México, Puerto Rico y Argentina el determinante principal de la mortalidad por causas externas es el homicidio, a diferencia de la población total de estos países, donde predominan los accidentes de tráfico. En Colombia, El Salvador y Brasil (desde el año 1991) el homicidio es la primera causa externa de mortalidad tanto en la población total como en jóvenes.

- Mortalidad por accidentes de tráfico: magnitud y tendencias

Venezuela, Brasil, Puerto Rico, El Salvador y Colombia son los países con mayores tasas de mortalidad por accidentes de tráfico en la población total.(Tasas mayores de 17 por 100.000).

Tomando como referencia al grupo de adolescentes hombres de 15 a 24 años, los países con mayores tasas de mortalidad por accidentes de tráfico en este grupo etáreo son: Panamá, El Salvador, EEUU, Venezuela, Brasil, y Puerto Rico con tasas mayores a 30 por 100.000. Colombia, Canadá, Cuba, Costa Rica, México, Ecuador y Argentina tienen tasas de 20 a 30 por 100.000 . Uruguay, Chile y Trinidad \& Tobago tienen tasas bajas, menores de 20 por 100.000 .

Las tasas en adolescentes son mayores en hombres, con cuocientes hombre/mujer entre $2 / 1$ a $6 / 1$, las mayores diferencias se observan en países en que aún hay menor acceso de la mujer a manejar vehículos como en Ecuador, Puerto Rico, El Salvador y México.

A pesar que los accidentes de tráfico son la primera causa externa de mortalidad, llama la atención que las tasas de mortalidad por esta causa muestra tendencias francas a la disminución en la mayor parte de los países de la Región. Algunas excepciones son Chile y Uruguay, donde hay una tendencia estable en la población total, una tendencia a la disminución en los hombres de 15 a 24 años y una tendencia al aumento entre las mujeres de 15 a 24 años en el período estudiado. Este aumento puede explicarse también debido a un mayor acceso de las mujeres a manejar vehículos. En Colombia la mortalidad por accidentes de tráfico son estables en la población total y hay un leve aumento en adolescentes y jóvenes.

\section{- Mortalidad por suicidio: Magnitud y ten- dencias}

Las tasas de mortalidad por suicidio en general son mas elevadas para la población total que en adolescentes y jóvenes y son mas elevadas en sexo masculino y a mayor edad. Una excepción es Cuba en que las tasas de mortalidad por suicidio entre adolescentes y jóvenes son mayores en mujeres (1992 - 1995). 
Los países con mayores tasas de mortalidad por suicidio entre la población total son: Cuba, Canadá, Trinidad \& Tobago, Estados Unidos y Uruguay (en orden decreciente).

Tomando como referencia al grupo de hombres de 15 a 24 años de edad, los países con mortalidad por suicidio alta ( tasas mayores a 12 por 100.000) son, en orden decreciente: Canadá, EEUU, El Salvador, Trinidad \& Tobago, Cuba y Uruguay. Los países con tasas moderadas (entre 8 y 12 por 100.000): Costa Rica, Chile, Ecuador, Venezuela, Puerto Rico, Panamá y Argentina. Colombia, México y Brasil son los países con las tasas más bajas (menores de 8 por 100.000).

La tendencia de la mortalidad por suicidio en la mayor parte de los países es estacionaria excepto en: Panamá, México y Trinidad \& Tobago, en que hay aumento de las tasas en población total y adulto joven. En Canadá, Ecuador y Argentina la mortalidad por suicidio está aumentando principalmente entre los jóvenes. En Brasil las tasas están aumentando en el grupo de hombres de 15 a 24 años, especialmente a partir de 1989. En Cuba el suicidio es el primer factor de la mortalidad por causas externas en la población total y en las mujeres del mismo grupo etario.

- Mortalidad por homicidios: Magnitud y tendencias

Las tasas de mortalidad por homicidio en general son mas elevadas entre adolescentes y jóvenes que en la población total. Las tasas aumentan a medida que aumenta la edad en el grupo de 10 a 24 años y son mayores en hombres. El cuociente hombre/ mujer es muy variable y aumenta en la medida que aumenta la tasa de homicidios. Los mayores cuocientes los presentan El Salvador, Colombia y Puerto Rico.

Los países con mayores tasas de mortalidad por homicidios en la población total, en orden decreciente son: Colombia, El Salvador, Puerto Rico, Brasil y Venezuela.

Tomando como referencia al grupo de jóvenes varones de 15 a 24 años se pueden agrupar los países en categorías alta, moderada y baja de homicidios. Países de alta mortalidad (tasas mayores de 50 por 100.000) son, en orden decreciente: Colombia, El Salvador, Puerto Rico, Venezuela y Brasil. Países con moderada mortalidad por homicidio ( tasas entre 10 y 50 por 100.000) son : México, Ecuador Panamá, EEUU, Trinidad \& Tobago, Cuba y Argentina. Países con baja mortalidad por homicidio (tasas menores de 10 por 100.000) son: Costa Rica, Chile, Uruguay y Canadá.

Llama la atención que en la mayor parte de los países analizados existe una tendencia creciente en las tasas de mortalidad por homicidio en el período estudiado. En 9 países las tasas de homicidio se han incrementado tanto en la población total como en adolescentes y jóvenes: Puerto Rico, Trinidad \& Tobago, Argentina, Uruguay, Panamá, Costa Rica, Venezuela, Ecuador y Brasil. ( se excluyen de este análisis El Salvador y Cuba ya que cuentan con información limitada).

En México hay una leve tendencia a la disminución del homicidio en la población total y entre los adolescentes de 15 a 19 años. En Chile hay niveles estacionarios en la población total y una disminución en el grupo de 15 a 24 años. En Canadá la tasa es descendente en la población total y en los adolescentes y los jóvenes.

En Colombia se observa un descenso en la mortalidad por homicidios en los adolescentes y los jovenes, de ambos los sexos, en el año de 1995, último año disponible en esta análisis.

En resumen, el mayor determinante de la mortalidad por causas externas tanto en la población total como en adolescentes y jóvenes son los accidentes de tráfico, que muestran tendencias decrecientes en casi todos los países de la Región de las Américas.

La mortalidad por homicidios es un problema creciente en la región. La tendencia en 9 de los países estudiados es al ascenso progresivo de las tasas de mortalidad por esta causa tanto en la población total como en adolescentes y jóvenes. 
En El Salvador, Colombia y México el homicidio es el primer determinante de la mortalidad por causas externas en la población total. El homicidio es el factor determinante de la mortalidad por causas externas entre adolescentes y jóvenes en estos tres países, además de: Brasil y Puerto Rico.

A pesar que las tasas mas altas de homicidio entre adolescentes y jóvenes la presentan los jóvenes varones de 20 a 24 años, hay un aumento alarmante en el grupo de hombres de 15 a 19 años especialmente en Brasil.

Las tasas de mortalidad por suicidio son en general mas bajas en los adolescentes que en la población total, sin embargo, constituye una causa de mortalidad considerable entre adolescentes y jóvenes de Cuba, Canadá, EEUU, Trinidad \& Tobago, Argentina y El Salvador.

Los resultados de este análisis revelan que la violencia y sus manifestaciones son un problema creciente en la Región de las Américas, principalmente a partir de los años noventa. Las tasas crecientes de la mortalidad por homicidios reflejan la nece- sidad inminente de programas de prevención de violencia en la Región, principalmente destinados a adolescentes y jóvenes. La contribución importante del sector salud será implementar Sistemas de Vigilancia Epidemiológica con el objetivo de conocer mejor el perfil de la violencia tanto como sus factores determinantes, con objeto de contribuir al desarrollo de un Plan de Acción Regional para la prevención de la violencia.

\section{Agradecimientos}

Los autores agradecen la ayuda parcial para el financiamiento de este trabajo al CNPq. - Brasil (Proceso n 520.114/98-1). A la Organización Panamericana de La Salud - OPS/ Programa Especial de Análisis de Salud, que permitieron el aceso a los datos. A la Analista de Proyectos Aparecida Vieira de Melo, de la Fundação Seade / Secretaria de Estado da Saúde de São Paulo Coordenação dos Institutos de Pesquisa (S.E.S/CIP), por la revisión del texto.

\section{Summary}

During the last few decades, there has been a considerable increase in the level of violence throughout the world. Violence has reached epidemic proportions, becoming one of the most serious public health problems faced by the Region of the Americas. In fact, mortality rates from external causes (homicides, suicides, traffic accidents and other injuries) began to affect the general mortality rate significantly. The situation is particularly alarming among adolescents and young people, between 10 and 24 years of age. Of all homicides reported in the Region of the Americas in 1990, 28.7\% correspond to young people. In 10 of the 21 countries of the Region with population greater than one million, homicide is the secondleading cause of death in the 15 to 24 year age group, and one of the five lead- ing causes of death in 17 of the 21 countries. Adolescents are one of the populations most affected by violence in all of its forms and they are not only victims, but also perpetrators or intermediaries of various acts of violence. Adolescents and young people in the Region of the Americas are exposed to a great deal of violence portrayed in the media. The are few published studies regarding the magnitude of violence in American countries. The objective of this paper is to describe mortality trends from external causes or violence in this region in the total population, among adolescents, and young people (10 to 24 years of age) from 1980 until the last year in the 90's in which data was available for each country. The causes of death have been classified according to the International Classification of Diseases, 
Ninth Revision (ICD-9). The countries that fulfilled a reasonable criteria of quality of data were: Costa Rica, El Salvador, Panama (Central America); USA, Canada, Mexico (North America): Colombia, Ecuador, Venezuela, Chile, Argentina, Uruguay, Brazil (Latin America): Cuba, Puerto Rico, Trinidad and Tobago (Caribbean). These 16 countries represent $90 \%$ of the population of the Americas. Mortality rates specific for age and sex were analyzed in the following age groups: all ages, 10 to 14, 15 to 19, 20 to 24, 10 to 19, and 15 to 24. Mortality from external causes for the total population in the Region continues on its downward trend. Colombia and Brazil are the only countries analyzed in whichrates continued to increase in the total population, among adolescents, and young people between 1980 and 1995 (tables 1.3; 6.a; 9.a). With respect to distribution by sex, mortality rates from external causes are significantly greater among males than among females, with a male/female ratio from 2:1 to 10:1. The greatest differences in the rates between sexes are observed in Colombia, El Salvador and Brazil (tables 1.4; 1.5). The countries with the highest mortality rates from external causes in the total population (greater than 70 per 100,000) are in decreasing order: Colombia, El Salvador, Cuba, Venezuela, Puerto Rico and Brazil (table 1.3). For comparative purposes, mortality rates from external causes for males between 15 and 24 years of age will be used as a reference for comparisons among countries. The countries with the highest mortality rates from external causes (rates greater than 150 per 100,000) are in decreasing order: Colombia, El Salvador, Venezuela, Puerto Rico and Brazil (tables 7.a; 3.a; 8.a; 15.a; 13.a). The ratio between male and female adolescents and young people varies between 2:1 in Cuba to 9:1 in Colombia (tables 14.a; 7.a). The countries with the highest mortality rates from traffic accidents in the total population, with rates greater than 17 per 100,000 are Venezuela, Brazil, Puerto Rico, El Salvador and Colombia (table 1.6). Utilizing males in the 15-24 age group as the point of reference, thecountries with the highest mortality rates from traffic accidents are: Panama, El Salvador, USA, Venezuela, Brazil and Puerto Rico, with rates greater than 30 per 100,000 (tables 1.b; 3.b; 5.b; 8.b; 13.b; 15.b). The rates are greater among males than females, with ratios varying from 2:1 (USA: table 5.b) to 5:1 (Puerto Rico: table 15.b). Although traffic accidents represent the largest contributing factor to mortality rates from $\mathrm{ex}$ ternal causes, it should be noted that there has been a significant decline in the rates of most countries throughout the Region. Suicide rates in general tend to be greater for the total population than for adolescents and young people, and are higher in males and in the elderly. One exception, however, is Cuba, where suicide rates among adolescents and young people were highest in the female population for the period 1992 1995 (table 14.c). The countries with the highest suicide rates in the total population are in decreasing order: Cuba, Canada, Trinidad and Tobago, United States and Uruguay (table 1.9). Using males from 15 to 24 as the reference group, the countries with the highest suicide rates (rates greater than 12 per 100,000) are, in decreasing order: Canada, USA, El Salvador, Trinidad and Tobago, Cuba and Uruguay (tables: 4.c; 5.c; 3.c; 16.c; 14.c; 12.c). Homicide rates in general are greater among adolescents and young people than in the total population. Homicide rates are greater in males than in females, and increase with age within the 10 to 24 year age group. The ratio between sexes is quite varied, and increases parallel with increases in homicides. The greatest ratios exist in El Salvador, Colombia and Puerto Rico (tables 3.d; 7.d; 15.d). The countries with the highest homicide rates in the total population, in decreasing order, are: Colombia, El Salvador, Puerto Rico, Brazil, and Venezuela. Colombia's rate is three times that of El Salvador (table 1.12). Using males between 15 and 24 as a reference group, the countries analyzed with a high homicide rate (greater than 50 per 100.000), in decreasing order are: Colombia, El Sal- 
vador, Puerto Rico, Brazil and Venezuela (tables 7.d; 3.d; 15.d; 13.d; 8.d). Results from this analysis reveal that violence and its manifestations are a growing problem for the Region of the Americas. The increasing homicide rates reflect the need for violence prevention programs in the Region targeting adolescents and young people. Given its multi-factorial nature, violence should be prevented through the coordinated and ac- tive participation of all sectors of society, with a multidisciplinary and inter-sectorial focus. The health sector can contribute to the development of the Regional Plan of Action for Violence Prevention by implementing epidemiological surveillance systems with the purpose of providing information to better understand trends in violence in the Region, as well as risk factors.

\section{Referencias}

1. American Academy of Pediatrics. Committee on Communications. Media violence. Pediatrics 1995; 95: 949-51.

2. Eron L D, Gentry J H, Schlege P. Reason to hope: a psychosocial perspective on violence and youth. Washington (DC): American Psychological Association; 1994.

3. Maddaleno M, Munist M, Serrano C V, Silber TJ, Yunes J, organizadores). La salud del adolescente y del joven. Washington (DC): Organización Panamericana de la Salud; 1995. (OPS-Publicación Científica, 552).

4. Organización Panamericana de la Salud. Programa Especial de Análisis de Salud. Situación de salud en las Américas: indicadores básicos 1999. Washington (DC); 1999. (OPS/SHA/99.01).
5. Organización Panamericana de la Salud. Memorias de la Conferencia Interamericana sobre "Sociedad, Violencia y Salud”; 1994 nov; Washington (DC): OPS; 1994.

6. Organización Panamericana de la Salud. Las condiciones de salud en las Américas. Washington (DC); 1994. v. 1 (OPS - Publicación Científica, 549).

7. Yunes J. Mortalidad por causas violentas en la Región de las Américas. Bol Oficina Sanit Panam 1993; 114: 302-16.

8. Yunes J, Rajs D. Tendencia de la mortalidad por causas externas en la población general y entre adolescentes y jóvenes de la Región de las Américas. Cad Saúde Pública 1994; 10 Supl 1: 85-125. 


\section{Anexos/Annex}


Cuadro 1 - Tasa* de mortalidad por todas las causas. Región de las Américas - 1980-1997. Toda la población Table 1 - Mortality rate due to all causes. Region of the America - 1980-1997. Entire population.

\begin{tabular}{|c|c|c|c|c|c|c|c|c|c|c|c|c|c|c|c|c|c|c|}
\hline País/Año & 1980 & 1981 & 1982 & 1983 & 1984 & 1985 & 1986 & 1987 & 1988 & 1989 & 1990 & 1991 & 1992 & 1993 & 1994 & 1995 & 1996 & 1997 \\
\hline Argentina & 857,7 & 847,5 & 804,1 & 854,0 & 855,5 & 796,0 & 783,1 & 800,3 & 804,9 & 785,6 & 797,9 & 775,3 & 785,8 & 791,3 & 750,1 & 773,7 & 763 & $\ldots$ \\
\hline Brasil & 619,0 & 604,9 & 585,0 & 595,3 & 612,1 & 583,7 & 589,1 & 569,5 & 582,9 & 559,5 & 552,4 & 534,8 & 541,7 & 566,9 & 564,9 & 561,0 & $\ldots$ & $\ldots$ \\
\hline Canadá & 697,2 & 688,3 & 695,0 & 688,1 & 685,5 & 699,0 & 701,0 & 694,0 & 702,9 & 696,5 & 690,8 & 694,8 & 689,9 & 711,1 & 710,6 & 711,5 & $\ldots$ & $\ldots$ \\
\hline Chile & 661,6 & 617,4 & 607,9 & 635,9 & 628,7 & 608,9 & 587,9 & 564,7 & 585,5 & 583,5 & 596,3 & 559,6 & 544,8 & 553,7 & 539,1 & $\ldots$ & $\ldots$ & $\ldots$ \\
\hline Colombia & $\ldots$ & 617,1 & $\ldots$ & $\ldots$ & 442,4 & 486,3 & 453,0 & 461,0 & 455,3 & 451,1 & 442,3 & 459,0 & 461,3 & 454,8 & 445,9 & 440,8 & $\ldots$ & $\ldots$ \\
\hline Costa Rica & 406,0 & 382,1 & 378,4 & 378,0 & 386,9 & 397,2 & 384,3 & 382,1 & 380,5 & 381,5 & 374,6 & 378,9 & 377,7 & 374,7 & 385,6 & 395,7 & $\cdots$ & $\ldots$ \\
\hline Cuba & 574,7 & 592,3 & 570,4 & 587,4 & 597,1 & 637,7 & 619,4 & 632,2 & 653,6 & 641,6 & 680,7 & 670,6 & 699,5 & 722,1 & 717,6 & 710,8 & 723,0 & $\ldots$ \\
\hline Ecuador & 716,2 & 670,9 & 630,4 & 639,1 & 599,0 & 562,0 & 546,2 & 539,3 & 538,4 & 515,9 & 489,3 & 507,8 & 497,5 & 477,7 & 456,0 & 443,9 & 447,0 & $\ldots$ \\
\hline El Salvador & $\ldots$ & 818,9 & 712,6 & 695,8 & 610,1 & $\cdots$ & $\ldots$ & $\cdots$ & $\cdots$ & $\ldots$ & 552,3 & 513,2 & 525,0 & 530,1 & $\cdots$ & $\cdots$ & $\cdots$ & $\cdots$ \\
\hline México & 647,9 & 617,4 & 585,8 & 573,5 & 556,4 & 548,2 & 517,7 & 514,6 & 510,7 & 512,0 & 500,3 & 476,1 & 464,7 & 462,5 & 467,9 & 472,1 & $\ldots$ & $\ldots$ \\
\hline Panamá & 408,4 & 400,2 & 399,7 & 408,6 & 388,6 & 414,9 & 404,2 & 403,2 & 407,2 & 406,7 & $\ldots$ & $\cdots$ & $\cdots$ & $\ldots$ & $\ldots$ & $\ldots$ & 418,3 & 448,9 \\
\hline Puerto Rico & 636,7 & 650,1 & 654,3 & 647,7 & 651,6 & 688,8 & 688,2 & 697,6 & 724,7 & 742,6 & 740,5 & 739,3 & 763,4 & $\cdots$ & $\ldots$ & $\cdots$ & $\ldots$ & $\cdots$ \\
\hline Trinidad y Tobago & 693,7 & 670,4 & 686,5 & 668,4 & 682,9 & 691,9 & 654,1 & 675,7 & 665,8 & 672,1 & 663,1 & 654,8 & 674,5 & 689,1 & 717,0 & $\ldots$ & $\ldots$ & $\ldots$ \\
\hline Estados Unidos & 873,7 & 860,1 & 850,8 & 862,1 & 863,0 & 874,9 & 874,9 & 874,3 & 884,5 & 869,0 & 859,6 & 859,2 & 852,5 & 879,5 & 874,4 & 865,9 & 859,1 & 851,5 \\
\hline Uruguay & 1024,2 & 942,5 & 920,9 & 958,4 & 1003,7 & 949,6 & 951,5 & 981,7 & 1010,2 & 962,9 & 976,9 & $\cdots$ & $\cdots$ & $\cdots$ & $\cdots$ & $\cdots$ & $\cdots$ & $\cdots$ \\
\hline Venezuela & 509,1 & 517,8 & 492,0 & 470,3 & $\ldots$ & 463,0 & 446,0 & 448,6 & 440,3 & 446,3 & 461,9 & $\ldots$ & 448,0 & 446,7 & 463,1 & $\ldots$ & $\ldots$ & $\ldots$ \\
\hline
\end{tabular}

Fuente/Source: OPS. Sistema de Información Técnica

${ }^{*}$ Tasa/Rate x 100.000 habitantes 
Cuadro 1.1 - Tasa* de mortalidad por todas las causas. Región de las Américas - 1980-1997.Toda la población masculina

Table 1.1 - Mortality rate due to all causes. Region of the America - 1980-1997. Male population.

\begin{tabular}{|c|c|c|c|c|c|c|c|c|c|c|c|c|c|c|c|c|c|c|}
\hline País/Año & 1980 & 1981 & 1982 & 1983 & 1984 & 1985 & 1986 & 1987 & 1988 & 1989 & 1990 & 1991 & 1992 & 1993 & 1994 & 1995 & 1996 & 1997 \\
\hline Argentina & 988,6 & 983,9 & 923,4 & 976,4 & 973,0 & 893,2 & 883,3 & 902,1 & 903,3 & 886,7 & 891,8 & 873,2 & 881,4 & 887,9 & 841,4 & 862,5 & 857,8 & $\ldots$ \\
\hline Brasil & 711,4 & 698,9 & 681,4 & 696,2 & 717,7 & 685,2 & 693,6 & 668,9 & 685,7 & 664,8 & 655,6 & 636,5 & 644,2 & 672,2 & 667,8 & 660,8 & $\ldots$ & $\ldots$ \\
\hline Canadá & 796,1 & 784,3 & 785,1 & 775,0 & 768,0 & 779,4 & 773,6 & 765,4 & 776,0 & 765,4 & 754,4 & 755,7 & 749,9 & 766,3 & 760,2 & 759,0 & $\ldots$ & $\ldots$ \\
\hline Chile & 738,6 & 693,5 & 690,8 & 731,7 & 722,8 & 683,9 & 658,9 & 617,5 & 646,9 & 657,7 & 671,7 & 626,4 & 599,7 & 612,5 & 599,1 & $\ldots$ & $\ldots$ & $\ldots$ \\
\hline Colombia & $\ldots$ & 702,5 & $\ldots$ & $\ldots$ & 507,3 & 562,3 & 533,1 & 545,3 & 544,2 & 543,1 & 536,2 & 567,8 & 571,2 & 561,5 & 548,8 & 536,6 & $\ldots$ & $\ldots$ \\
\hline Costa Rica & 475,4 & 427,9 & 427,3 & 421,7 & 432,2 & 445,7 & 428,3 & 428,9 & 429,0 & 431,5 & 425,7 & 422,8 & 432,6 & 420,3 & 435,8 & 447,1 & $\ldots$ & $\ldots$ \\
\hline Cuba & 647,3 & 668,7 & 643,2 & 661,5 & 672,0 & 711,7 & 696,5 & 706,8 & 733,9 & 721,0 & 759,2 & 747,4 & 768,5 & 806,4 & 800,6 & 785,9 & 790,1 & $\ldots$ \\
\hline Ecuador & 765,8 & 715,7 & 678,0 & 692,1 & 653,6 & 612,2 & 594,5 & 587,3 & 594,0 & 572,0 & 538,4 & 563,2 & 550,4 & 530,8 & 508,9 & 496,8 & 502,7 & $\ldots$ \\
\hline El Salvador & $\ldots$ & 1062,9 & 882,3 & 851,8 & 742,4 & $\ldots$ & $\ldots$ & $\ldots$ & $\ldots$ & $\ldots$ & 667,9 & 633,5 & 641,6 & 648,5 & $\ldots$ & $\ldots$ & $\ldots$ & $\ldots$ \\
\hline México & 647,9 & 704,8 & 671,0 & 646,8 & 624,8 & 614,6 & 579,9 & 578,5 & 580,2 & 579,5 & 566,2 & 541,8 & 531,5 & 525,2 & 535,3 & 536,5 & $\ldots$ & $\ldots$ \\
\hline Panamá & 461,5 & 452,0 & 450,6 & 459,7 & 433,6 & 467,0 & 462,5 & 458,5 & 465,3 & 480,2 & $\ldots$ & $\ldots$ & $\ldots$ & $\ldots$ & $\ldots$ & $\ldots$ & 483,9 & 517,0 \\
\hline Puerto Rico & 761,5 & 780,5 & 772,5 & 773,2 & 768,6 & 822,3 & 831,2 & 843,2 & 877,0 & 899,2 & 896,7 & 903,5 & 931,1 & $\ldots$ & $\ldots$ & $\ldots$ & $\ldots$ & $\ldots$ \\
\hline Trinidad y Tobago & 746,6 & 732,1 & 762,8 & 730,4 & 758,1 & 755,1 & 712,6 & 752,5 & 719,7 & 742,6 & 722,2 & 723,5 & 745,9 & 752,0 & 799,7 & $\ldots$ & $\ldots$ & $\ldots$ \\
\hline Estados Unidos & 969,5 & 950,0 & 934,5 & 939,3 & 934,6 & 944,3 & 941,0 & 935,8 & 941,9 & $\ldots$ & $\ldots$ & $\ldots$ & $\ldots$ & $\ldots$ & $\ldots$ & 891,4 & 876,3 & 861,6 \\
\hline Uruguay & 1150,7 & 1073,5 & 1051,5 & 1088,3 & 1135,5 & 1069,0 & 1058,8 & 1091,6 & 1130,8 & 1080,5 & 1098,1 & $\ldots$ & $\ldots$ & $\ldots$ & $\ldots$ & $\ldots$ & $\ldots$ & ... \\
\hline Venezuela & 575,9 & 582,8 & 558,9 & 535,0 & $\ldots$ & 515,7 & 499,9 & 503,1 & 497,8 & 510,0 & 523,4 & $\ldots$ & 518,2 & 522,1 & 536,3 & $\ldots$ & $\ldots$ & $\ldots$ \\
\hline
\end{tabular}

Fuente/Source: OPS. Sistema de Información Técnica

*Tasa/Rate 100.000 habitantes 
Cuadro 1.2 - Tasa* de mortalidad por todas las causas. Región de las Américas - 1980-1997. Toda la población femenina Table 1.2 - Mortality rate due to all causes. Region of the America - 1980-1997. Female population.

\begin{tabular}{|c|c|c|c|c|c|c|c|c|c|c|c|c|c|c|c|c|c|c|}
\hline País/Año & 1980 & 1981 & 1982 & 1983 & 1984 & 1985 & 1986 & 1987 & 1988 & 1989 & 1990 & 1991 & 1992 & 1993 & 1994 & 1995 & 1996 & 1997 \\
\hline Argentina & 730,0 & 714,7 & 685,3 & 732,0 & 734,0 & 687,3 & 677,0 & 689,0 & 697,4 & 678,8 & 689,7 & 671,3 & 681,0 & 681,3 & 642,5 & 672,2 & 657,7 & $\ldots$ \\
\hline Brasil & 524,9 & 509,6 & 487,7 & 493,9 & 505,8 & 481,1 & 483,3 & 468,0 & 479,1 & 453,1 & 449,5 & 432,4 & 438,7 & 460,8 & 460,3 & 458,5 & $\ldots$ & $\ldots$ \\
\hline Canadá & 599,0 & 593,1 & 605,7 & 602,1 & 603,9 & 619,5 & 629,3 & 623,7 & 630,8 & 628,6 & 628,2 & 635,0 & 631,0 & 656,9 & 662,0 & 664,9 & $\ldots$ & $\ldots$ \\
\hline Chile & 586,3 & 543,1 & 526,9 & 542,4 & 537,0 & 535,7 & 518,0 & 513,3 & 525,7 & 511,0 & 522,7 & 494,5 & 491,0 & 496,3 & 480,4 & $\ldots$ & $\ldots$ & $\ldots$ \\
\hline Colombia & $\ldots$ & 532,7 & $\ldots$ & $\ldots$ & 378,3 & 411,2 & 373,9 & 377,9 & 367,6 & 360,6 & 350,0 & 352,1 & 353,4 & 350,3 & 345,2 & 347,2 & $\ldots$ & $\ldots$ \\
\hline Costa Rica & 335,3 & 335,3 & 328,5 & 333,5 & 340,6 & 347,6 & 339,3 & 334,3 & 330,9 & 330,3 & 322,4 & 334,0 & 321,4 & 327,9 & 334,0 & 342,8 & $\ldots$ & $\ldots$ \\
\hline Cuba & 500,4 & 514,2 & 496,1 & 511,8 & 520,9 & 562,6 & 541,1 & 556,6 & 572,2 & 561,3 & 601,4 & 593,0 & 629,9 & 637,0 & 633,9 & 635,3 & 655,5 & $\ldots$ \\
\hline Ecuador & 665,9 & 625,6 & 582,2 & 585,4 & 543,7 & 511,1 & 497,3 & 490,8 & 482,1 & 459,1 & 439,6 & 451,9 & 444,1 & 424,0 & 402,7 & 390,4 & 390,7 & $\ldots$ \\
\hline El Salvador & $\cdots$ & $\cdots$ & 545,6 & 543,1 & 481,5 & $\cdots$ & $\ldots$ & $\cdots$ & $\cdots$ & $\ldots$ & 441,4 & 397,9 & 413,2 & 416,5 & $\cdots$ & $\cdots$ & $\cdots$ & $\cdots$ \\
\hline México & 552,9 & 522,3 & 493,0 & 493,0 & 478,9 & 473,5 & 446,3 & 445,2 & 437,5 & 441,0 & 432,0 & 409,0 & 397,2 & 399,3 & 401,2 & 408,4 & $\ldots$ & $\ldots$ \\
\hline Panamá & 353,4 & 346,6 & 347,1 & 356,0 & 342,2 & 361,2 & 344,3 & 346,4 & 347,4 & 331,1 & $\ldots$ & $\cdots$ & $\ldots$ & $\ldots$ & $\ldots$ & $\ldots$ & 351,2 & 379,4 \\
\hline Puerto Rico & 518,2 & 526,4 & 542,4 & 529,0 & 541,2 & 562,8 & 553,5 & 560,5 & 581,4 & 595,5 & 593,7 & 585,2 & 606,0 & $\cdots$ & $\ldots$ & $\cdots$ & $\ldots$ & $\cdots$ \\
\hline Trinidad y Tobago & 640,9 & 608,7 & 610,4 & 606,7 & 608,3 & 629,3 & 596,3 & 599,8 & 612,7 & 602,8 & 605,0 & 587,3 & 604,4 & 627,4 & 635,9 & $\ldots$ & $\ldots$ & $\ldots$ \\
\hline Estados Unidos & 782,7 & 774,8 & 771,3 & 788,7 & 794,8 & 809,0 & 812,0 & 815,9 & 829,9 & 817,1 & 808,1 & 810,0 & 805,8 & 838,1 & 836,8 & 841,1 & 842,4 & 841,8 \\
\hline Uruguay & 900,7 & 814,0 & 794,6 & 834,0 & 876,5 & 835,2 & 847,0 & 876,7 & 894,7 & 850,6 & 860,7 & $\cdots$ & $\cdots$ & $\cdots$ & $\cdots$ & $\cdots$ & $\cdots$ & $\cdots$ \\
\hline Venezuela & 440,7 & 451,3 & 423,5 & 404.2 & $\ldots$ & 409,4 & 391,0 & 393,1 & 381,7 & 381,4 & 399.3 & $\ldots$ & 376,6 & 370,0 & 388,7 & $\ldots$ & $\ldots$ & $\ldots$ \\
\hline
\end{tabular}

Fuente/Source: OPS. Sistema de Información Técnica

*Tasa/Rate $\times 100.000$ habitantes 
Cuadro 1.3 - Tasa* de mortalidad por causas externas. Región de las Américas - 1980-1997. Toda la población

Table 1.3 - Mortality rate due to external causes. Region of the America - 1980-1997. Entire population.

\begin{tabular}{|c|c|c|c|c|c|c|c|c|c|c|c|c|c|c|c|c|c|c|}
\hline País/Año & 1980 & 1981 & 1982 & 1983 & 1984 & 1985 & 1986 & 1987 & 1988 & 1989 & 1990 & 1991 & 1992 & 1993 & 1994 & 1995 & 1996 & 1997 \\
\hline Argentina & 58,4 & 58,7 & 53,7 & 54,5 & 53,9 & 49,0 & 54,2 & 53,7 & 54,6 & 54,4 & 52,2 & 52,2 & 54,0 & 55,6 & 55,2 & 51,9 & 53,0 & ... \\
\hline Brasil & 57,9 & 57,9 & 57,9 & 60,2 & 62,3 & 63,6 & 69,7 & 67,3 & 67,2 & 70,1 & 68,0 & 67,9 & 64,8 & 67,0 & 68,3 & 72,1 & $\ldots$ & $\ldots$ \\
\hline Canadá & 64,0 & 62,5 & 56,4 & 55,6 & 54,6 & 51,7 & 52,3 & 53,3 & 50,4 & 50,4 & 47,0 & 47,0 & 46,0 & 47,1 & 45,3 & 45,8 & $\ldots$ & $\ldots$ \\
\hline Chile & 78,4 & 78,1 & 73,7 & 78,8 & 77,6 & 74,6 & 71,6 & 67,3 & 71,1 & 74,9 & 72,9 & 69,1 & 65,2 & 66,3 & 63,6 & $\ldots$ & $\ldots$ & $\ldots$ \\
\hline Colombia & $\ldots$ & 94,6 & $\ldots$ & $\ldots$ & 78,9 & 92,3 & 93,5 & 97,6 & 104,4 & 105,7 & 107,9 & 121,8 & 122,1 & 120,3 & 114,5 & 107,8 & $\ldots$ & ... \\
\hline Costa Rica & 54,2 & 41,6 & 40,0 & 35,2 & 40,5 & 43,5 & 40,1 & 43,1 & 42,4 & 44,3 & 43,5 & 39,7 & 45,7 & 43,0 & 47,0 & 46,9 & $\ldots$ & $\ldots$ \\
\hline Cuba & 66,2 & 66,9 & 68,0 & 66,7 & 67,4 & 71,3 & 73,3 & 70,9 & 79,6 & 79,1 & 80,0 & 83,3 & 82,7 & 84,3 & 85,0 & 84,7 & 79,3 & $\ldots$ \\
\hline Ecuador & 72,0 & 72,7 & 72,0 & 73,1 & 68,7 & 65,7 & 65,2 & 64,5 & 66,3 & 64,5 & 64,5 & 65,6 & 66,7 & 67,9 & 63,9 & 65,1 & 65,4 & $\ldots$ \\
\hline El Salvador & $\ldots$ & 256,4 & 178,6 & 156,9 & 125,9 & $\ldots$ & $\ldots$ & $\ldots$ & $\ldots$ & $\ldots$ & 106,3 & 102,4 & $\ldots$ & $\ldots$ & $\ldots$ & $\ldots$ & $\ldots$ & $\ldots$ \\
\hline México & 102,1 & 101,2 & 96,9 & 83,2 & 83,5 & 84,3 & 80,9 & 78,1 & 74,9 & 72,4 & 69,7 & 68,7 & 68,2 & 64,7 & 65,4 & 62,4 & $\ldots$ & ... \\
\hline Panamá & 53,1 & 47,6 & 52,4 & 48,6 & 49,4 & 49,5 & 49,2 & 50,9 & 47,7 & 57,9 & $\ldots$ & $\ldots$ & $\ldots$ & $\ldots$ & $\ldots$ & $\ldots$ & 51,4 & 56,9 \\
\hline Puerto Rico & 60,5 & 59,1 & 55,5 & 54,1 & 56,9 & 63,1 & 66,5 & 63,1 & 67,0 & 64,2 & 65,5 & 70,2 & 72,1 & $\ldots$ & $\ldots$ & $\ldots$ & $\ldots$ & $\ldots$ \\
\hline Trinidad y Tobago & 58,1 & 54,4 & 65,8 & 59,9 & 59,7 & 60,3 & 57,9 & 58,3 & 52,8 & 52,0 & 52,8 & 50,8 & 51,6 & 55,0 & 51,4 & $\ldots$ & $\ldots$ & $\ldots$ \\
\hline Estados Unidos & 70,5 & 67,7 & 63,7 & 61,5 & 61,4 & 61,1 & 62,8 & 61,7 & 62,2 & 61,0 & 60,1 & 59,1 & 57,1 & 58,6 & 57,9 & 56,5 & 55,8 & 55,1 \\
\hline Uruguay & 62,8 & 61,6 & 65,4 & 58,4 & 53,0 & 50,8 & 56,3 & 60,2 & 58,0 & 64,3 & 61,8 & $\ldots$ & $\ldots$ & $\ldots$ & $\ldots$ & $\ldots$ & $\ldots$ & ... \\
\hline Venezuela & 76,9 & 74,5 & 73,9 & 71,7 & $\ldots$ & 61,6 & 60,7 & 60,5 & 59,6 & 61,0 & 61,2 & $\ldots$ & 68,6 & 71,9 & 74,1 & $\ldots$ & $\ldots$ & $\ldots$ \\
\hline
\end{tabular}

Fuente/Source: OPS. Sistema de Información Técnica

*Tasa/Rate 100.000 habitantes 
Cuadro 1.4 - Tasa* de mortalidad por causas externas. Región de las Américas - 1980-1997. Toda la población masculina

Table 1.4 - Mortality rate due to external causes. Region of the America - 1980-1997. Male population.

\begin{tabular}{|c|c|c|c|c|c|c|c|c|c|c|c|c|c|c|c|c|c|c|}
\hline País/Año & 1980 & 1981 & 1982 & 1983 & 1984 & 1985 & 1986 & 1987 & 1988 & 1989 & 1990 & 1991 & 1992 & 1993 & 1994 & 1995 & 1996 & 1997 \\
\hline Argentina & 86,0 & 86,3 & 80,1 & 79,9 & 78.9 & 70,8 & 78,8 & 76,9 & 78,8 & 79,0 & 75,2 & 75,7 & 78,8 & 81,7 & 81,9 & 77,3 & 79,7 & $\ldots$ \\
\hline Brasil & 92,2 & 92,7 & 93,1 & 97,5 & 101,8 & 103,6 & 113,3 & 110,5 & 110,2 & 116,4 & 112,9 & 112,6 & 107,9 & 110,9 & 113,2 & 119,9 & $\ldots$ & $\ldots$ \\
\hline Canadá & 91,0 & 89,7 & 80,4 & 79,2 & 77,3 & 73,0 & 73,0 & 74,8 & 71,0 & 70,0 & 65,8 & 65,6 & 64,1 & 65,1 & 62,7 & 29,0 & $\ldots$ & $\ldots$ \\
\hline Chile & 123,2 & 122,0 & 117,0 & 125,9 & 124,8 & 118,5 & 112,5 & 105,9 & 113,2 & 120,0 & 116,9 & 110,0 & 103,5 & 107,2 & 102,2 & $\ldots$ & $\ldots$ & $\ldots$ \\
\hline Colombia & 85,5 & 157,3 & $\ldots$ & $\ldots$ & 132,0 & 156,4 & 161,8 & 168,9 & 182,7 & 185,4 & 189,3 & 216,0 & 215,9 & 211,8 & 202,0 & 188,5 & $\ldots$ & $\ldots$ \\
\hline Costa Rica & 164,6 & 64,6 & 63,8 & 54,9 & 62,9 & 69,4 & 63,2 & 67,0 & 64,1 & 68,1 & 68,6 & 61,6 & 71,3 & 66,8 & 71,2 & 72,8 & $\ldots$ & ... \\
\hline Cuba & 85,0 & 86,8 & 86,9 & 86,3 & 88,9 & 93,2 & 97,0 & 97,9 & 106,4 & 106,1 & 107,9 & 107,5 & 105,3 & 109,2 & 114,0 & 109,5 & 104,2 & $\ldots$ \\
\hline Ecuador & 108,9 & 109,7 & 109,2 & 111,4 & 104,9 & 100,4 & 100,3 & 99,8 & 104,4 & 100,7 & 99,5 & 102,6 & 105,6 & 105,7 & 100,7 & 103,8 & 103,6 & $\ldots$ \\
\hline El Salvador & $\ldots$ & 453,3 & 311,1 & 277,1 & 218,5 & $\cdots$ & $\ldots$ & $\cdots$ & $\cdots$ & $\ldots$ & 181,0 & 176,9 & $\cdots$ & $\cdots$ & $\cdots$ & $\cdots$ & $\cdots$ & $\cdots$ \\
\hline México & 164,6 & 162,1 & 156,7 & 135,8 & 133,3 & 134,5 & 132,4 & 128,0 & 121,9 & 117,4 & 112,1 & 111,3 & 112,3 & 106,0 & 108,1 & 102,5 & $\ldots$ & $\ldots$ \\
\hline Panamá & 82,5 & 73,1 & 76,6 & 72,7 & 76,6 & 76,6 & 76,6 & 80,0 & 74,6 & 97,1 & $\ldots$ & $\ldots$ & $\ldots$ & $\ldots$ & $\ldots$ & ... & 80,9 & 90,7 \\
\hline Puerto Rico & 103,5 & 101,1 & 94,8 & 92,3 & 95,4 & 104,8 & 110,6 & 106,1 & 113,5 & 106,8 & 111,1 & 120,8 & 124,6 & $\ldots$ & $\ldots$ & $\ldots$ & $\ldots$ & $\ldots$ \\
\hline Trinidad y Tobago & 90,6 & 83,2 & 102,2 & 90,2 & 91,2 & 92,4 & 88,0 & 92,2 & 79,0 & 82,0 & 75,7 & 76,3 & 82,0 & 86,3 & 78,9 & $\ldots$ & $\ldots$ & $\ldots$ \\
\hline Estados Unidos & 105,0 & 100,7 & 94,7 & 90,6 & 90,3 & 89,8 & 92,6 & 90,5 & 91,1 & 89,2 & 88,6 & 86,4 & 84,0 & 85,8 & 84,8 & 81,3 & 79,4 & 78,1 \\
\hline Uruguay & 93,0 & 89,3 & 96,4 & 83,5 & 76,4 & 75,4 & 81,0 & 85,2 & 83,9 & 94,4 & 91,0 & $\cdots$ & $\cdots$ & $\cdots$ & $\cdots$ & $\cdots$ & $\cdots$ & $\cdots$ \\
\hline Venezuela & 124,3 & 118,6 & 117,9 & 115,2 & $\ldots$ & 96,9 & 96,2 & 95,6 & 94,5 & 98,1 & 96,7 & $\ldots$ & 111,6 & 118,1 & 123,2 & $\ldots$ & $\ldots$ & $\ldots$ \\
\hline
\end{tabular}

Fuente/Source: OPS. Sistema de Información Técnica

*Tasa/Rate $\times 100.000$ habitantes 
Cuadro 1.5 - Tasa* de mortalidad por causas externas. Región de las Américas - 1980-1997. Toda la población femenina

Table 1.5 - Mortality rate due to external causes. Region of the America - 1980-1997. Female population.

\begin{tabular}{|c|c|c|c|c|c|c|c|c|c|c|c|c|c|c|c|c|c|c|}
\hline País/Año & 1980 & 1981 & 1982 & 1983 & 1984 & 1985 & 1986 & 1987 & 1988 & 1989 & 1990 & 1991 & 1992 & 1993 & 1994 & 1995 & 1996 & 1997 \\
\hline Argentina & 31,5 & 31,9 & 27,8 & 29,7 & 29,4 & 26,4 & 29,1 & 29,5 & 29,5 & 29,8 & 27,9 & 28,4 & 28,8 & 29,0 & 27,5 & 25,7 & 25,7 & ... \\
\hline Brasil & 23,5 & 23,0 & 22,8 & 22,9 & 22,7 & 23,7 & 25,9 & 24,0 & 24,2 & 23,9 & 23,6 & 23,5 & 22,2 & 23,6 & 23,9 & 24,9 & $\ldots$ & $\ldots$ \\
\hline Canadá & 37,2 & 35,6 & 32,7 & 32,2 & 32,2 & 30,7 & 31,8 & 32,2 & 30,2 & 31,1 & 28,5 & 28,8 & 28,3 & 29,4 & 28,2 & 29,0 & $\ldots$ & $\ldots$ \\
\hline Chile & 34,8 & 35,3 & 31,5 & 32,9 & 31,7 & 31,8 & 31,8 & 29,6 & 30,1 & 30,9 & 29,9 & 29,3 & 27,8 & 26,3 & 25,8 & $\ldots$ & $\ldots$ & $\ldots$ \\
\hline Colombia & $\ldots$ & 32,7 & $\ldots$ & $\ldots$ & 26,4 & 29,0 & 26,1 & 27,4 & 27,3 & 27,1 & 27,8 & 29,3 & 30,1 & 30,7 & 29,0 & 28,8 & $\ldots$ & ... \\
\hline Costa Rica & 22,2 & 18,2 & 15,8 & 15,1 & 17,6 & 16,9 & 16,4 & 18,7 & 20,2 & 19,9 & 17,9 & 17,3 & 19,6 & 18,5 & 22,1 & 20,3 & $\ldots$ & $\ldots$ \\
\hline Cuba & 47,1 & 46,5 & 48,8 & 46,7 & 45,6 & 49,1 & 49,2 & 49,7 & 52,6 & 51,8 & 51,8 & 58,8 & 59,9 & 59,2 & 55,8 & 59,9 & 59,6 & $\ldots$ \\
\hline Ecuador & 34,7 & 35,3 & 34,4 & 34,4 & 32,0 & 30,5 & 29,8 & 28,8 & 27,8 & 28,0 & 29,1 & 28,2 & 27,3 & 29,8 & 26,8 & 26,1 & 26,8 & $\ldots$ \\
\hline El Salvador & $\ldots$ & 64,6 & 48,2 & 39,3 & 35,8 & $\ldots$ & $\ldots$ & $\ldots$ & $\ldots$ & $\ldots$ & 34,6 & 31,0 & $\ldots$ & $\ldots$ & $\ldots$ & $\ldots$ & $\ldots$ & $\ldots$ \\
\hline México & 37,7 & 38,3 & 34,8 & 30,4 & 30,4 & 34,1 & 29,4 & 27,4 & 27,3 & 26,8 & 26,8 & 25,9 & 24,0 & 23,4 & 23,4 & 22,9 & $\ldots$ & ... \\
\hline Panamá & 22,7 & 21,2 & 22,0 & 23,7 & 21,3 & 21,5 & 20,9 & 20,8 & 19,8 & 18,6 & $\ldots$ & $\ldots$ & $\ldots$ & $\ldots$ & $\ldots$ & $\ldots$ & 21,1 & 22,3 \\
\hline Puerto Rico & 19,8 & 19,2 & 18,3 & 18,0 & 20,4 & 23,7 & 24,9 & 22,7 & 23,3 & 24,2 & 22,7 & 22,7 & 22,9 & ... & $\ldots$ & $\ldots$ & $\ldots$ & $\ldots$ \\
\hline Trinidad y Tobago & 25,7 & 25,7 & 29,4 & 29,7 & 28,3 & 28,6 & 28,0 & 24,8 & 27,0 & 22,6 & 30,3 & 25,8 & 21,8 & 24,3 & 24,4 & $\ldots$ & $\ldots$ & ... \\
\hline Estados Unidos & 37,8 & 36,2 & 34,3 & 33,9 & 33,9 & 33,7 & 34,4 & 34,4 & 34,8 & 34,1 & 33,0 & 32,6 & 31,4 & 32,7 & 32,3 & 32,4 & 32,9 & 32,7 \\
\hline Uruguay & 33,6 & 34,5 & 35,5 & 34,3 & 30,5 & 27,3 & 32,7 & 36,4 & 33,2 & 35,6 & 33,8 & $\ldots$ & $\ldots$ & $\ldots$ & $\ldots$ & $\ldots$ & $\ldots$ & ... \\
\hline Venezuela & 28,3 & 29,4 & 29,0 & 27,3 & $\ldots$ & 25,6 & 24,6 & 24,7 & 24,0 & 23,2 & 25,1 & $\ldots$ & 24,9 & 25,0 & 24,2 & $\ldots$ & $\ldots$ & $\ldots$ \\
\hline
\end{tabular}

Fuente/Source: OPS. Sistema de Información Técnica

*Tasa/Rate 100.000 habitantes 
Cuadro 1.6 - Tasa* de mortalidad por accidentes de vehículo a motor. Región de las Américas - 1980-1997. Toda la población Table 1.6 - Mortality rate due to traffic accidents. Region of the America - 1980-1997. Entire population.

\begin{tabular}{|c|c|c|c|c|c|c|c|c|c|c|c|c|c|c|c|c|c|c|}
\hline País/Año & 1980 & 1981 & 1982 & 1983 & 1984 & 1985 & 1986 & 1987 & 1988 & 1989 & 1990 & 1991 & 1992 & 1993 & 1994 & 1995 & 1996 & 1997 \\
\hline Argentina & 13,5 & 13,1 & 11,9 & 12,0 & 11,3 & 8,8 & 10,1 & 9,9 & 9,6 & 9,7 & 9,2 & 9,5 & 10,1 & 10,9 & 12,1 & 10,9 & 10,7 & $\ldots$ \\
\hline Brasil & 16,4 & 15,4 & 16,4 & 15,5 & 16,6 & 18,0 & 21,5 & 19,7 & 19,5 & 19,8 & 19,2 & 18,6 & 17,4 & 17,6 & 18,5 & 20,4 & $\ldots$ & $\ldots$ \\
\hline Canadá & 21,9 & 21,3 & 16,2 & 16,4 & 15,5 & 15,8 & 14,9 & 15,7 & 14,7 & 15,4 & 13,1 & 12,3 & 11,5 & 11,9 & 10,5 & 10,4 & $\ldots$ & $\ldots$ \\
\hline Chile & 12,9 & 12,8 & $\ldots$ & $\ldots$ & 7,3 & 6,5 & 6,5 & 7,0 & 7,0 & 7,3 & 7,3 & 9,3 & 11,2 & 11,7 & 12,0 & $\ldots$ & $\ldots$ & $\ldots$ \\
\hline Colombia & $\ldots$ & 18,7 & $\ldots$ & $\ldots$ & 14,9 & 14,5 & 12,7 & 12,9 & 12,6 & 12,2 & 12,5 & 13,2 & 14,2 & 14,8 & 16,2 & 17,5 & $\ldots$ & $\ldots$ \\
\hline Costa Rica & 18,3 & 12,4 & 11,8 & 8,0 & 12,1 & 13,8 & 12,7 & 14,0 & 12,2 & 13,2 & 13,8 & 13,3 & 14,1 & 13,0 & 15,8 & 14,5 & $\ldots$ & $\ldots$ \\
\hline Cuba & $\ldots$ & $\cdots$ & $\cdots$ & $\cdots$ & $\cdots$ & $\cdots$ & $\cdots$ & $\cdots$ & $\cdots$ & $\cdots$ & $\ldots$ & $\ldots$ & 17,8 & 16,7 & 18,5 & 18,3 & 16,9 & $\ldots$ \\
\hline Ecuador & 26,7 & 25,2 & 22,4 & 18,2 & 20,0 & 18,7 & 18,4 & 18,1 & 20,8 & 19,0 & 20,0 & 21,0 & 19,8 & 20,3 & 16,3 & 15,8 & 14,7 & $\ldots$ \\
\hline El Salvador & $\ldots$ & 18,6 & 14,5 & 15,4 & 15,1 & $\cdots$ & $\ldots$ & $\cdots$ & $\cdots$ & $\ldots$ & 21,3 & 20,6 & 20,2 & 24,4 & $\cdots$ & $\cdots$ & $\cdots$ & $\cdots$ \\
\hline México & 23,0 & 25,4 & 22,6 & 19,8 & 17,1 & 16,3 & 15,9 & 15,4 & 15,8 & 16,6 & 16,5 & 16,4 & 15,8 & 15,9 & $\ldots$ & 16,1 & 14,9 & $\ldots$ \\
\hline Panamá & 18,0 & 17,4 & 18,5 & 16,9 & 16,3 & 15,9 & 16,6 & 17,2 & 13,8 & 13,6 & $\ldots$ & $\ldots$ & $\ldots$ & $\ldots$ & $\ldots$ & $\ldots$ & 15,0 & 18,5 \\
\hline Puerto Rico & 17,3 & 15,8 & 14,9 & 16,1 & 16,8 & 17,6 & 18,2 & 19,0 & 18,2 & 16,5 & 15,5 & 16,3 & 17,1 & $\ldots$ & $\ldots$ & $\cdots$ & $\ldots$ & $\ldots$ \\
\hline Trinidad y Tobago & 18,5 & 14,5 & 21,2 & 16,8 & 18,7 & 18,4 & 16,5 & 14,8 & 10,3 & 10,6 & 10,5 & 11,8 & 10,2 & 12,3 & 10,2 & $\ldots$ & $\ldots$ & $\ldots$ \\
\hline Estados Unidos & 22,8 & 21,8 & 19,3 & 18,5 & 19,1 & 18,8 & 19,5 & 19,5 & 19,6 & 18,8 & 18,3 & 16,9 & 15,7 & 15,9 & 15,9 & 15,9 & 15,8 & 15,6 \\
\hline Uruguay & 12,2 & 12,8 & 10,9 & 10,0 & 9,0 & 8,3 & 9,5 & 9,5 & 12,4 & 15,5 & 12,2 & $\ldots$ & $\ldots$ & $\ldots$ & $\ldots$ & $\ldots$ & $\ldots$ & $\ldots$ \\
\hline Venezuela & 34,5 & 32,4 & 31,8 & 29,4 & $\ldots$ & 23,0 & 24,7 & 24,2 & 23,2 & 20,5 & 20,7 & $\ldots$ & 23,4 & 22,9 & 22,3 & $\ldots$ & $\ldots$ & $\ldots$ \\
\hline
\end{tabular}

Fuente/Source:OPS. Sistema de Información Técnica

*Tasa/Rate x 100.000 habitantes 
Cuadro 1.7 - Tasa* de mortalidad por accidentes de vehículo a motor. Región de las Américas - 1980-1997. Toda la población masculina

Table 1.7-Mortality rate due to traffic accidents. Region of the America - 1980-1997. Male population.

\begin{tabular}{|c|c|c|c|c|c|c|c|c|c|c|c|c|c|c|c|c|c|c|}
\hline País/Año & 1980 & 1981 & 1982 & 1983 & 1984 & 1985 & 1986 & 1987 & 1988 & 1989 & 1990 & 1991 & 1992 & 1993 & 1994 & 1995 & 1996 & 1997 \\
\hline Argentina & 20,4 & 19,9 & 18,2 & 18,5 & 17,3 & 13,4 & 15,3 & 14,8 & 14,4 & 14,6 & 13,9 & 14,3 & 15,4 & 16,9 & 18,2 & 16,5 & 16,5 & ... \\
\hline Brasil & 25,3 & 24,1 & 25,5 & 24,2 & 26,0 & 28,0 & 33,4 & 30,7 & 30,4 & 30,9 & 30,2 & 29,0 & 27,5 & 27,6 & 28,8 & 32,2 & $\ldots$ & $\ldots$ \\
\hline Canadá & 31,7 & 31,8 & 23,5 & 23,9 & 22,4 & 22,3 & 21,1 & 22,5 & 21,3 & 21,4 & 18,5 & 17,1 & 15,9 & 16,2 & 14,7 & 14,2 & $\ldots$ & $\ldots$ \\
\hline Chile & 21,4 & 20,5 & $\ldots$ & $\ldots$ & 12,3 & 10,9 & 10,7 & 11,6 & 11,6 & 12,3 & 12,1 & 15,1 & 17,9 & 19,3 & 19,7 & $\ldots$ & $\ldots$ & $\ldots$ \\
\hline Colombia & $\ldots$ & 29,2 & $\ldots$ & $\ldots$ & 23,5 & 22,4 & 19,9 & 20,2 & 19,9 & 18,7 & 19,6 & 20,6 & 22,4 & 23.5 & 26,0 & 27,9 & $\ldots$ & ... \\
\hline Costa Rica & 29,3 & 20,3 & 19,3 & 12,4 & 18,7 & 22,8 & 20,1 & 22,4 & 19,0 & 21,1 & 22,3 & 21,3 & 23,3 & 21,4 & 25,6 & 23,7 & $\ldots$ & $\ldots$ \\
\hline Cuba & $\ldots$ & $\ldots$ & $\ldots$ & $\ldots$ & $\ldots$ & $\ldots$ & $\ldots$ & $\ldots$ & $\ldots$ & $\ldots$ & $\ldots$ & $\ldots$ & 28,1 & 26.4 & 29,7 & 28,2 & 26,6 & $\ldots$ \\
\hline Ecuador & 40,4 & 38,8 & 34,9 & 27,2 & 30,0 & 28,6 & 28,2 & 27,5 & 32,5 & 29,8 & 30,4 & 33,1 & 30,9 & 31,1 & 24,8 & 24,5 & 22,9 & $\ldots$ \\
\hline El Salvador & $\ldots$ & 27,9 & 23,3 & 23,8 & 23,0 & $\ldots$ & $\ldots$ & ... & $\ldots$ & $\ldots$ & 34,2 & 33,4 & 32,9 & 38,5 & $\ldots$ & $\ldots$ & $\ldots$ & ... \\
\hline México & 35,8 & 38,9 & 22,6 & 31,2 & 26,3 & 26,1 & 25,3 & 24,6 & 25,0 & 26,1 & 26,1 & 25,7 & 25,3 & 25,2 & $\ldots$ & 25,9 & 23,7 & ... \\
\hline Panamá & 26,8 & 27,0 & 28,1 & 25,9 & 25,4 & 25,9 & 26,2 & 27,9 & 20,5 & 22,0 & $\ldots$ & $\ldots$ & $\ldots$ & $\ldots$ & $\ldots$ & $\ldots$ & 23,7 & 29,4 \\
\hline Puerto Rico & 27,6 & 26,2 & 14,9 & 26,2 & 27,3 & 29,1 & 30,0 & 30,7 & 30,5 & 27,1 & 25,4 & 27,8 & 27,9 & ... & $\ldots$ & $\ldots$ & $\ldots$ & $\ldots$ \\
\hline Trinidad y Tobago & 30,7 & 23,7 & 33,8 & 25,2 & 30,0 & 29,8 & 26,3 & 26,5 & 16,9 & 18,2 & 14,8 & 16,6 & 15,5 & 19,4 & 15,8 & $\ldots$ & $\ldots$ & ... \\
\hline Estados Unidos & 34,2 & 32,7 & 28,6 & 27,3 & 27,9 & 27,2 & 28,4 & 28,0 & 28,0 & 26,5 & 26,0 & 23,8 & 21,9 & 22,1 & 21,9 & 21,6 & 21,3 & 20,8 \\
\hline Uruguay & 19,8 & 19,8 & 16,8 & 14,7 & 13,2 & 13,0 & 13,9 & 15,1 & 18,3 & 23,5 & 17,6 & ... & $\ldots$ & $\ldots$ & ... & $\ldots$ & $\ldots$ & ... \\
\hline Venezuela & 55,8 & 52,3 & 51,3 & 47,4 & $\ldots$ & 36,5 & 39,2 & 38,6 & 36,5 & 32,6 & 32,3 & $\ldots$ & 37,0 & 36,1 & 35,6 & $\ldots$ & $\ldots$ & $\ldots$ \\
\hline
\end{tabular}

Fuente/Source: OPS. Sistema de Información Técnica

*Tasa/Rate 100.000 habitantes 
Cuadro 1.8 - Tasa* de mortalidad por accidentes de vehículo a motor. Región de las Américas - 1980-1997. Toda la población femenina

Table 1.8 - Mortality rate due to traffic accidents. Region of the America - 1980-1997. Female population.

\begin{tabular}{|c|c|c|c|c|c|c|c|c|c|c|c|c|c|c|c|c|c|c|}
\hline País/Año & 1980 & 1981 & 1982 & 1983 & 1984 & 1985 & 1986 & 1987 & 1988 & 1989 & 1990 & 1991 & 1992 & 1993 & 1994 & 1995 & 1996 & 1997 \\
\hline Argentina & 6,7 & 6,5 & 5,8 & 5,7 & 5,5 & 4,1 & 4,9 & 5,0 & 4,7 & 4,8 & 4,4 & 4,7 & 4,8 & 4,9 & 5,9 & 5,1 & 4,7 & ... \\
\hline Brasil & 7,4 & 6,8 & 7,3 & 6,7 & 7,2 & 8,0 & 9,5 & 8,6 & 8,6 & 8,6 & 8,4 & 8,2 & 7,5 & 7,8 & 8,2 & 8,8 & $\ldots$ & $\ldots$ \\
\hline Canadá & 12,3 & 10,9 & 9,0 & 9,0 & 8,6 & 9,4 & 8,8 & 9,0 & 8,2 & 9,4 & 7,9 & 7,6 & 7,2 & 7,6 & 6,3 & 6,6 & $\ldots$ & ... \\
\hline Chile & 4,5 & 5,2 & $\ldots$ & $\ldots$ & 2,5 & 2,1 & 2,3 & 2,6 & 2,5 & 2,4 & 2,6 & 3,6 & 4,7 & 4,3 & 4,5 & $\ldots$ & $\ldots$ & $\ldots$ \\
\hline Colombia & $\ldots$ & 8,3 & $\ldots$ & $\ldots$ & 6,4 & 6,6 & 5,7 & 5,6 & 5,4 & 5,8 & 5,6 & 6,0 & 6,1 & 6,2 & 6,7 & 7,2 & $\ldots$ & $\ldots$ \\
\hline Costa Rica & 7,2 & 4,4 & 4,1 & 3,6 & 5,4 & 4,6 & 5,1 & 5,4 & 5,3 & 5,1 & 5,1 & 5,2 & 4,7 & 4,3 & 5,8 & 5,0 & $\ldots$ & $\ldots$ \\
\hline Cuba & $\ldots$ & $\ldots$ & $\ldots$ & $\ldots$ & $\ldots$ & $\ldots$ & $\ldots$ & $\ldots$ & $\ldots$ & $\ldots$ & $\ldots$ & $\ldots$ & 7,5 & 6,9 & 7,2 & 8,4 & 7,1 & $\ldots$ \\
\hline Ecuador & 12,8 & 11,5 & 9,8 & 9,1 & 9,8 & 8,7 & 8,6 & 8,5 & 9,0 & 8,2 & 9,4 & 8,8 & 8,6 & 9,3 & 7,7 & 7,0 & 6,5 & ... \\
\hline El Salvador & $\ldots$ & 9,6 & 5,9 & 7,2 & 7,4 & $\ldots$ & $\ldots$ & $\ldots$ & $\ldots$ & $\ldots$ & 8,9 & 8,3 & 7,9 & 10,8 & $\ldots$ & $\ldots$ & $\ldots$ & $\ldots$ \\
\hline México & 9,7 & 11,2 & 9,5 & 8,3 & 7,0 & 6,5 & 6,5 & 6,1 & 6,4 & 7,0 & 6,9 & 7,0 & 6,3 & 6,7 & $\ldots$ & 6,5 & 6,1 & ... \\
\hline Panamá & 8,9 & 7,4 & 8,6 & 7,6 & 6,9 & 5,5 & 6,8 & 6,1 & 6,8 & 5,0 & $\ldots$ & $\ldots$ & $\ldots$ & $\ldots$ & $\ldots$ & $\ldots$ & 6,0 & 7,4 \\
\hline Puerto Rico & 7,5 & 5,9 & 5,4 & 6,7 & 6,8 & 6,8 & 7,0 & 8,0 & 6,6 & 6,5 & 6,3 & 5,5 & 7,0 & $\ldots$ & $\ldots$ & $\ldots$ & $\ldots$ & $\ldots$ \\
\hline Trinidad y Tobago & 6,3 & 5,3 & 8,6 & 8,5 & 7,5 & 7,0 & 6,8 & 3,2 & 3,8 & 3,2 & 6,3 & 7,1 & 5,0 & 5,3 & 4,8 & $\ldots$ & $\ldots$ & $\ldots$ \\
\hline Estados Unidos & 12,0 & 11,5 & 10,4 & 10,2 & 10,8 & 10,8 & 11,0 & 11,4 & 11,6 & 11,5 & 11,1 & 10,3 & 9,8 & 9,9 & 10,2 & 10,3 & 10,4 & 10,5 \\
\hline Uruguay & 4,8 & 6,0 & 5,2 & 5,4 & 5,0 & 3,8 & 5,2 & 4,2 & 6,7 & 7,9 & 6,9 & $\cdots$ & $\cdots$ & $\cdots$ & $\cdots$ & $\cdots$ & $\cdots$ & $\cdots$ \\
\hline Venezuela & 12,7 & 12,1 & 11,9 & 11,1 & $\ldots$ & 9,3 & 9,9 & 9,6 & 9,6 & 8,3 & 8,9 & $\ldots$ & 9,6 & 9,5 & 8,9 & $\ldots$ & $\ldots$ & $\ldots$ \\
\hline
\end{tabular}

Fuente/Source:OPS.Sistema de Información Técnica

*Tasa/Rate 100.000 habitantes 
Cuadro 1.9 - Tasa* de mortalidad por suicidio. Región de las Américas - 1980-1997. Toda la población

Table 1.9 - Mortality rate due to suicide. Region of the America - 1980-1997. Entire population.

\begin{tabular}{|c|c|c|c|c|c|c|c|c|c|c|c|c|c|c|c|c|c|c|}
\hline País/Año & 1980 & 1981 & 1982 & 1983 & 1984 & 1985 & 1986 & 1987 & 1988 & 1989 & 1990 & 1991 & 1992 & 1993 & 1994 & 1995 & 1996 & 1997 \\
\hline Argentina & 7,1 & 7,8 & 7,0 & 6,4 & 6,3 & 6,8 & 7,5 & 7,5 & 7,6 & 7,1 & 6,7 & 6,0 & 6,7 & 6,7 & 6,7 & 6,4 & 6,4 & ... \\
\hline Brasil & 3,2 & 3,3 & 3,1 & 3,5 & 3,4 & 3,2 & 3,1 & 3,3 & 3,1 & 3,1 & 3,3 & 3,5 & 3,4 & 3,6 & 3,8 & 4,1 & $\ldots$ & $\ldots$ \\
\hline Canadá & 13,7 & 13,7 & 14,0 & 14,8 & 13,4 & 12,6 & 14,0 & 13,5 & 13,0 & 12,7 & 12,2 & 12,8 & 13,0 & 13,2 & 12,9 & 13,4 & $\ldots$ & $\ldots$ \\
\hline Chile & 4,9 & 5,8 & $\ldots$ & $\ldots$ & 6,2 & 5,7 & 5,3 & 5,5 & 5,5 & 5,6 & 5,6 & 5,9 & 4,8 & 5,4 & 5,7 & $\ldots$ & $\ldots$ & $\ldots$ \\
\hline Colombia & $\ldots$ & 3,4 & $\ldots$ & $\ldots$ & 3,5 & 3,2 & 3,0 & 2,9 & 2,8 & 2,5 & 2,5 & 2,9 & 3,0 & 3,0 & 3,2 & 3,0 & $\ldots$ & $\ldots$ \\
\hline Costa Rica & 5,3 & 4,1 & 3,7 & 5,3 & 4,4 & 4,9 & 4,8 & 4,3 & 5,0 & 5,8 & 5,2 & 4,2 & 4,7 & 4,9 & 4,7 & 5,9 & $\ldots$ & $\ldots$ \\
\hline Cuba & $\ldots$ & $\ldots$ & $\ldots$ & $\ldots$ & $\ldots$ & $\ldots$ & $\ldots$ & $\ldots$ & $\ldots$ & $\ldots$ & $\ldots$ & $\ldots$ & $\ldots$ & $\ldots$ & $\ldots$ & 20,3 & 18,3 & $\ldots$ \\
\hline Ecuador & 3,0 & 3,9 & 4,4 & 4,5 & 4,4 & 4,3 & 4,1 & 4,1 & 4,8 & 4,3 & 4,4 & 4,7 & 4,7 & 5,3 & 4,6 & 4,8 & 5,1 & $\ldots$ \\
\hline El Salvador & $\ldots$ & 10,6 & 11,8 & 13,0 & 11,9 & $\ldots$ & $\ldots$ & $\ldots$ & $\ldots$ & $\cdots$ & 11,4 & 9,0 & 8,1 & 7,9 & $\ldots$ & $\ldots$ & $\ldots$ & $\ldots$ \\
\hline México & 1,5 & 1,7 & 1,7 & 1,4 & 1,3 & 2,1 & 2,2 & 2,2 & 2,1 & 2,3 & 2,3 & 2,5 & 2,5 & 2,6 & 2,9 & 3,2 & $\ldots$ & $\ldots$ \\
\hline Panamá & 2,0 & 1,9 & 1,7 & 1,9 & 1,4 & 2,6 & 3,8 & 3,8 & 3,8 & 3,0 & $\cdots$ & $\cdots$ & $\cdots$ & $\cdots$ & $\cdots$ & $\cdots$ & 4,2 & 5,3 \\
\hline Puerto Rico & 9,1 & 9,3 & 9,2 & 9,9 & 9,0 & 8,0 & 9,1 & 7,9 & 9,1 & 9,3 & 10,5 & 10,0 & 8,7 & $\ldots$ & $\ldots$ & $\ldots$ & $\ldots$ & $\ldots$ \\
\hline Trinidad y Tobago & 4,0 & 5,3 & 7,0 & 8,8 & 6,8 & 2,5 & 8,8 & 10,0 & 13,6 & 14,2 & 13,8 & 11,8 & 12,9 & 13,6 & 11,5 & $\ldots$ & $\ldots$ & $\ldots$ \\
\hline Estados Unidos & 11,8 & 12,0 & 12,2 & 12,1 & 12,4 & 12,4 & 12,8 & 12,7 & 12,4 & 12,2 & 12,4 & 12,2 & 11,9 & 12,1 & 11,9 & 11,7 & 11,5 & 11,2 \\
\hline Uruguay & 9,5 & 9,1 & 10,9 & 11,0 & 11,2 & 9,6 & 8,8 & 8,5 & 8,8 & 11,2 & 10,3 & ... & $\ldots$ & $\ldots$ & $\ldots$ & $\ldots$ & $\ldots$ & $\ldots$ \\
\hline Venezuela & 4,8 & 4,3 & 4,3 & 4,8 & $\ldots$ & 4,6 & 4,6 & 4,1 & 4,6 & 4,8 & 5,0 & $\ldots$ & 4,7 & 4,6 & 5,1 & $\ldots$ & $\ldots$ & $\ldots$ \\
\hline
\end{tabular}

Fuente/Source: OPS. Sistema de Información Técnica

*Tasa/Rate 100.000 habitantes 
Cuadro 1.10 - Tasa* de mortalidad por suicidio. Región de las Américas - 1980-1997. Toda la población masculina

Table 1.10 - Mortality rate due to suicide. Region of the America - 1980-1997. Male population.

\begin{tabular}{|c|c|c|c|c|c|c|c|c|c|c|c|c|c|c|c|c|c|c|}
\hline País/Año & 1980 & 1981 & 1982 & 1983 & 1984 & 1985 & 1986 & 1987 & 1988 & 1989 & 1990 & 1991 & 1992 & 1993 & 1994 & 1995 & 1996 & 1997 \\
\hline Argentina & 10,5 & 11,9 & 10,7 & 9,3 & 9,6 & 9,9 & 10,9 & 10,5 & 10,9 & 10,5 & 9,7 & 9,0 & 10,4 & 10,6 & 10,4 & 10,0 & 9,9 & $\ldots$ \\
\hline Brasil & 4,5 & 4,6 & 4,5 & 5,1 & 5,0 & 4,7 & 3,1 & 5,0 & 4,7 & 4,7 & 4,9 & 5,3 & 5,4 & 5,7 & 5,9 & 6,6 & $\ldots$ & $\ldots$ \\
\hline Canadá & 20,7 & 20,8 & 21,8 & 22,9 & 20,6 & 19,9 & 21,8 & 21,1 & 20,4 & 19,8 & 19,4 & 20,6 & 20,7 & 21,1 & 20,6 & 21,5 & $\ldots$ & $\ldots$ \\
\hline Chile & 8,4 & 9,6 & $\ldots$ & $\ldots$ & 10,8 & 9,6 & 8,8 & 9,2 & 9,2 & 9,8 & 9,8 & 9,9 & 8,4 & 9,2 & 10,2 & $\ldots$ & $\ldots$ & $\ldots$ \\
\hline Colombia & $\ldots$ & 5,3 & $\ldots$ & $\ldots$ & 5,5 & 5,0 & 4,7 & 4,7 & 4,5 & 3,9 & 3,9 & 4,6 & 4,8 & 4,8 & 5,1 & 4,8 & $\ldots$ & $\ldots$ \\
\hline Costa Rica & 8,4 & 6,5 & 5,8 & 9,0 & 7,2 & 8,5 & 8,6 & 6,9 & 8,5 & 9,3 & 9,2 & 7,1 & 7,9 & 8,1 & 7,7 & 9,7 & $\ldots$ & ... \\
\hline Cuba & $\cdots$ & $\cdots$ & $\cdots$ & $\cdots$ & $\cdots$ & $\cdots$ & $\ldots$ & $\cdots$ & $\cdots$ & $\ldots$ & $\ldots$ & $\ldots$ & 24,6 & 25,8 & 26,2 & 25,6 & 24,5 & $\cdots$ \\
\hline Ecuador & 3,7 & 4,7 & 5,2 & 5,7 & 5,7 & 6,1 & 5,3 & 5,4 & 6,6 & 5,3 & 5,7 & 5,8 & 6,2 & 6,4 & 6,0 & 6,4 & 6,7 & $\ldots$ \\
\hline El Salvador & $\cdots$ & 16,3 & $\ldots$ & $\ldots$ & $\ldots$ & $\ldots$ & $\cdots$ & $\ldots$ & $\ldots$ & $\ldots$ & 15,3 & 12,4 & 10,8 & 10,4 & $\ldots$ & $\ldots$ & $\ldots$ & $\cdots$ \\
\hline México & 2,3 & 2,7 & 2,6 & 2,3 & 2,1 & 3,4 & 3,8 & 3,6 & 3,6 & 3,8 & 3,9 & 4,2 & 4,3 & 4,5 & 5,0 & 5,4 & $\ldots$ & $\ldots$ \\
\hline Panamá & 3,4 & 3,1 & 2,9 & 3,0 & 2,2 & 4,0 & 6,1 & 5,7 & 5,7 & 5,1 & $\ldots$ & $\ldots$ & $\ldots$ & $\ldots$ & $\ldots$ & $\ldots$ & 7,2 & 9,0 \\
\hline Puerto Rico & 15,8 & 16,8 & 16,3 & 17,9 & 16,5 & 14,3 & 16,5 & 14,1 & 16,9 & 17,2 & 19,5 & 18,5 & 16,1 & $\ldots$ & $\ldots$ & $\ldots$ & $\ldots$ & $\ldots$ \\
\hline Trinidad y Tobago & 6,3 & 7,7 & 10,3 & 12,6 & 10,4 & 13,7 & 11,8 & 15,5 & 19,7 & 21,8 & 20,4 & 19,0 & 21,9 & 23,4 & 18,4 & $\ldots$ & $\ldots$ & $\ldots$ \\
\hline Estados Unidos & 18,5 & 18,6 & 19,1 & 19,1 & 19,7 & 19,9 & 20,6 & 20,5 & 20,2 & 20,0 & 20,3 & 20,1 & 19,6 & 19,9 & 19,8 & 19,3 & 18,8 & 18,3 \\
\hline Uruguay & 16,0 & 15,6 & 18,0 & 18,8 & 18,3 & 16,1 & 14,0 & 13,1 & 14,3 & 18,4 & 16,6 & $\cdots$ & $\cdots$ & $\cdots$ & $\cdots$ & $\cdots$ & $\cdots$ & $\cdots$ \\
\hline Venezuela & 7,6 & 6,8 & 6,6 & 7,6 & $\ldots$ & $\ldots$ & 7,5 & 6,7 & 7,5 & 7,7 & 8,0 & $\ldots$ & 7,9 & 7,8 & 8,3 & $\ldots$ & $\ldots$ & $\ldots$ \\
\hline
\end{tabular}

Fuente/Source:OPS. Sistema de Información Técnica

*Tasa/Rate x 100.000 habitantes 
Cuadro 1.11 - Tasa* de mortalidad por suicidio. Región de las Américas - 1980-1997. Toda la población femenina

Table 1.11 - Mortality rate due to suicide. Region of the America - 1980-1997. Female population.

\begin{tabular}{|c|c|c|c|c|c|c|c|c|c|c|c|c|c|c|c|c|c|c|}
\hline País/Año & 1980 & 1981 & 1982 & 1983 & 1984 & 1985 & 1986 & 1987 & 1988 & 1989 & 1990 & 1991 & 1992 & 1993 & 1994 & 1995 & 1996 & 1997 \\
\hline Argentina & 3,8 & 3,8 & 3,4 & 3,6 & 3,2 & 3,5 & 4,1 & 4,4 & 4,1 & 3,8 & 3,6 & 3,0 & 3,0 & 2,9 & 2,9 & 2,9 & 3,0 & $\ldots$ \\
\hline Brasil & 1,9 & 1,9 & 1,7 & 2,0 & 1,7 & 1,6 & 1,7 & 1,7 & 1,6 & 1,5 & 1,6 & 1,6 & 1,5 & 1,5 & 1,6 & 1,8 & $\ldots$ & $\ldots$ \\
\hline Canadá & 6,7 & 6,7 & 6,3 & 6,8 & 6,0 & 5,3 & 6,2 & 6,0 & 5,7 & 5,8 & 5,0 & 5,1 & 5,5 & 5,4 & 5,3 & 5,4 & $\ldots$ & $\ldots$ \\
\hline Chile & 1,4 & 2,0 & $\ldots$ & $\ldots$ & 1,7 & 1,8 & 2,0 & 2,0 & 1,8 & 1,5 & 1,6 & 2,0 & 1,4 & 1,7 & 1,4 & $\ldots$ & $\ldots$ & $\ldots$ \\
\hline Colombia & $\ldots$ & 1,5 & $\ldots$ & $\ldots$ & 1,4 & 1,4 & 1,4 & 1,2 & 1,0 & 1,0 & 1,1 & 1,2 & 1,3 & 1,2 & 1,4 & 1,4 & $\ldots$ & $\ldots$ \\
\hline Costa Rica & 2,2 & 1,7 & 1,6 & 1,5 & 1,5 & 1,2 & 1,0 & 1,6 & 1,3 & 2,1 & 1,2 & 1,2 & 1,4 & 1,6 & 1,7 & 2,1 & $\ldots$ & $\ldots$ \\
\hline Cuba & $\ldots$ & $\ldots$ & $\ldots$ & $\ldots$ & $\ldots$ & $\ldots$ & $\ldots$ & $\ldots$ & $\ldots$ & $\ldots$ & $\ldots$ & $\ldots$ & 18,0 & 17,8 & 15,3 & 14,9 & 12,0 & $\ldots$ \\
\hline Ecuador & 2,2 & 3,1 & 3,6 & 3,3 & 3,2 & 2,5 & 2,8 & 2,8 & 2,9 & 3,3 & 3,0 & 3,6 & 3,2 & 4,1 & 3,1 & 3,2 & 3,4 & $\ldots$ \\
\hline El Salvador & $\ldots$ & 5,0 & $\ldots$ & $\ldots$ & $\ldots$ & $\ldots$ & $\ldots$ & $\ldots$ & $\ldots$ & $\cdots$ & 7,6 & 5,7 & 5,5 & 5,5 & $\ldots$ & $\ldots$ & $\ldots$ & $\ldots$ \\
\hline México & 0,6 & 0,8 & 0,7 & 0,6 & 0,4 & 0,7 & 0,7 & 0,7 & 0,7 & 0,7 & 0,7 & 0,7 & 0,8 & 0,7 & 0,9 & 1,0 & $\ldots$ & $\ldots$ \\
\hline Panamá & 0,5 & 0,6 & 0,5 & 0,7 & 0,5 & 1,1 & 1,4 & 1,9 & 1,8 & 0,8 & $\ldots$ & $\ldots$ & $\ldots$ & $\ldots$ & $\ldots$ & $\ldots$ & 1,1 & 1,6 \\
\hline Puerto Rico & 2,8 & 2,2 & 2,4 & 2,4 & 1,9 & 2,0 & 2,2 & 2,1 & 1,7 & 1,9 & 2,1 & 2,0 & 1,9 & $\ldots$ & $\ldots$ & $\ldots$ & $\ldots$ & $\ldots$ \\
\hline Trinidad y Tobago & 1,7 & 2,9 & 3,8 & 4,9 & 3,3 & 0,9 & 5,9 & 4,5 & 7,6 & 6,7 & 7,2 & 4,8 & 4,1 & 4,0 & 4,6 & $\ldots$ & $\ldots$ & $\ldots$ \\
\hline Estados Unidos & 5,4 & 5,8 & 5,6 & 5,4 & 5,4 & 5,2 & 5,4 & 5,2 & 5,0 & 4,8 & 4,8 & 4,7 & 4,6 & 4,6 & 4,5 & 4,4 & 4,3 & 4,4 \\
\hline Uruguay & 3,2 & 2,8 & 4,1 & 3,5 & 4,3 & 3,4 & 3,7 & 4,2 & 3,6 & 4,4 & 4,2 & ... & $\ldots$ & $\ldots$ & $\ldots$ & $\ldots$ & $\ldots$ & $\ldots$ \\
\hline Venezuela & 1,8 & 1,8 & 1,9 & 2,0 & $\ldots$ & 1,8 & 1,8 & 1,5 & 1,5 & 1,8 & 2,0 & $\ldots$ & 1,4 & 1,4 & 1,9 & $\ldots$ & $\ldots$ & $\ldots$ \\
\hline
\end{tabular}

Fuente/Source: OPS. Sistema de Información Técnica

*Tasa/Rate 100.000 habitantes 
Cuadro 1.12 - Tasa* de mortalidad por homicidio. Región de las Américas - 1980-1997. Toda la población

Table 1.12 - Mortality rate due to homicide. Region of the America - 1980-1997. Entire population.

\begin{tabular}{|c|c|c|c|c|c|c|c|c|c|c|c|c|c|c|c|c|c|c|}
\hline País/Año & 1980 & 1981 & 1982 & 1983 & 1984 & 1985 & 1986 & 1987 & 1988 & 1989 & 1990 & 1991 & 1992 & 1993 & 1994 & 1995 & 1996 & 1997 \\
\hline Argentina & 3,5 & 3,8 & 4,5 & 4,5 & 5,3 & 5,8 & 6,5 & 6,1 & 6,4 & 6,6 & 5,7 & 4,7 & 5,0 & 4,9 & 4,6 & 4,3 & 4,8 & $\ldots$ \\
\hline Brasil & 11,5 & 12,3 & 12,3 & 13,4 & 14,9 & 14,6 & 14,9 & 16,5 & 16,3 & 19,7 & 21,6 & 20,5 & 18,7 & 19,8 & 20,8 & 23,3 & $\ldots$ & $\ldots$ \\
\hline Canadá & 2,0 & 2,3 & 2,4 & 2,4 & 2,3 & 2,1 & 2,0 & 2,2 & 1,8 & 2,0 & 2,0 & 2,3 & 2,1 & 1,8 & 1,7 & 1,7 & $\ldots$ & $\ldots$ \\
\hline Chile & 2,6 & 3,0 & $\ldots$ & $\ldots$ & 3,3 & 3,2 & 3,1 & 2,7 & 3,1 & 2,9 & 3,1 & 3,4 & 3,1 & 2,6 & 2,9 & $\ldots$ & $\ldots$ & $\ldots$ \\
\hline Colombia & $\ldots$ & 37,2 & $\ldots$ & $\ldots$ & 30,2 & 38,2 & 44,2 & 47,3 & 56,1 & 63,0 & 68,7 & 82,5 & 82,5 & 79,7 & 73,1 & 60,8 & $\ldots$ & $\ldots$ \\
\hline Costa Rica & 5,7 & 4,5 & 4,1 & 3,8 & 4,5 & 4,8 & 4,0 & 4,7 & 4,0 & 4,4 & 4,5 & 4,1 & 5,0 & 5,0 & 5,3 & 5,0 & $\ldots$ & $\ldots$ \\
\hline Cuba & $\ldots$ & $\ldots$ & $\ldots$ & $\ldots$ & $\ldots$ & $\ldots$ & $\ldots$ & $\ldots$ & $\ldots$ & $\ldots$ & $\ldots$ & $\ldots$ & 6,6 & 7,8 & 8,4 & 8,0 & 6,9 & $\ldots$ \\
\hline Ecuador & 6,4 & 8,1 & 8,2 & 8,7 & 9,3 & 9,0 & 10,3 & 10,5 & 10,5 & 11,2 & 10,4 & 11,4 & 12,2 & 13,2 & 11,4 & 13,6 & 14,0 & $\ldots$ \\
\hline El Salvador & $\ldots$ & $\ldots$ & $\ldots$ & $\ldots$ & $\ldots$ & $\ldots$ & $\ldots$ & $\ldots$ & $\ldots$ & $\ldots$ & $\ldots$ & 43,5 & $\ldots$ & $\ldots$ & $\ldots$ & $\ldots$ & $\ldots$ & $\ldots$ \\
\hline México & 19,9 & 18,3 & 18,9 & 17,7 & 17,9 & 19,6 & 20,5 & 19,9 & 18,8 & 18,6 & 17,2 & 17,5 & 18,8 & 17,8 & 17,7 & 17,1 & $\ldots$ & $\ldots$ \\
\hline Panamá & 2,1 & 2,4 & 2,7 & 2,4 & 2,8 & 5,5 & 5,9 & 7,5 & 7,6 & 15,4 & $\ldots$ & $\ldots$ & ... & $\ldots$ & $\ldots$ & $\ldots$ & 8,2 & 11,0 \\
\hline Puerto Rico & 15,1 & 16,7 & 15,8 & 13,1 & 14,4 & 17,2 & 21,3 & 14,7 & 16,6 & 13,3 & 16,5 & 22,5 & 23,7 & $\ldots$ & $\ldots$ & $\ldots$ & $\ldots$ & $\ldots$ \\
\hline Trinidad y Tobago & 2,1 & 3,3 & 3,1 & 5,6 & 5,9 & 9,3 & 7,7 & 10,9 & 9,0 & 9,6 & 9,4 & 8,3 & 10,7 & 10,3 & 12,6 & $\ldots$ & $\ldots$ & $\ldots$ \\
\hline Estados Unidos & 10,7 & 10,3 & 9,6 & 8,6 & 8,4 & 8,4 & 9,0 & 8,7 & 9,0 & 9,3 & 10,0 & 10,5 & 10,0 & 10,1 & 9,6 & 8,6 & 7,8 & 7,3 \\
\hline Uruguay & 2,6 & 3,2 & 3,2 & 2,3 & 2,5 & 2,8 & 3,0 & 3,2 & 3,4 & 4,6 & 4,4 & $\ldots$ & $\cdots$ & $\cdots$ & $\cdots$ & $\cdots$ & $\cdots$ & $\ldots$ \\
\hline Venezuela & 11,7 & 9,7 & 11,5 & 13,0 & $\ldots$ & 9,5 & 8,2 & 8,1 & 9.0 & 12,9 & 13,3 & $\ldots$ & 13,4 & 15,2 & 16,8 & $\ldots$ & $\ldots$ & $\ldots$ \\
\hline
\end{tabular}

Fuente/Source:OPS. Sistema de Información Técnica

*Tasa/Rate 100.000 habitantes 
Cuadro 1.13 - Tasa* de mortalidad por homicidio. Región de las Américas - 1980-1997. Toda la población masculina

Table 1.13 - Mortality rate due to homicide. Region of the America - 1980-1997. Male population.

\begin{tabular}{|c|c|c|c|c|c|c|c|c|c|c|c|c|c|c|c|c|c|c|}
\hline País/Año & 1980 & 1981 & 1982 & 1983 & 1984 & 1985 & 1986 & 1987 & 1988 & 1989 & 1990 & 1991 & 1992 & 1993 & 1994 & 1995 & 1996 & 1997 \\
\hline Argentina & $\ldots$ & $\ldots$ & 7,5 & 7,3 & 8,6 & 9,6 & 10,6 & 10,0 & 10,5 & 11,2 & 9,4 & 7,8 & 8,1 & 8,0 & 7,6 & 7,2 & 8,1 & ... \\
\hline Brasil & 20,7 & 22,1 & 22,2 & 24,3 & 27,2 & 26,6 & 27,1 & 30,2 & 29,9 & 36,3 & 40,0 & 37,5 & 34,4 & 36,4 & 38,2 & 42,8 & $\ldots$ & $\ldots$ \\
\hline Canadá & 2,8 & 2,9 & 3,3 & 3,2 & 3,2 & 2,8 & 2,6 & 2,9 & 2,5 & 2,6 & 2,6 & 2,9 & 2,9 & 2,5 & 2,3 & 2,3 & $\ldots$ & $\ldots$ \\
\hline Chile & 4,2 & 5,1 & $\ldots$ & $\ldots$ & 5,9 & 5,7 & 5,4 & 5,0 & 5,4 & 5,3 & 5,4 & 6,1 & 5,6 & 4,9 & 5,1 & $\ldots$ & $\ldots$ & $\ldots$ \\
\hline Colombia & $\ldots$ & 69,0 & $\ldots$ & $\ldots$ & 56,4 & 71,6 & 83,2 & 89,0 & 105,1 & 118,3 & 128,1 & 154,6 & 154 & 149,1 & 136,8 & 113,9 & $\ldots$ & ... \\
\hline Costa Rica & 8,4 & 7,6 & 7,3 & 6,7 & 7,3 & 7,8 & 5,9 & 7,5 & 6,9 & 6,8 & 7,1 & 6,6 & 8,3 & 8,6 & 9,1 & 8,5 & $\ldots$ & $\ldots$ \\
\hline Cuba & $\ldots$ & $\ldots$ & $\ldots$ & $\ldots$ & $\ldots$ & $\ldots$ & $\ldots$ & $\ldots$ & $\ldots$ & $\ldots$ & $\ldots$ & $\ldots$ & 9,8 & 12,1 & 13,0 & 12,1 & 10,8 & $\ldots$ \\
\hline Ecuador & 11,2 & 14,2 & 14,6 & 15,5 & 16,8 & 15,8 & 18,4 & 18,6 & 18,9 & 19,9 & 18,7 & 20,5 & 22,2 & 23,9 & 20,9 & 24,7 & 25,6 & $\ldots$ \\
\hline El Salvador & $\ldots$ & 0,0 & $\ldots$ & $\ldots$ & $\ldots$ & $\ldots$ & $\ldots$ & $\ldots$ & $\ldots$ & $\ldots$ & $\ldots$ & 83,9 & $\ldots$ & $\ldots$ & $\ldots$ & $\ldots$ & $\ldots$ & $\ldots$ \\
\hline México & 34,7 & 33,1 & 33,9 & 32,1 & 31,1 & 35,8 & 37,3 & 36,3 & 34,0 & 33,4 & 30,7 & 31,5 & 33,9 & 32,2 & 32,3 & 31,2 & $\ldots$ & ... \\
\hline Panamá & 3,3 & 4,2 & 4,6 & 3,6 & 4,4 & 9,9 & 10,1 & $\cdots$ & $\cdots$ & 26,9 & $\cdots$ & $\cdots$ & $\cdots$ & $\cdots$ & $\cdots$ & $\cdots$ & 14,9 & 19,8 \\
\hline Puerto Rico & 27,8 & 29,7 & 15,8 & 23,7 & 25,3 & 31,5 & 36,2 & 27,5 & 30,1 & 23,5 & 29,8 & 40,4 & 44,7 & $\ldots$ & $\ldots$ & $\ldots$ & $\ldots$ & $\ldots$ \\
\hline Trinidad y Tobago & 2,8 & 4,6 & 5,6 & 7,6 & 8,9 & 13,7 & 12,5 & 16,4 & 14,4 & 14,7 & 15 & 13,5 & 17,2 & 16,1 & 19,5 & $\ldots$ & $\ldots$ & ... \\
\hline Estados Unidos & 17,2 & 16,6 & 15,3 & 13,6 & 13,1 & 13,0 & 14,2 & 13,4 & 14 & 14,7 & 16,1 & 16,9 & 16,2 & 16,1 & 15,5 & 13,5 & 12,3 & 11,5 \\
\hline Uruguay & 3,6 & 4,9 & 4,8 & 3,5 & 4,2 & 5,0 & 4,7 & 4,2 & 5,4 & 7,6 & 7,0 & $\ldots$ & $\ldots$ & $\ldots$ & $\ldots$ & $\ldots$ & $\ldots$ & $\ldots$ \\
\hline Venezuela & 21,6 & 17,6 & 20,9 & 23,9 & $\ldots$ & 17,2 & 14,9 & 14,6 & 16,5 & 23,7 & 24,4 & $\ldots$ & 24,7 & 28,0 & 31,1 & $\ldots$ & $\ldots$ & $\ldots$ \\
\hline
\end{tabular}

Fuente/Source: OPS. Sistema de Información Técnica

*Tasa/Rate 100.000 habitantes 
Cuadro 1.14 - Tasa* de mortalidad por homicidio. Región de las Américas - 1980-1997. Toda la población femenina

Table 1.14 - Mortality rate due to homicide. Region of the America - 1980-1997. Female population.

\begin{tabular}{|c|c|c|c|c|c|c|c|c|c|c|c|c|c|c|c|c|c|c|}
\hline País/Año & 1980 & 1981 & 1982 & 1983 & 1984 & 1985 & 1986 & 1987 & 1988 & 1989 & 1990 & 1991 & 1992 & 1993 & 1994 & 1995 & 1996 & 1997 \\
\hline Argentina & $\ldots$ & $\ldots$ & 1,7 & 1,7 & 2 & 1,8 & 2,2 & 1,9 & 1,9 & 2,0 & 1,6 & 1,5 & 1,9 & 1,8 & 1,6 & 1,4 & 1,5 & $\ldots$ \\
\hline Brasil & 2,2 & 2,4 & 2,4 & 2,6 & 2,6 & 2,6 & 2,6 & 2,8 & 2,8 & 3,2 & 3,5 & 3,6 & 3,1 & 3,4 & 3,6 & 4,1 & $\ldots$ & $\ldots$ \\
\hline Canadá & 1,3 & 1,7 & 1,5 & 1,6 & 1,4 & 1,5 & 1,3 & 1,5 & 1,2 & 1,5 & 1,4 & 1,6 & 1,4 & 1,2 & 1,2 & 1,1 & $\ldots$ & $\ldots$ \\
\hline Chile & 0,9 & 1,0 & $\ldots$ & $\ldots$ & 0,7 & 0,8 & 0,8 & 0,6 & 0,8 & 0,6 & 0,8 & 0,7 & 0,7 & 0,4 & 0,8 & $\ldots$ & $\ldots$ & $\ldots$ \\
\hline Colombia & $\ldots$ & 5,8 & $\ldots$ & $\ldots$ & 4,3 & 5,2 & 5,7 & 6,3 & 7,7 & 8,6 & 10,3 & 11,6 & 12,3 & 11,7 & 10,7 & 8,9 & $\ldots$ & .. \\
\hline Costa Rica & 2,9 & 1,4 & 0,9 & 0,8 & 1,7 & 1,8 & 2,1 & 1,8 & 1,1 & 1,9 & 1,8 & 1,6 & 1,6 & 1,2 & 1,5 & 1,5 & $\ldots$ & $\ldots$ \\
\hline Cuba & $\ldots$ & $\ldots$ & $\ldots$ & $\ldots$ & $\ldots$ & $\ldots$ & $\ldots$ & $\ldots$ & $\ldots$ & $\ldots$ & $\ldots$ & $\ldots$ & 3,3 & 3,4 & 3,9 & 3,8 & 3,0 & $\ldots$ \\
\hline Ecuador & 1,5 & 1,9 & 1,8 & 1,7 & 1,8 & 2,1 & 2,2 & 2,2 & 2,0 & 2,4 & 2,0 & 2,2 & 2,0 & 2,3 & 1,9 & 2,4 & 2,3 & $\ldots$ \\
\hline El Salvador & $\cdots$ & $\cdots$ & $\cdots$ & $\ldots$ & $\ldots$ & $\ldots$ & $\ldots$ & $\ldots$ & $\ldots$ & $\ldots$ & $\ldots$ & 4,8 & $\ldots$ & $\ldots$ & $\ldots$ & $\ldots$ & $\ldots$ & .. \\
\hline México & 4,4 & 3,1 & 3,2 & 3,2 & 3,7 & 3,4 & 3,6 & 3,2 & 3,5 & 3,7 & 3,6 & 3,6 & 3,7 & 3,4 & 3,3 & 3,3 & $\ldots$ & $\ldots$ \\
\hline Panamá & 0,8 & 0,5 & 0,6 & 1,2 & 1,1 & 1,0 & 1,6 & 2,7 & $\ldots$ & 3,7 & $\ldots$ & $\ldots$ & $\ldots$ & $\ldots$ & $\ldots$ & $\ldots$ & 1,4 & 2,1 \\
\hline Puerto Rico & 3,0 & 4,4 & 4,3 & 3,1 & 4,1 & 3,7 & 7,2 & 2,7 & 3,9 & 3,8 & 4,0 & 5,8 & 4,0 & $\ldots$ & $\ldots$ & $\ldots$ & $\ldots$ & ... \\
\hline Trinidad y Tobago & 1,5 & 2,0 & 0,7 & 3,5 & 2,8 & 5,0 & 3,0 & 5,5 & 3,8 & 4,5 & 3,9 & 3,2 & 4,2 & 4,7 & 5,8 & $\ldots$ & $\ldots$ & ... \\
\hline Estados Unidos & 4,4 & 4,3 & 4,2 & 3,9 & 3,9 & 4,0 & 4,2 & 4,2 & 4,2 & 4,1 & 4,2 & 4,4 & 4,1 & 4,3 & 3,9 & 3,8 & 3,4 & 3,2 \\
\hline Uruguay & 1,6 & 1,6 & 1,6 & 1,2 & 0,9 & 0,7 & 1,5 & 2,1 & 1,6 & 1,8 & 2,0 & $\cdots$ & $\cdots$ & $\cdots$ & $\cdots$ & $\cdots$ & $\cdots$ & $\cdots$ \\
\hline Venezuela & 1,6 & 1,7 & 1,9 & 1,9 & 1,9 & 1,7 & 1.4 & 1,4 & 1,5 & 1,9 & 2,0 & $\ldots$ & 1,9 & 2,1 & 2,2 & $\ldots$ & $\ldots$ & $\ldots$ \\
\hline
\end{tabular}

Fuente/Source:OPS. Sistema de Información Técnica

*Tasa/Rate 100.000 habitantes 